\title{
Experimental Advanced Airborne Research Lidar (EAARL) Data Processing Manual
}

By Jamie M. Bonisteel, Amar Nayegandhi, C. Wayne Wright, John C. Brock, and David B. Nagle

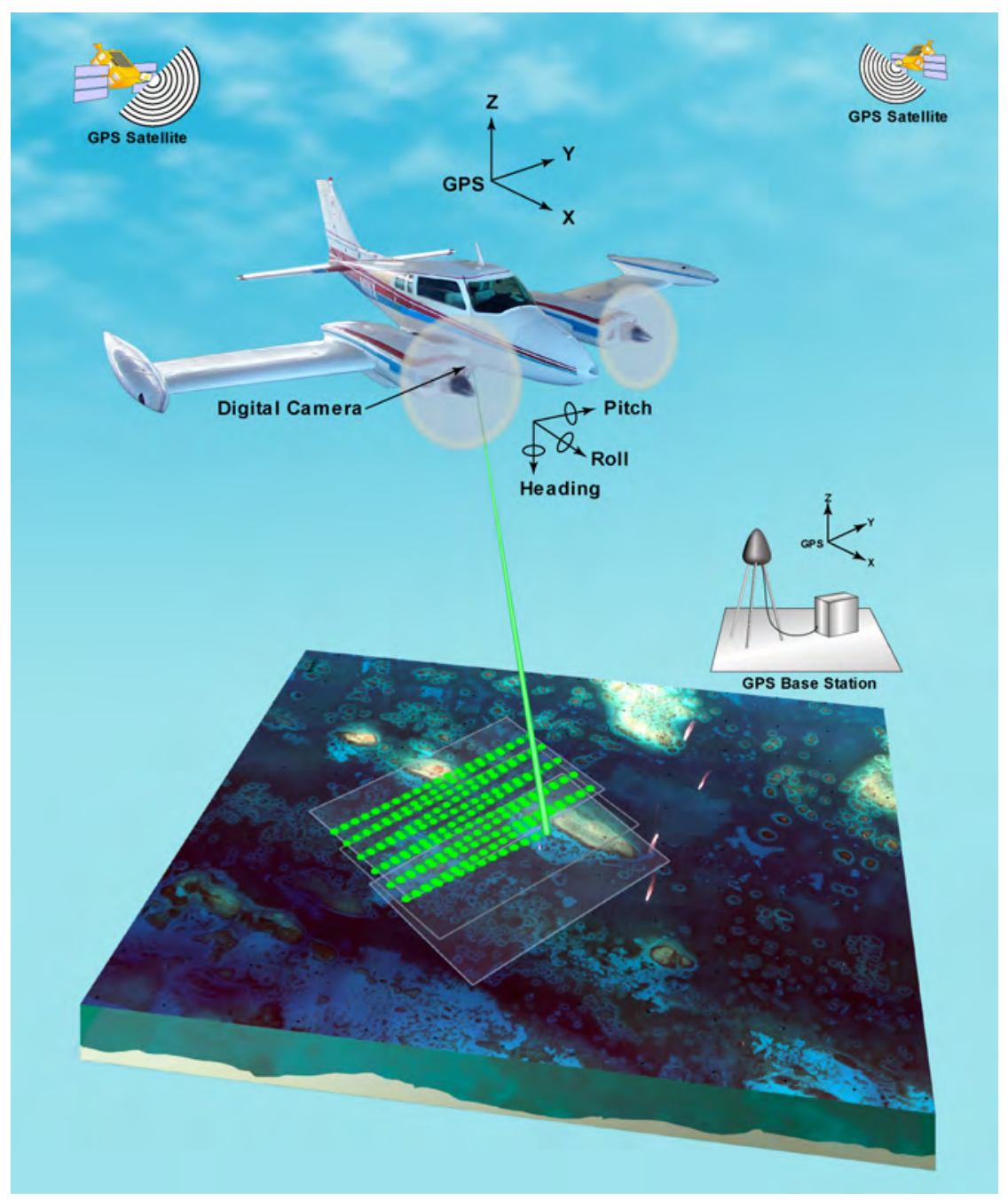

Open-File Report Series 2009-1078 


\title{
U.S. Department of the Interior KEN SALAZAR, Secretary
}

\section{U.S. Geological Survey \\ Suzette M. Kimball, Acting Director}

\author{
U.S. Geological Survey, Reston, Virginia 2009
}

For product and ordering information:

World Wide Web: http://www.usgs.gov/pubprod

Telephone: 1-888-ASK-USGS

For more information on the USGS - the Federal source for science about the Earth, its natural and living resources, natural hazards, and the environment:

World Wide Web: http://www.usgs.gov

Telephone: 1-888-ASK-USGS

Suggested citation:

Bonisteel, J.M., Nayegandhi, Amar, Wright, C.W., Brock, J. C., and Nagle, D.B., 2009, Experimental Advanced Airborne Research Lidar (EAARL) Data Processing Manual: U.S. Geological Survey Open-File Report, 2009-1078, 38p.

Any use of trade, product, or firm names is for descriptive purposes only and does not imply endorsement by the U.S. Government.

Although this report is in the public domain, permission must be secured from the individual copyright owners to reproduce any copyrighted material contained within this report. 


\section{Experimental Advanced Airborne Research Lidar (EAARL) Data Processing Manual}

By Jamie M. Bonisteel, Amar Nayegandhi, C. Wayne Wright, John C. Brock, and David B. Nagle

Open-File Report Series 2009-1078

U.S. Department of the Interior

U.S. Geological Survey 



\section{Contents}

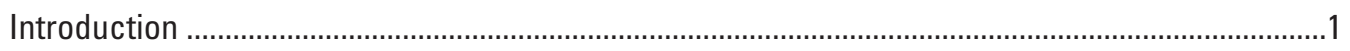

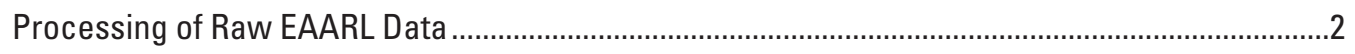

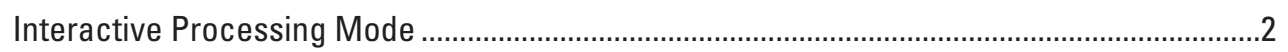

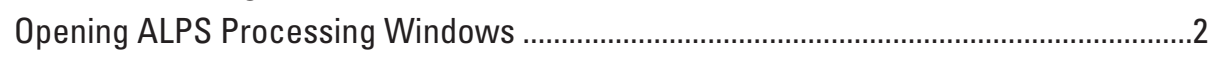

Loading the EAARL Database File ..........................................................................

Loading GPS Flight Track Information ...........................................................................

Loading Digital Miniature Attitude Reference System (DMARS) Information ...................5

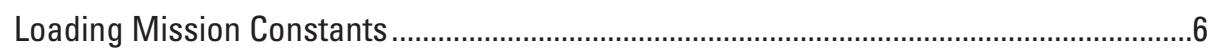

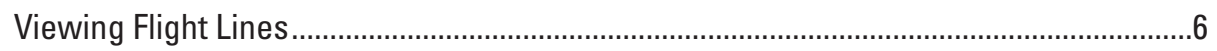

Loading Coastal Base Maps ................................................................................

Viewing Raster Images and Waveforms......................................................................

Loading Other Images ...........................................................................................

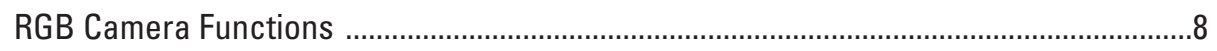

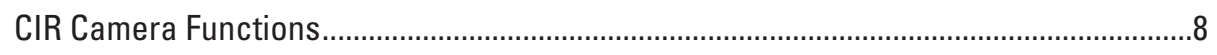

Topographic Lidar Data Processing .........................................................................

Viewing Processed Topography Data ......................................................................... 10

Submerged Topography (Bathymetry) Lidar Data Processing with Waveform Investigation ...........................................................................................11

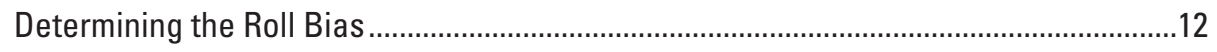

Batch Processing Mode ...............................................................................................

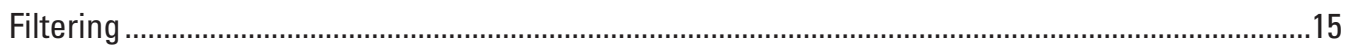

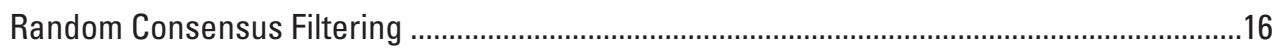

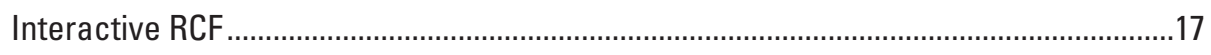

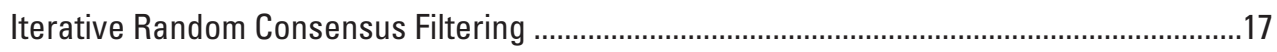

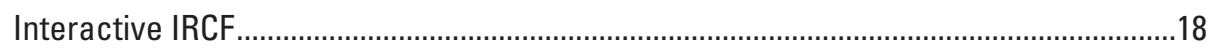

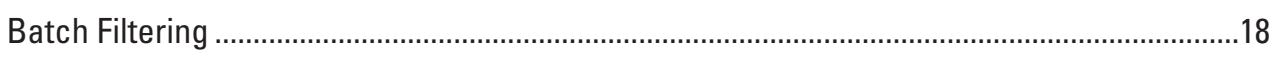

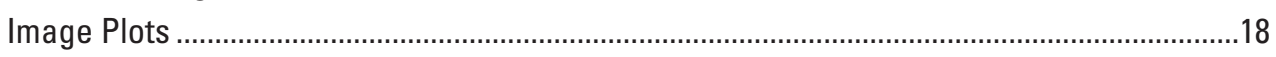

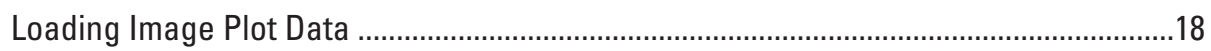

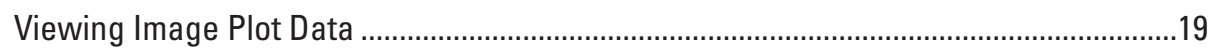

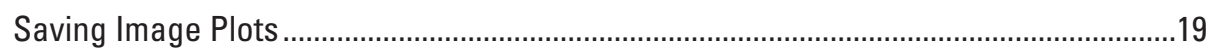

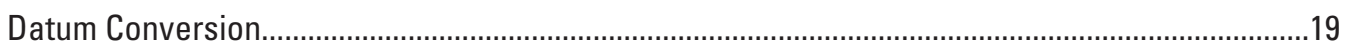

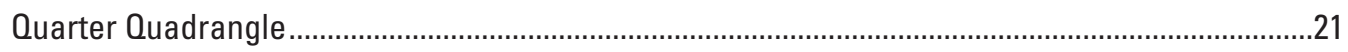

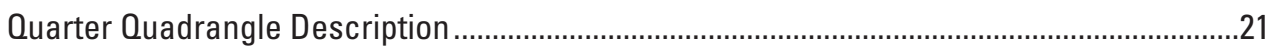

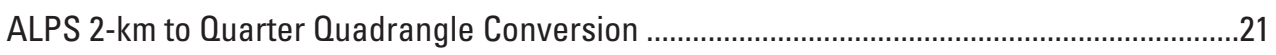

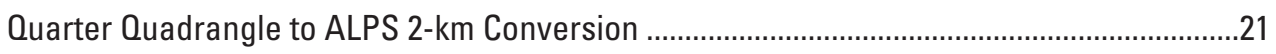

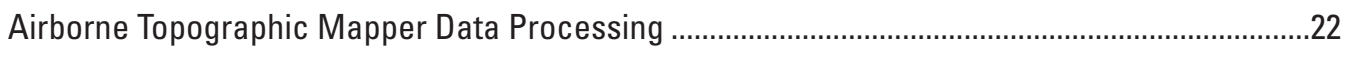

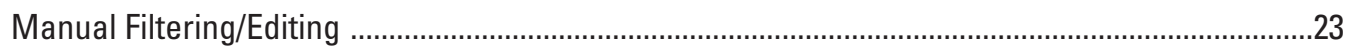

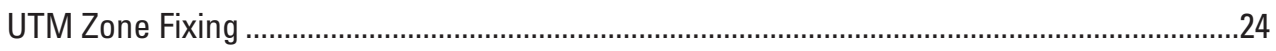

Keep/Remove/Elevation Clipper Filter Tools...................................................................24

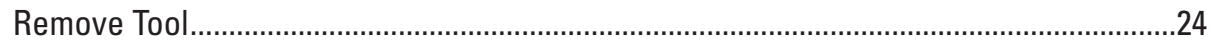




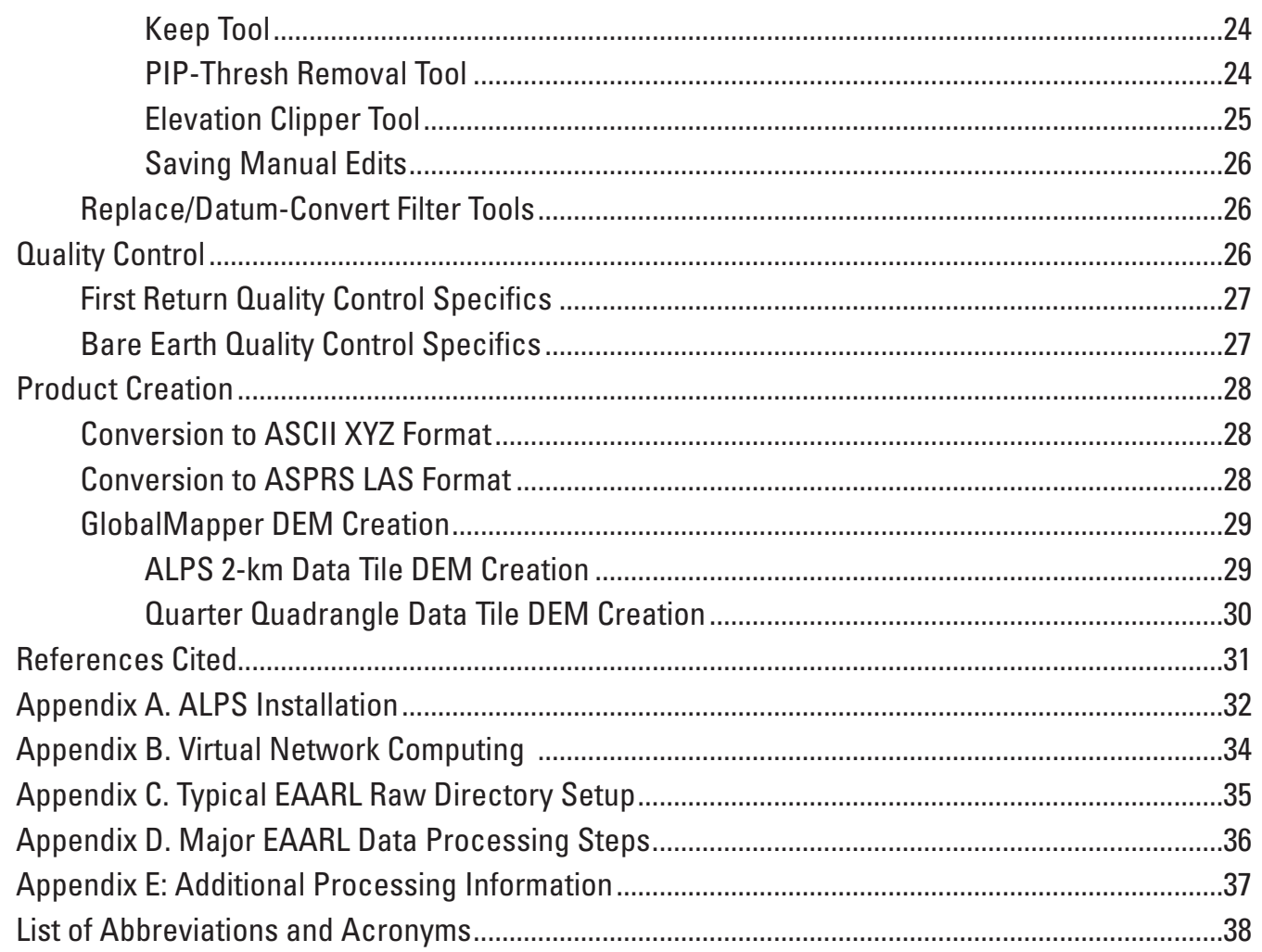




\section{Figures}

Figure 1. Sample waveform returns from vegetation and submerged topography (Wright and Brock, 2002)

Figure 2. ALPS provides an interface for data processing, visualization, and investigation............2

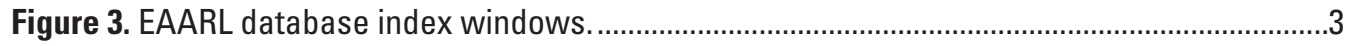

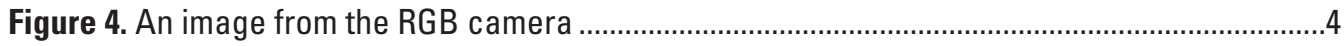

Figure 5. The Plotting Tool window manages flight lines, shapefiles, coastal base maps, and flight plans.

Figure 6. Yorick shell after the EDB, PNav, and DMARS files have been loaded ............................5

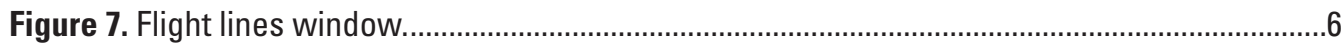

Figure 8. Screenshot from an interactive session in ALPS showing EAARL rasters (top right and left), RGB (top center) images, and flight line map (bottom left).

Figure 9. An illustration of the EAARL oscillating scan pattern and aircraft positioning (Brock and others, 2004).

7

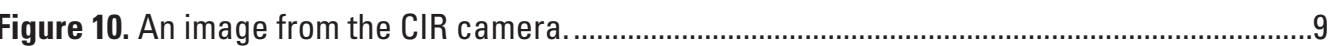

Figure 11. Rectangular coordinate (I1widc window) method. ........................................................

Figure 12. A histogram display and color bar tool window.......................................................

Figure 13. A screenshot showing processed data (top left), a georeferenced raster image (top center), a sample waveform (top right), a histogram (bottom left), the ALPS command window (bottom center), and an RGB image (bottom right....................................................11

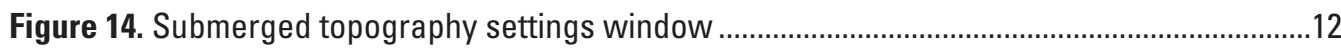

Figure 15. Submerged topography waveform display with the raw waveform (black) and the processed waveform (blue) after the submerged topography settings are applied..........13

Figure 16. The ops_conf settings window .13

Figure 17. The panel on the right shows elevation data from overlapping flight lines. A vertical transect (represented by the red line) through the data is shown on the panel on the left. Each color represents a flight line.

Figure 18. The $2-\mathrm{km}$ by $2-\mathrm{km}$ data tiles are highlighted in pink and the $10-\mathrm{km}$ by $10-\mathrm{km}$ index tiles are highlighted in purple.

Figure 19. Highlighted in green are the flight lines selected to process in batch processing mode. As the flight lines are processed, they are highlighted in black.

Figure 20. The image on the right shows a vertical slice of data from the image on the left. The image on the right shows a concentration of points on the ground and several outliers. The data outside of the red rectangle highlight the points that would be removed (Nayegandhi and others, 2004).

Figure 21. The Random Consensus Filter window.......................................................................17

Figure 22. The Iterative Random Consensus Filter window..........................................................18 
Figure 23. Image plot showing unfiltered elevation data from $-35 \mathrm{~m}$ to $-6.35 \mathrm{~m}$. Elevation values are in WGS84 ellipsoid heights. The yellow specks represent noise that filtering would help remove.

Figure 24. Image plot showing filtered elevation data from $-36.70 \mathrm{~m}$ to $-3.80 \mathrm{~m}$. Elevations values are in WGS84 ellipsoid heights. The yellow specks have mostly been filtered out............20

Figure 25. The elliptical scan pattern of the ATM system (Sallenger and Brock, 2001 ..................22

Figure 26. Keep (left) and remove (right) filter windows utilized during manual editing................24

Figure 27. Figure illustrating the use of the keep tool to retain data within the circled areas. Data outside of these areas will be removed. ............................................................................25

Figure 28. A point-cloud elevation histogram shows a well-defined peak near $0 \mathrm{~m}$. Possible outliers located to the left and right of the peak are circled in red...................................25

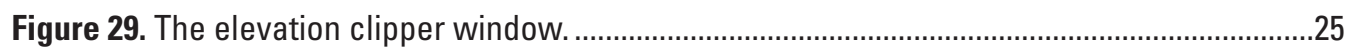

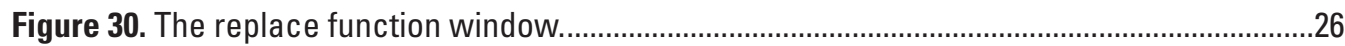

Figure 31. A considerable amount of data can be created along the edge of the swath (Nayegandhi and others, 2006)

Figure 32. An error in the data can be seen within each square of this bare earth 2-km by 2-km tile. The GPS offset occurred between the 2 days of data collection.................................27

Figure 33. Window that shows DEM script created for GlobalMapper...........................................30

Figure 34. Window that shows GlobalMapper geotiff conversion script.........................................30

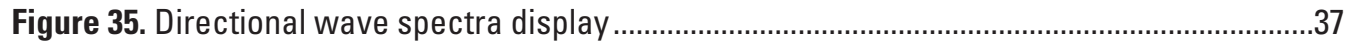

\section{Tables}

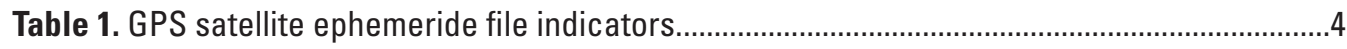

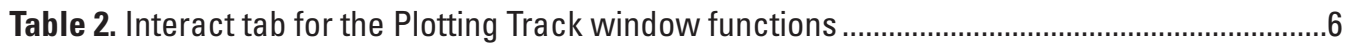

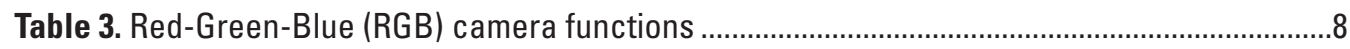

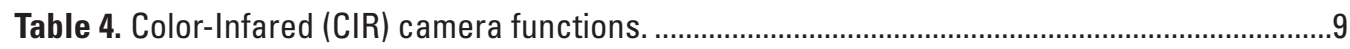

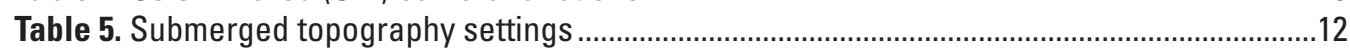

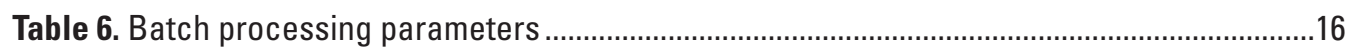

Table 7. Batch filtering parameters …………………...........................................................

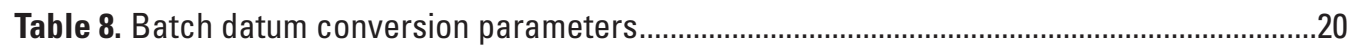

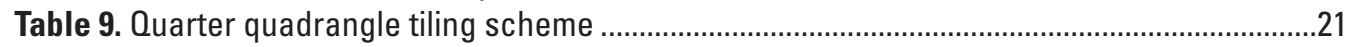

Table 10. Quarter Quadrangle to ALPS 2-km conversion parameters ..........................................22

Table 11. Quarter Quadrangle to ALPS 2-km conversion parameters ...........................................22

Table 12. Airborne Topographic Mapper processing parameters ..................................................

Table 13. Airborne Topographic Mapper merging parameters......................................................23

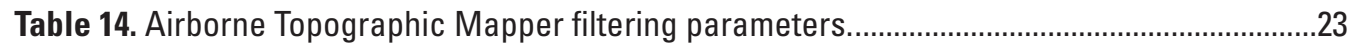

Table 15. Fix Universal Transverse Mercator or Quarter Quadrangle zones...................................24

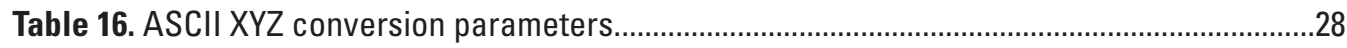

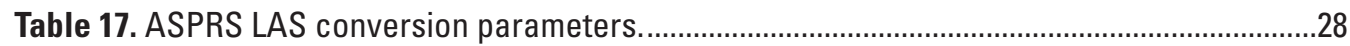

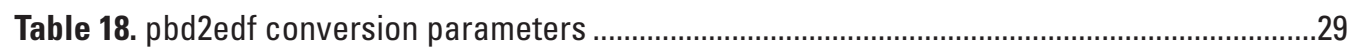

Table 19. Interactive Data Language DEM creation parameters ................................................29

Table 20. Quarter Quadrangle geotiff preparation parameters...........................................................30 


\title{
Experimental Advanced Airborne Research Lidar (EAARL) Data Processing Manual
}

\author{
By Jamie M. Bonisteel, Amar Nayegandhi, C. Wayne Wright, John C. Brock, and David Nagle
}

\section{Introduction}

The Experimental Advanced Airborne Research Lidar (EAARL) is an example of a Light Detection and Ranging (Lidar) system that utilizes a blue-green wavelength (532 nanometers) to determine the distance to an object. The distance is determined by recording the travel time of a transmitted pulse at the speed of light (fig. 1). This system uses raster laser scanning with full-waveform (multi-peak) resolving capabilities to measure submerged topography and adjacent coastal land elevations simultaneously (Nayegandhi and others, 2009).

This document reviews procedures for the postprocessing of EAARL data using the custom-built Airborne Lidar Processing System (ALPS). ALPS software was developed in an open-source programming environment operated on a Linux platform. It has the ability to combine the laser return backscatter digitized at 1-nanosecond intervals

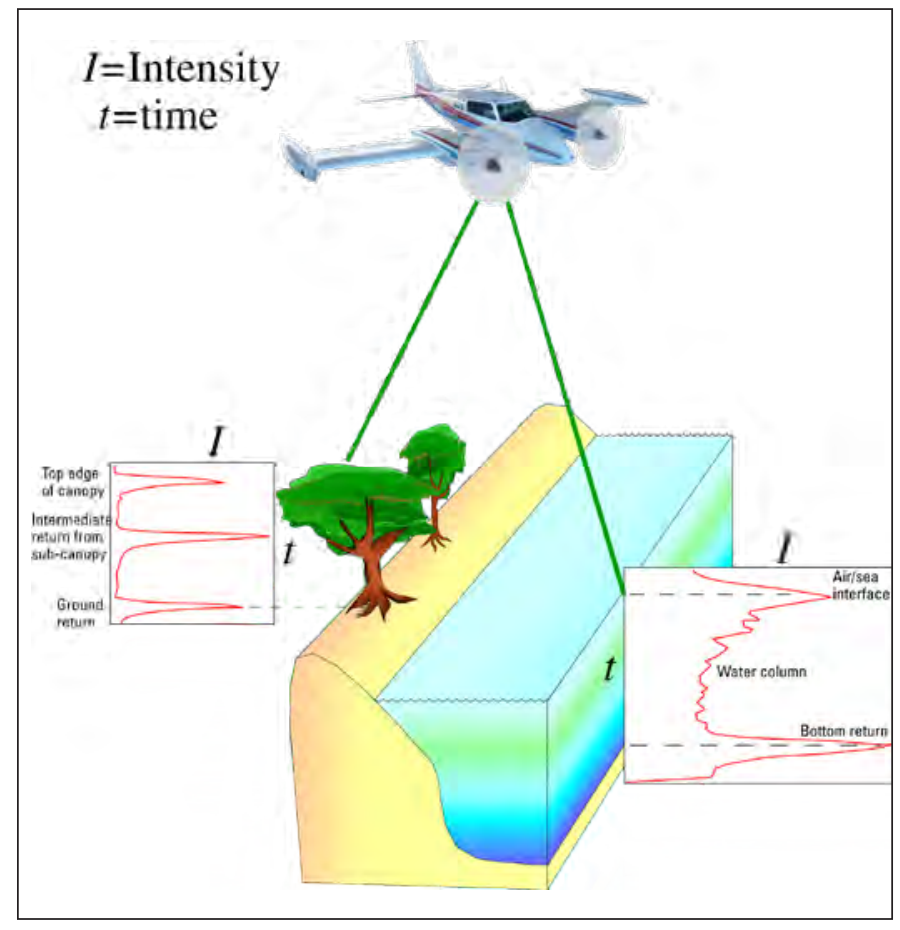

Figure 1. Sample waveform returns from vegetation and submerged topography (Wright and Brock, 2002). with aircraft positioning information. This solution enables the exploration and processing of the EAARL data in an interactive or batch mode. ALPS also includes modules for the creation of bare earth, canopy-top, and submerged topography Digital Elevation Models (DEMs). The EAARL system uses an Earth-centered coordinate and reference system that removes the necessity to reference submerged topography data relative to water level or tide gages (Nayegandhi and others, 2006).

The EAARL system can be mounted in an array of small twin-engine aircraft that operate at 300 meters above ground level (AGL) at a speed of 60 meters per second (117 knots). While other systems strive to maximize operational depth limits, EAARL has a narrow transmit beam and receiver field of view (1.5 to 2 milliradians), which improves the depthmeasurement accuracy in shallow, clear water but limits the maximum depth to about 1.5 Secchi disk depth ( 20 meters) in clear water. The laser transmitter [Continuum EPO-5000 yttrium aluminum garnet (YAG)] produces up to 5,000 shortduration (1.2 nanosecond), low-power (70 microjoules) pulses each second. Each pulse is focused into an illumination area that has a radius of about 20 centimeters on the ground. The pulse-repetition frequency of the EAARL transmitter varies along each across-track scan to produce equal cross-track sample spacing and near uniform density (Nayegandhi and others, 2006).

Targets can have varying physical and optical characteristics that cause extreme fluctuations in laser backscatter complexity and signal strength. To accommodate this dynamic range, EAARL has the real-time ability to detect, capture, and automatically adapt to each laser return backscatter. The backscattered energy is collected by an array of four high-speed waveform digitizers connected to an array of four sub-nanosecond photodetectors. Each of the four photodetectors receives a finite range of the returning laser backscatter photons. The most sensitive channel receives $90 \%$ of the photons, the least sensitive receives $0.9 \%$, and the middle channel receives 9\% (Wright and Brock, 2002). The fourth channel is available for detection but is not currently being utilized. All four channels are digitized simultaneously into 65,536 samples for every laser pulse. Receiver optics consists of a 15-centimeter-diameter dielectric-coated Newtonian telescope, a computer-driven raster scanning mirror oscillating at 12.5 hertz ( 25 rasters per second), and an array of sub-nanosecond photodetectors. The signal emitted 
by the pulsed laser transmitter is amplified as backscatter by the optical telescope receiver. The photomultiplier tube (PMT) then converts the optical energy into electrical impulses (Nayegandhi and others, 2006).

In addition to the full-waveform resolving laser, the EAARL sensor suite includes a down-looking 70-centimeterresolution Red-Green-Blue (RGB) digital network camera, a high-resolution color infrared (CIR) multispectral camera (14-centimeter-resolution), two precision dual-frequency kinematic carrier-phase global positioning system (GPS) receivers, and an integrated gyroscope-based, miniature digital inertial measurement unit (IMU) that provides accurate attitude information (Nayegandhi and others, 2006).

Laser error depends on the type of surface being mapped, which can range from a few centimeters in open canopies to several meters in closed and sloping terrains. Recent studies have shown that EAARL elevation root mean square errors (RMSE) range from 10 to 14 centimeters for submerged topography to 16 to 20 centimeters for sub-canopy topography. The RMSE is also highly dependent on GPS satellite configuration and lidar sampling angles. A high RMSE occurs during poor satellite configuration and large sampling angles that usually happen at the edge of the swath (Nayegandhi and others, 2009).

\section{Processing of Raw EAARL Data}

For waveform-resolving instruments such as EAARL, the range is determined in post-processing. Processing algorithms have been developed to extract the range to the first and last significant return. The shape of the waveform is determined by

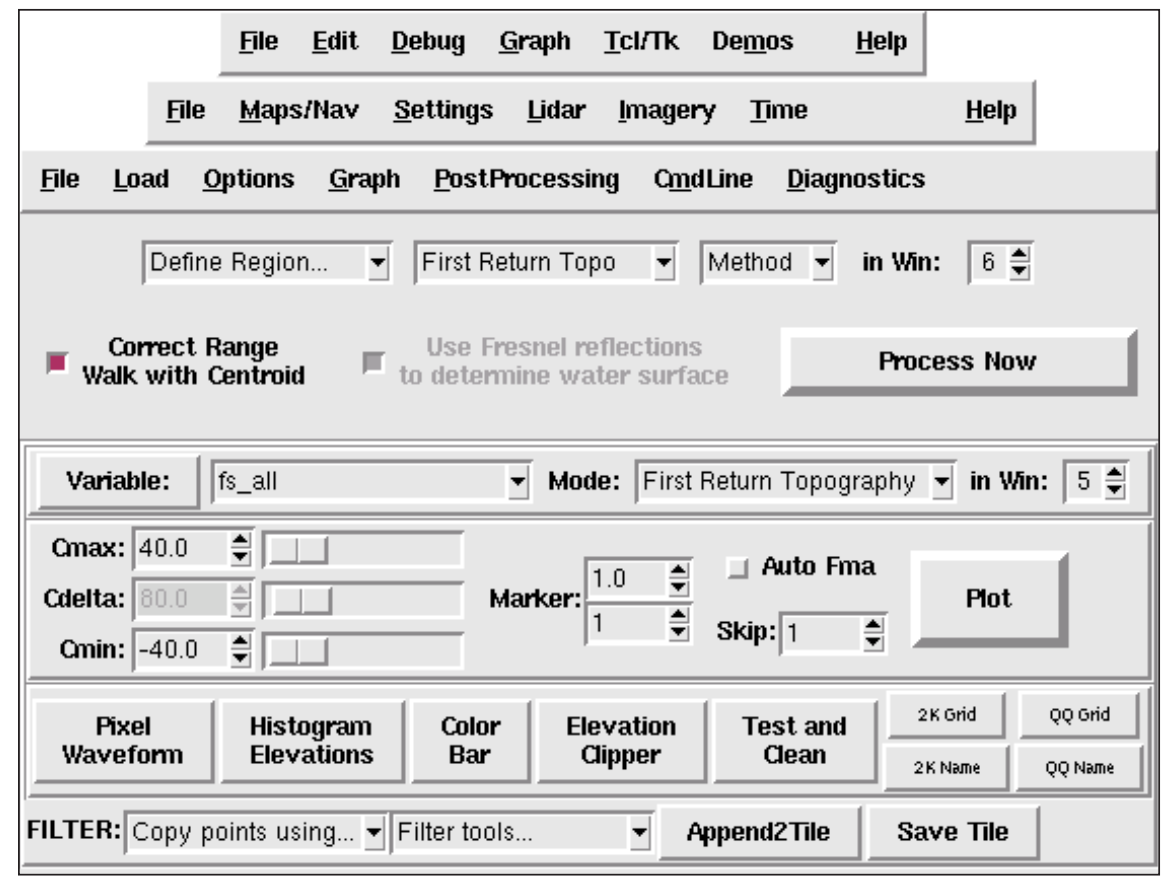

a number of sensor parameters and backscattering properties of targets. Some important sensor parameters include the shape of the laser pulse, the receiver impulse function, and parameters describing pulse spreading (Wagner and others, 2007). Standard pulse-detection methods include threshold (rising edge of signal exceeds given threshold), center of gravity (computed over all points above fixed threshold), maximum zero crossing of second derivative (detection of local maxima), and constant fraction (the zero crossings of the difference between an attenuated and a time-delayed version of the signal) (Wagner and others, 2004).

Problems occur when waveforms have complex forms and backscattered pulses are low when compared to noise levels. Algorithms are adjusted to tasks to account for waveform complexity (Wagner and others, 2004). ALPS uses the following algorithms to differentiate between returns: the zero crossing of the second derivative is used to detect the first return and the trailing-edge algorithm is used to detect the range to the last return; that is, the algorithm searches for the location prior to the last return where the direction changes along the trailing edge (Nayegandhi and others, 2006).

ALPS has the ability to analyze and process the data collected by the EAARL system in an interactive or batch mode. Some interactive modules in ALPS include: pre-survey flight line definition, flight path plotting, topography data generation, lidar raster and waveform examination, and digital camera image playback. ALPS in the batch processing module will automatically generate topography data. This automation eliminates the time-consuming effort of interactively generating topography data.

\section{Interactive Processing Mode}

In order to begin interactive processing, the following steps assume the user is logged into the computer where the raw data are stored. See Appendices A, B, and C for possible steps preceding the initial interactive processing.

\section{Opening ALPS Processing Windows}

Start a terminal session. The user must migrate to the src directory to begin all processing in ALPS.

Type in the command line: cd / opt/eaarl/lidar-processing/src [press enter].

Start yorick session by typing in the command line: ./ytk eaarl.ytk I1pro. ytk [press enter].

Figure 2. ALPS provides an interface for data processing, visualization, and investigation. 
The ytk, eaarl, and Process EAARL Data windows will open. Opening these windows will load a series of files necessary for ALPS functions (fig. 2).

\section{Loading the EAARL Database File}

To load the EAARL Database file (EDB), go to the eaarl window and select file $>>$ Load EAARL Database Index $(E d b)$. The data are stored by mission day within each mission directory. Migrate to the EAARL subdirectory within the selected mission day [for example, /training/raw/HR KATRINA/20050908/eaarl/] and select the idx file [click Open]. After the idx file is loaded the user will see the following: This will set 'gps_time_correction' to - 14.0 (only if the data were collected after 1 January, 2006, otherwise it will be -13.0).

An $e d b$ window will open with information about the dataset. Similar information is listed in the open terminal window (fig. 3). The EAARL window becomes the Current Data window.

A window regarding the digital camera playback will open, Digital Camera Playback Software isn't running. Would

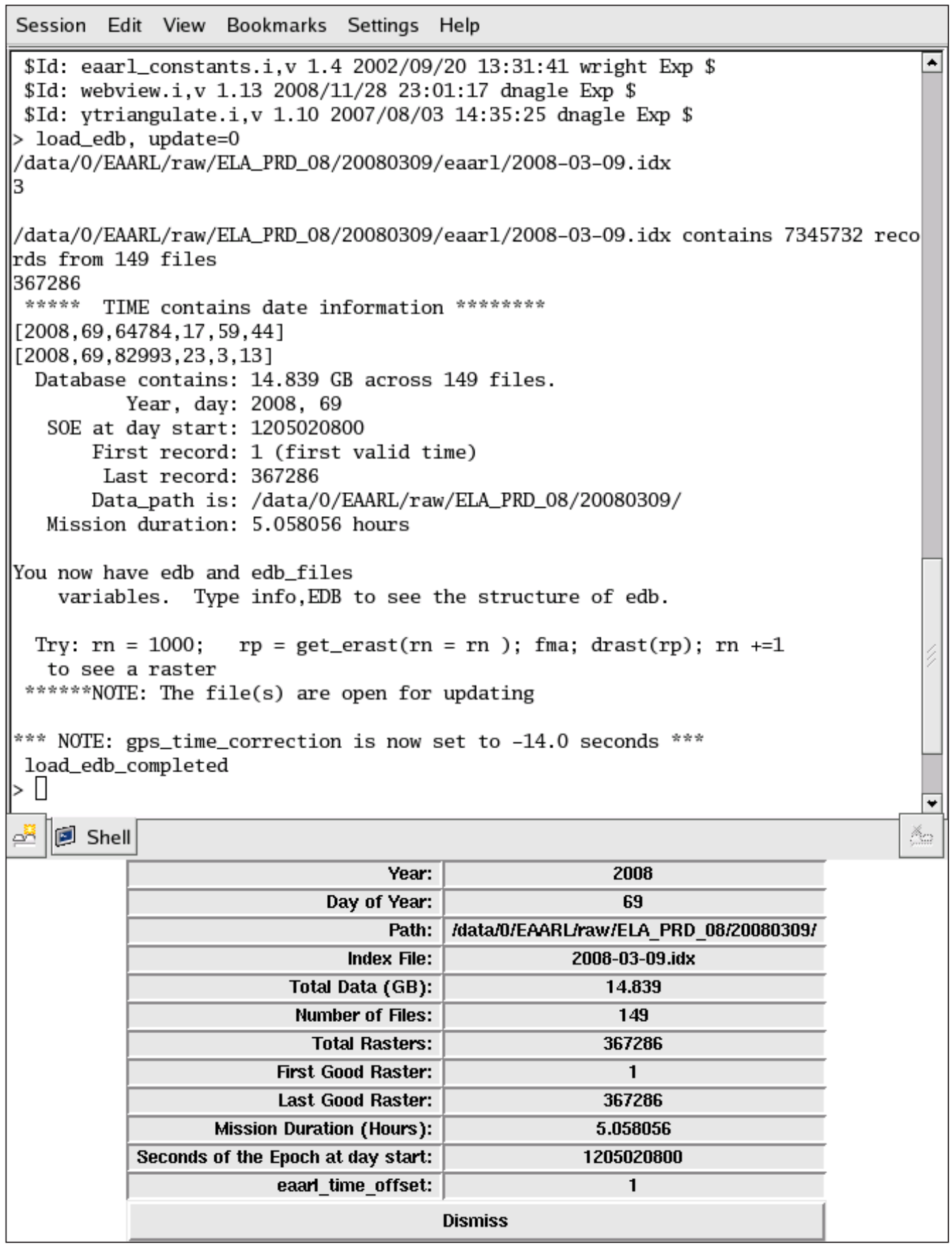

Figure 3. EAARL database index windows. 
you like to run it now? Select Yes. The RGB window appears. To set up the RGB camera to view images acquired during or after the year 2007, go to the $R G B$ window and select File $>>$ Select Path. Select the cam 1 directory [click $0 K$ ]. For any images acquired before 2007, go to the RGB window and select File $>>$ Select File. Select the cam1.tar file within that mission day (/training/raw/HR_KATRINA/20050908/2005-cam1. tar).

A window will open, indicating the camera is mounted. This window will close when all of the tar files are loaded (this may take a minute). This selection will allow the user to pair lidar rasters, waveforms, and images together. Press the forward/backward arrows or drag the crossbar in the RBG window to view the rest of the images collected on that day (fig. 4). The images are collected at an interval of 1 per second(s).

Select Dismiss to close the $e d b$ window. To access the window later, go to the File menu in the Current Data window and select Edb status (fig. 3).

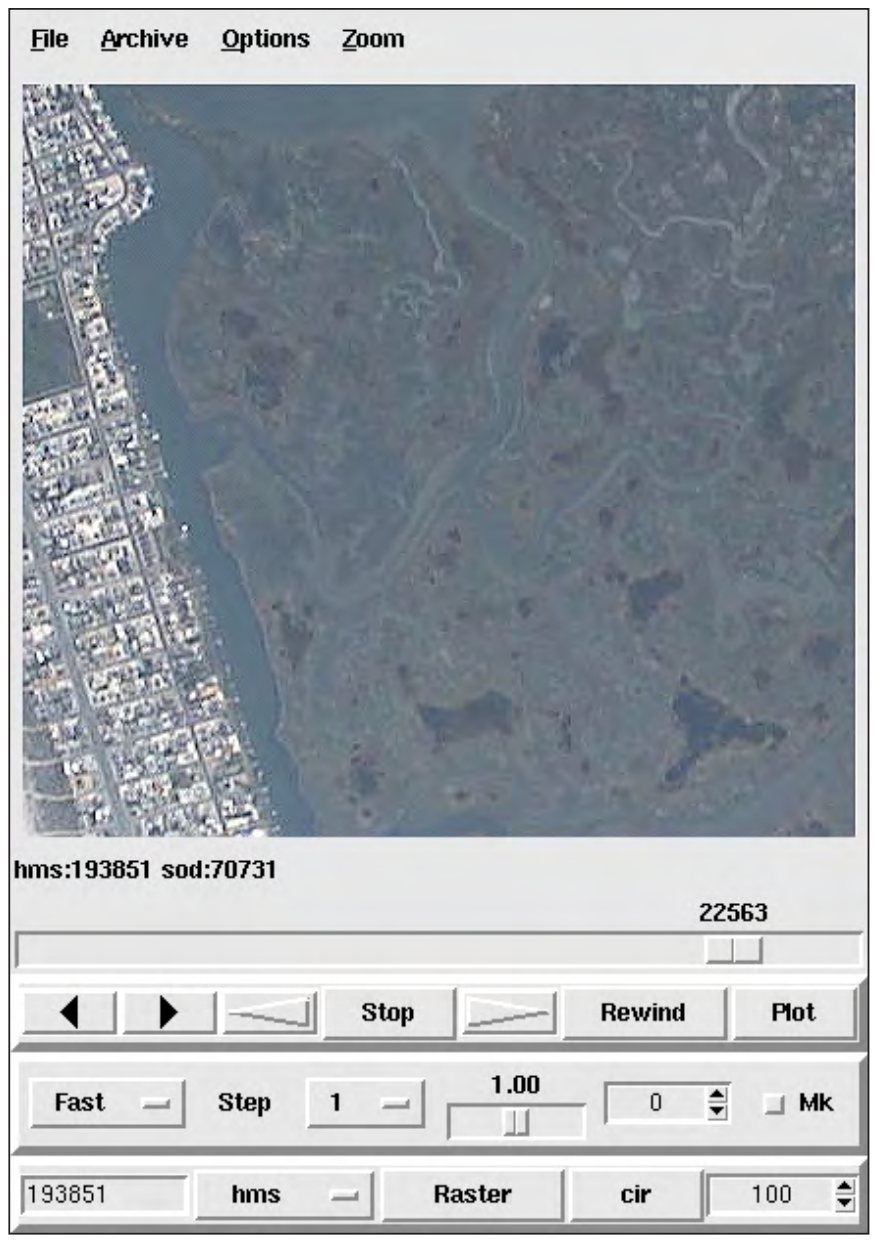

Figure 4. An image from the RGB camera.

\section{Loading GPS Flight Track Information}

To load flight track information, go to the Current Data window and select Maps/Nav >> Plotting Tool. In the window that opens (fig. 5), select the Settings tab and choose a coordinate system by selecting UTM or Lat/Lon in the Coordinates drop-down menu (click to select).

To load the current precision GPS flight track file, go to the Plotting Tool window (fig. 5) and select the PNAV tab $>>$ Load Track. The flight day directory window will open. Select the trajectories directory. This trajectories directory window can also be opened by going to the File menu of the Current Data window and selecting Load GPS PNAV data. Select the most recent $c m b$-pnav directory (with $p$ in title) [click Open].

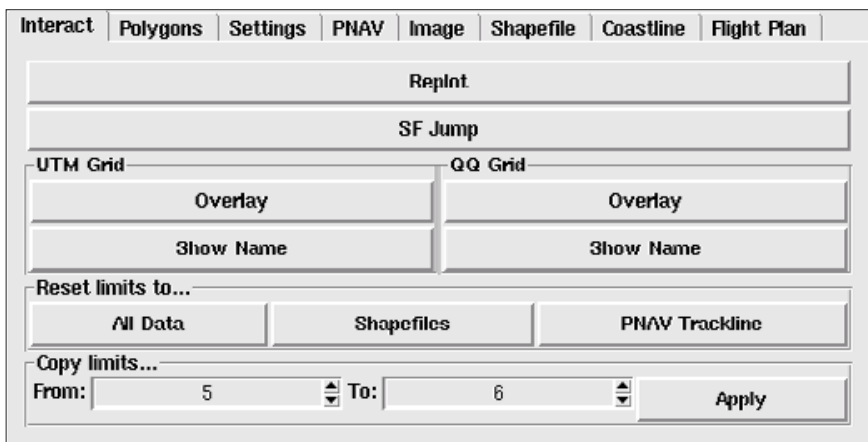

Figure 5. The Plotting Tool window manages flight lines, shapefiles, coastal base maps, and flight plans.

The naming scheme of the selected GPS file should be similar to the following example: YYYY-MM-DD-p-YYYY-MMDD-zny1-wgs84-cmb-pnav (table 1). When the pnav directory window opens, select the .ybin file [click Open]. If a precision pnav is not selected, then a warning window will appear stating: The pnav file you have selected does not appear to be a precision trajectory. It should not be used in the production of final data products or to assess accuracy of the system.i. The precision pnav file accounts for all of the errors in the GPS system. See www.ngs.noaa.gov/orbits or http://igscb.jpl.nasa. gov/igscb/center/analysis/noaa.acn for further details on GPS processing.

Table 1. GPS satellite ephemeride file indicators.

\begin{tabular}{|l|l|l|}
\hline GPS Solution & $\begin{array}{l}\text { Availability From Last } \\
\text { Observation }\end{array}$ & Accuracy \\
\hline U - Ultra & 6 hours & $\sim 20-40 \mathrm{~cm}$ \\
\hline R - Rapid & 17 hours & $\sim 4-7 \mathrm{~cm}$ \\
\hline B - Broadcast & 24 hours & $\sim 1.6 \mathrm{~m}$ \\
\hline P - Precision & $4-10$ days & $\sim 2-5 \mathrm{~mm}$ \\
\hline
\end{tabular}




\section{Loading Digital Miniature Attitude Reference System (DMARS) Information}

Next, the Digital Miniature Attitude Reference System (DMARS) must be loaded. This Inertial Measurement Unit (IMU) is used for precise-attitude determination. Load the information from the Current Data window by selecting File $>>$ Load DMARS Attitude data or $>>$ Maps/Nav $>>$ Load DMARS data. The trajectories window will appear again. Select the most recent -ins directory [click Open]. When the ins directory window opens, select the ins.pbd file [click Open].

The command window should reflect all data that is loaded (fig. 6). The applied GPS correction time, minimum and maximum values for the Seconds of the Week (SOW), Position Dilution of Precision (PDOP), Root Mean Square Error (RMSE), latitude, longitude, and altitude are shown. All loaded files are also listed.

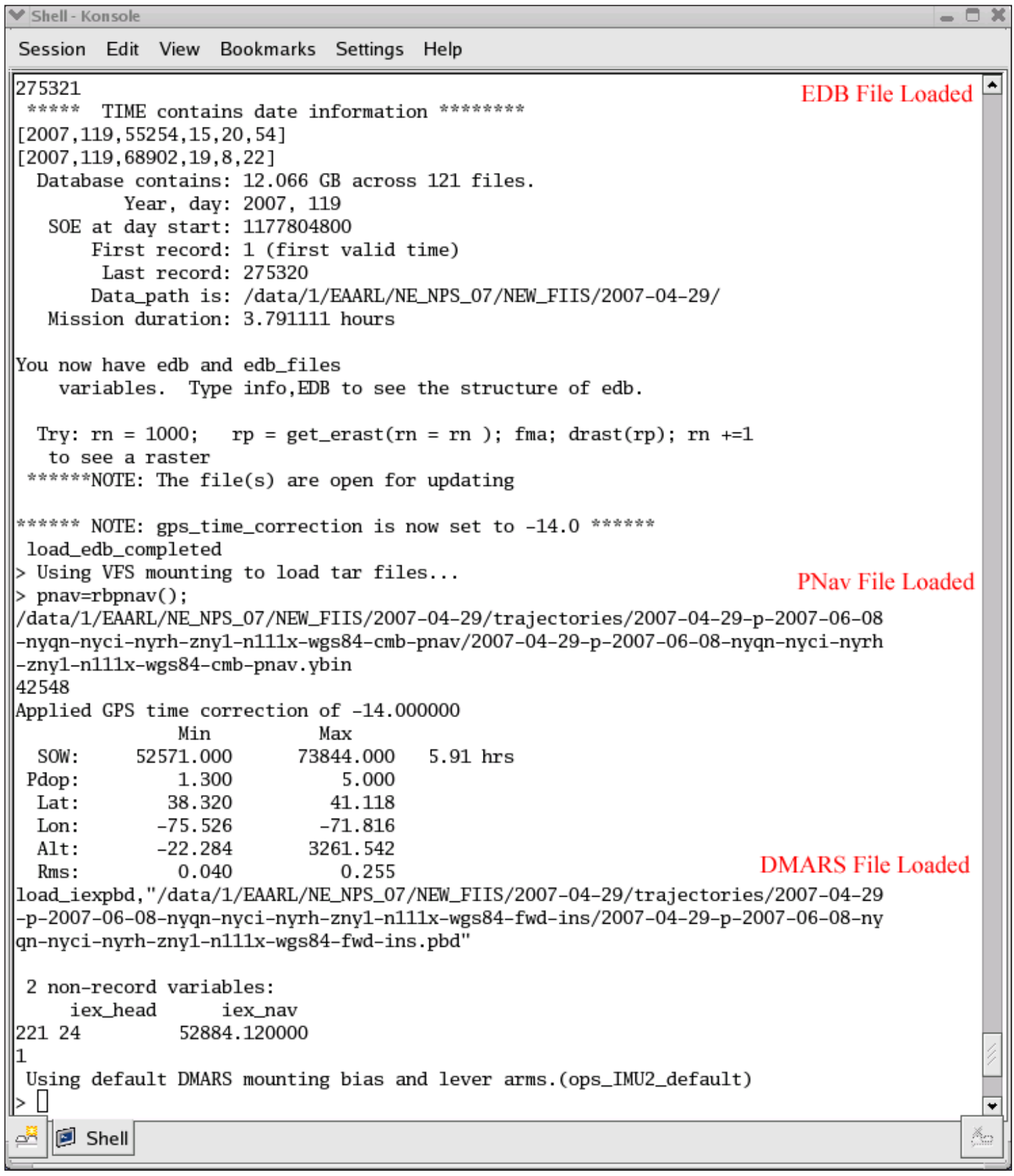

Figure 6. Yorick shell after the EDB, PNav, and DMARS files have been loaded. 
Position Dilution of Precision is the measure of the geometrical strength of the GPS satellite configuration. This is the amount of error in the GPS position. A PDOP less than 4 results in the best accuracy (under 1 meter). A PDOP higher than 4 may be displayed, but it is likely the EAARL system was not collecting data at that time.

\section{Loading Mission Constants}

To load mission constants, go to the Current Data window and select Settings $>>$ Load Ops_conf Settings. A window will open showing the current directory. Select the ops_conf-YYYYMMDD.i file [click Open]. If an ops_conf file does not exist for each day of flight, navigate to a different directory to select the file (usually up one directory, to the mission directory). This indicates only one ops_conf file was created for the entire mission. To determine the loaded mission constants, type ops_conf in the command line or in the Current Data window, select Settings $>>$ Current Ops_conf Settings.

\section{Viewing Flight Lines}

To view flight lines, click Plot Track on the PNAV tab on the Plotting Tool window (fig. 7). On the Yorick 6 window, left

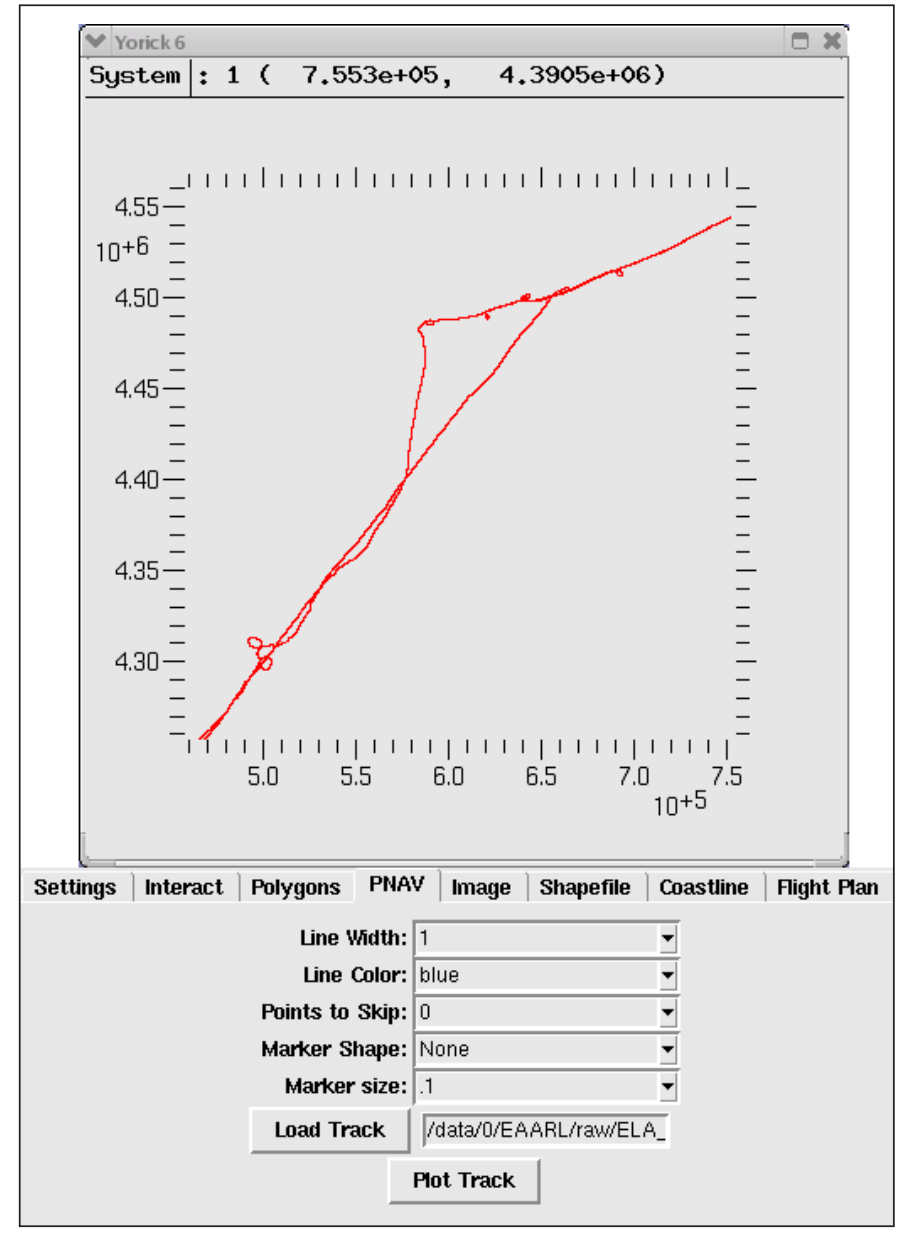

Figure 7. Flight lines window. click to zoom in and right click to zoom out. Middle click (or control left click) and hold to pan. Customize the flight line view by using the Plotting Tool window (fig. 5) to adjust the line width, line color, points to skip, marker shape, and marker size (table 2). Some collection days will cross Universal Transverse Mercator (UTM) zones, and that flight line data will not plot in the window. In this case, the shell will inform the user that Selected flight line crosses UTM Zones. To view flight lines from a specific UTM zone, type curzone=\# [for example, curzone=16] in the command line. Only that portion of the flight lines collected in that zone will plot in the Yorick 6 window.

\section{Loading Coastal Base Maps}

To load coastal base maps, click Add coastline map under the Coastline tab of the Plotting Tool window. This will

Table 2. Interact tab for the Plotting Track window functions.

\begin{tabular}{|c|c|c|}
\hline Feature & Functionality & $\begin{array}{l}\text { Command Line } \\
\text { Functions }\end{array}$ \\
\hline Reset Limits to... & $\begin{array}{l}\text { Resets the viewing } \\
\text { window to all data, } \\
\text { shapefiles, and } \\
\text { PNAV tracklines. }\end{array}$ & $\begin{array}{l}\text { Another way to } \\
\text { restore all of the } \\
\text { loaded information } \\
\text { on the active } \\
\text { window is to type } \\
\text { limits or window, } \\
\text { \#; limits [window, } \\
\mathbf{1 ;} \text { limits]. }\end{array}$ \\
\hline Clear and Plot & $\begin{array}{l}\text { Refreshes the } \\
\text { selected window. }\end{array}$ & \\
\hline SF Jump & $\begin{array}{l}\text { Allows the user to } \\
\text { select to point on } \\
\text { the displayed flight } \\
\text { line. The command } \\
\text { terminal will list } \\
\text { information about } \\
\text { the selected area. } \\
\text { The Red-Green- } \\
\text { Blue (RGB) camera } \\
\text { image will jump to } \\
\text { this selected area. }\end{array}$ & $\begin{array}{l}\text { Set the Universal } \\
\text { Transverse } \\
\text { Mercator (UTM) } \\
\text { zone by typing } \\
\text { curzone=\# in the } \\
\text { command terminal } \\
\text { [for example, } \\
\text { curzone=16]. }\end{array}$ \\
\hline Overlay & $\begin{array}{l}\text { Displays a UTM or } \\
\text { quarter quadrangle } \\
\text { grid over the data. }\end{array}$ & \\
\hline Show Grid Name & $\begin{array}{l}\text { Lists given UTM or } \\
\text { quarter quadrangle } \\
\text { grid name. After } \\
\text { the grid has been } \\
\text { overlaid onto data, } \\
\text { click on the active } \\
\text { data window. }\end{array}$ & \\
\hline Copy limits... & $\begin{array}{l}\text { Apply the limits from } \\
\text { one window to } \\
\text { another. }\end{array}$ & \\
\hline
\end{tabular}


provide a visual map of the flight line locations. Select the $p b d$ directory (/opt/eaarl/lidar-processing/maps/pbd/) to see a list of .pbd files from around the world. Pick the appropriate file depending on the region from which the data were collected. For example, choose med-res-eusa.pbd for the Long Island area. Select fla.pbd for the State of Florida coastline map. Once the coastline is chosen, click Plot coastline maps. The following information may appear on the command window: Selected Base Map Crosses \# UTM Zones. Select Zone Number from \# to \#: Enter Zone Number. Input a zone number here [for example, 16] and press enter. The Yorick 6 window will load the base map. To change zones, go to the Interact tab and click Clear and Plot.

\section{Viewing Raster Images and Waveforms}

To load the raster images, go to the Lidar menu on the Current Data window and select Examine Lidar Rasters. The drast window will appear. Click Raster on the $R G B$ camera window. This will display an unreferenced raster image for that area (fig. 8). The field of view of the RGB camera slightly exceeds the length of the raster scan. A raster represents one side of the oscillating scan (one zig or one zag) (fig. 9). Using the drast window, the user can press play or drag the crossbar to view the rest of the raster images collected. Click the Animate GeoRefbutton to obtain the georeferenced raster image for that particular RGB image displayed (fig. 8).

To analyze waveforms for the unreferenced raster image, click Examine Waveforms in the drast window. The command window will state Window: 1. Left Click: Examine Waveform. Middle click: Exit. By left clicking on the raster image in several locations, the user will see that waveforms change.

\section{Loading Other Images}

Rather than overlaying the flight lines onto a base map, the user may want to overlay the flight lines onto an image. To load other images, go to the Image tab in the Plotting

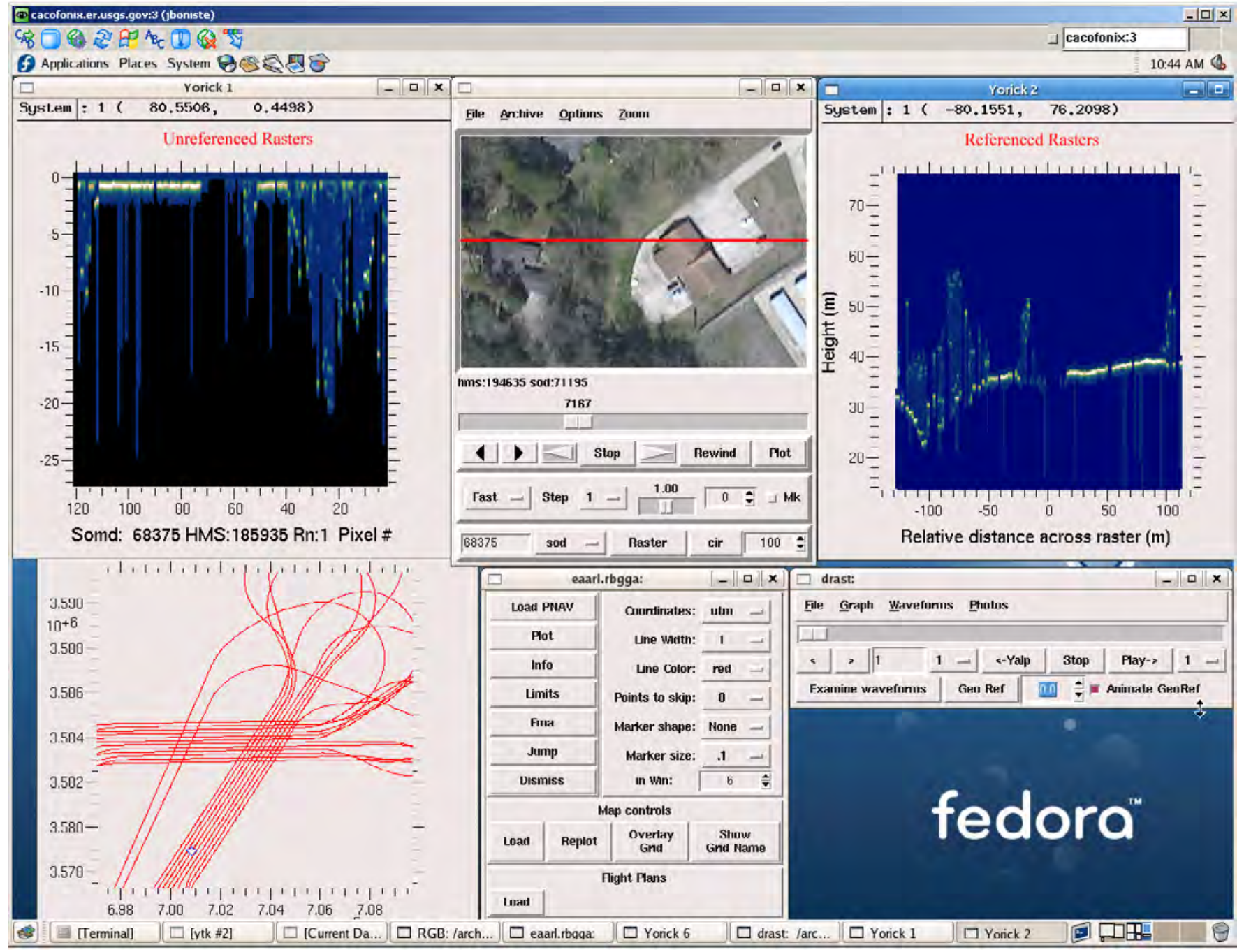

Figure 8. Screenshot from an interactive session in ALPS showing EAARL rasters (top right and left), RGB (top center) images, and flight line map (bottom left). 


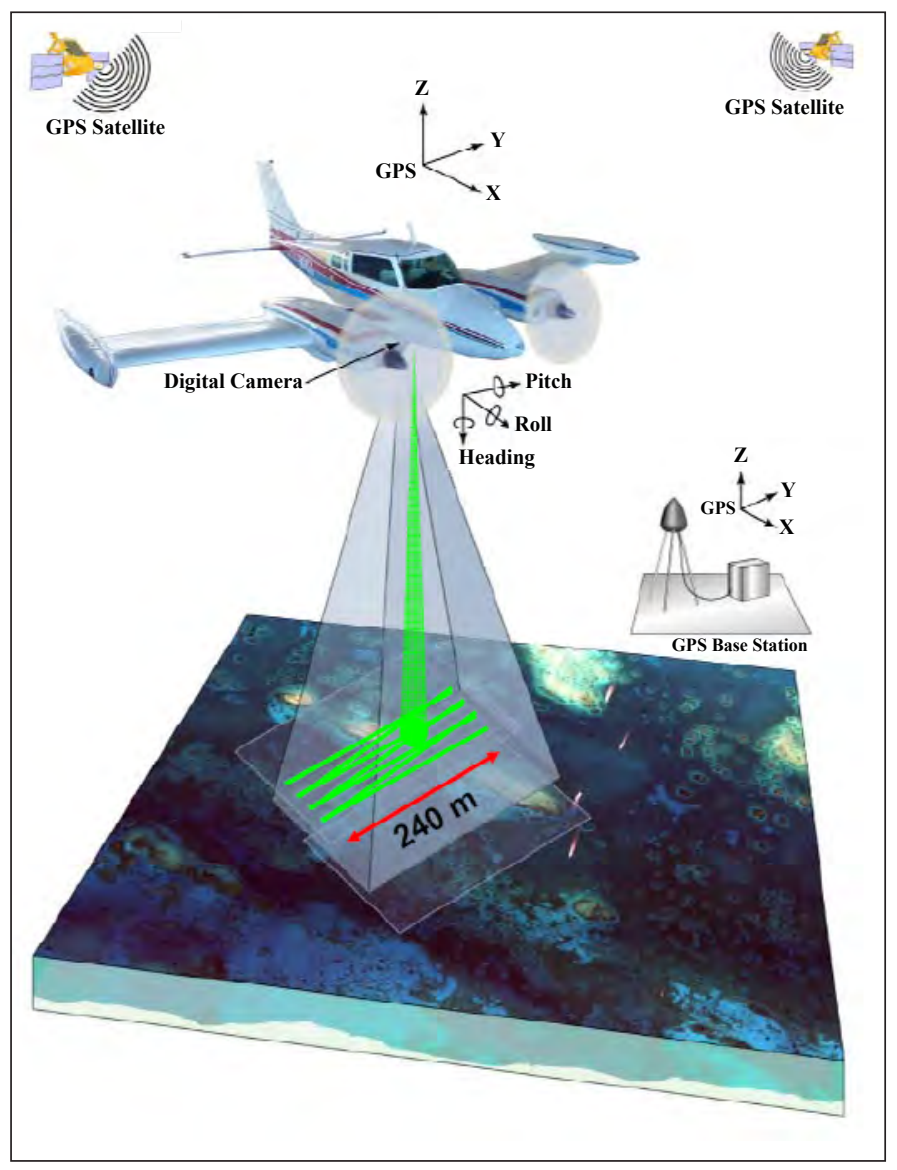

Figure 9. An illustration of the EAARL oscillating scan pattern and aircraft positioning (Brock and others, 2004).

Tool window. Select Add image with world file, Add image, specifying location, or Add lidar image. In the new window that opens, navigate to the directory that contains the image. It will automatically search for .jpg, but .png, .gif, and .tif files can also be viewed by changing the file type at the bottom of the window.

Click Plot Images in order to view the image in the defined Yorick 6 window location.

\section{RGB Camera Functions}

To view and manipulate the RGB images, follow the instructions in table 3.

\section{CIR Camera Functions}

To load and view CIR images, go to the Current Data window and select 1-Hz CIR High-Res Images from the Imagery menu. A window will open that allows the user to navigate to the directory where the CIR images are located. Select the cir directory within the mission day directory [click OK]. A blank window will appear. To view the CIR images, move the slider bar to a point in the flight on the RGB window. Then click on the cir button in the RGB window.
Table 3. Red-Green-Blue (RGB) camera functions.

\begin{tabular}{|c|c|}
\hline Feature & Functionality \\
\hline Arrow keys or slider bar & $\begin{array}{l}\text { Moves through the flight line } \\
\text { images (fig. 4). }\end{array}$ \\
\hline 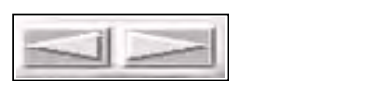 & $\begin{array}{l}\text { Plays continuously through the } \\
\text { RGB images. }\end{array}$ \\
\hline Stop & Ends continuous play. \\
\hline Rewind & $\begin{array}{l}\text { Returns the viewer to the first } \\
\text { image of data collection. }\end{array}$ \\
\hline Plot & $\begin{array}{l}\text { Shows the user where the image } \\
\text { is located in the flight line data. } \\
\text { In the window where the flight } \\
\text { lines are displayed, a blue } \\
\text { triangle will appear (fig. 7). }\end{array}$ \\
\hline Fast & $\begin{array}{l}\text { Changes speed at which the } \\
\text { images are continuously } \\
\text { viewed. }\end{array}$ \\
\hline Step & $\begin{array}{l}\text { Determines how many images are } \\
\text { skipped at once. For example, } \\
\text { if ' } 2 \text { ' is selected, then the user } \\
\text { will skip ahead or back two } \\
\text { images at a time. }\end{array}$ \\
\hline 1.00 & $\begin{array}{l}\text { Moving the slider bar will alter } \\
\text { the brightness of the image. }\end{array}$ \\
\hline 】 & $\begin{array}{l}\text { This function is located next to } \\
\text { the Step function. }\end{array}$ \\
\hline $0 \quad \boldsymbol{\nabla}$ & $\begin{array}{l}\text { Syncs RGB and Color-Infrared (CIR) } \\
\text { images if they are displayed } \\
\text { separately from one another. }\end{array}$ \\
\hline Mk & $\begin{array}{l}\text { Marks a particular image. Can } \\
\text { move through only the marked } \\
\text { images. }\end{array}$ \\
\hline hms - & \multirow{2}{*}{$\begin{array}{l}\text { Can select specific RGB images } \\
\text { by entering in a value in this } \\
\text { space. Depress the 'hms' button } \\
\text { to display the value in hours, } \\
\text { minutes, and seconds (hms), } \\
\text { seconds of the day (sod), or } \\
\text { image index (cin). }\end{array}$} \\
\hline & \\
\hline Raster & $\begin{array}{l}\text { Pairs an RGB image with a raster } \\
\text { scan. }\end{array}$ \\
\hline CIR & $\begin{array}{l}\text { Pairs CIR images with RGB } \\
\text { images. }\end{array}$ \\
\hline $100 \stackrel{-}{*}$ & $\begin{array}{l}\text { Changes the zoom factor of } \\
\text { the RGB image. The lowest } \\
\text { possible value is ' } 1 \text { ' and the } \\
\text { highest possible value is ' } 200 \text { '. }\end{array}$ \\
\hline
\end{tabular}

The image in the RGB window will then appear in the blank window (fig. 10). The CIR images can be viewed and manipulated as follows (table 4). 


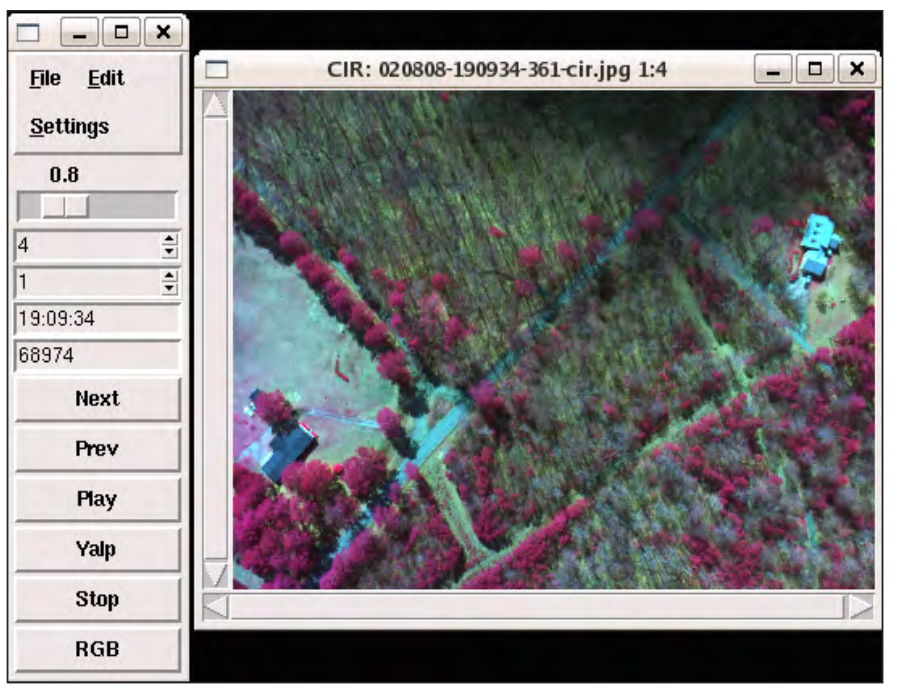

Figure 10. An image from the CIR camera.

\section{Topographic Lidar Data Processing}

To begin the processing of EAARL lidar data, the user must zoom in on the flight lines shown in the Yorick 6 window. Select a small area with at least two flight lines. Select flight lines for processing by going to the Define Region drop-down menu in the top panel of the Process EAARL Data window. Select one of the following choices:

Points in Polygon - This function is used to select any kind of polygonal shape. This method is the easiest way to select as little or as much of a desired area as is needed.
Rubberband Box - This function will automatically create a rectangular-shape box from the northwest corner when dragged across the screen.

Rectangular Coords - This function will process a specific area with known latitude/longitude or UTM coordinates (fig. 11).

If the Points in Polygon method is selected, a message appears as follows, Draw a Polygon in Window 6 to define a region using a series of left mouse clicks. To complete the polygon, middle mouse click or <Ctrl> and left mouse click. [click $O K$ ]. To select an area, complete a series of left clicks that creates a polygon and center click to finish the polygon.

If the Rubberband Box method is selected, a message appears as follows, Drag a Rectangular Box in Window 6 to define region. [click $O K$ ]. To select an area, left click, drag across the flight lines, and then release to complete the box.

If the Rectangular Coords method is selected, the /1widc window will open. Select either LatLon or UTM from the Coordinate System drop-down menu. Type in the UTM Zone,

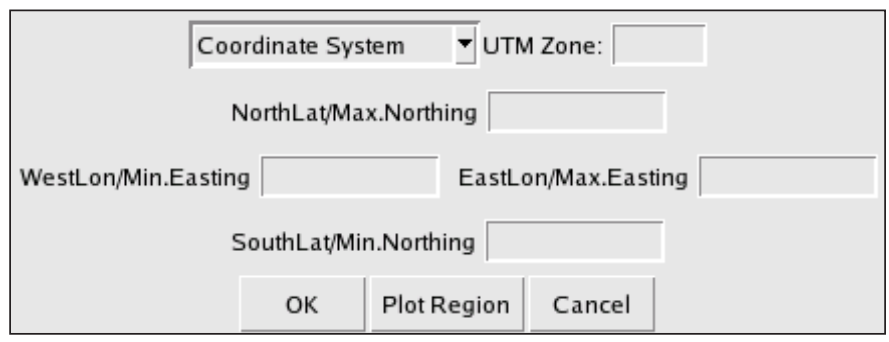

Figure 11. Rectangular coordinate (I1widc window) method.

Table 4. Color-Infared (CIR) camera functions.

\begin{tabular}{|c|c|}
\hline Feature & Functionality \\
\hline Center or right click on the CIR image. & Opens CIR menu. \\
\hline Left click on CIR image. & Moves onto the next collected image (fig. 10). \\
\hline \begin{tabular}{|c|}
$\mathbf{1 . 0 0}$ \\
$\perp$ \\
\end{tabular} & Alters the brightness of the image. \\
\hline 4 & $\begin{array}{l}\text { Controls the size of the image. A value of ' } 15 \text { ' yields the smallest image while a value of } \\
\text { ' } 1 \text { ' yield the largest image. Located beneath the slider bar. }\end{array}$ \\
\hline 1 & Changes the number of images to skip. \\
\hline 19:09:34 & Shows when the image was collected in flight in hms as well as sod. \\
\hline Next & $\begin{array}{l}\text { Moves forward through the images. It will move through the number of selected skipped } \\
\text { images. }\end{array}$ \\
\hline Prev & $\begin{array}{l}\text { Moves backward through the images. It will move through the number of selected } \\
\text { skipped images. }\end{array}$ \\
\hline Play & $\begin{array}{l}\text { Moves forward continuously through the images. It will move through the number of } \\
\text { skipped images. }\end{array}$ \\
\hline Yalp & $\begin{array}{l}\text { Moves backward continuously through the images. It will move through the number of } \\
\text { skipped images. }\end{array}$ \\
\hline RGB & Aligns the RGB image with the selected CIR image. \\
\hline
\end{tabular}


North Lat/Max.Northing, South Lat/Min.Northing, West Lon/ Min.Easting, and East Lon/Max.Easting. Click Plot Region (Click OK).

If the Points in Polygon or Rubberband Box method is selected, draw a polygon or a box over the flight lines. Once drawn, the command window will list how many records and seconds of data are included. Processing time increases substantially if the selected area is too large. An area that includes less than $300 \mathrm{~s}$ of data is most efficient.

Next, select Topo Under Veg in the data type dropdown menu, located next to the Define Region drop-down menu. This mode is chosen unless processing for submerged topography. (See the Submerged Topography (Bathymetry) Lidar Data Processing with Waveform Investigation section for processing details.) Topo Under Veg processes both first and last returns. Other choices for processing the data include Multi Peak Veg and Direct. Wave Spectra. (See appendix E for discussion on these processing choices.)

It is not necessary to use the Method drop-down menu or the in Win: menu in the top panel of the Process EAARL Data window. Keep the Correct Range Walk with Centroid selected. Click the Process Now button. The user will observe a Flight Segment Progress Bar while the command window lists the details of the topographic data processing. A return $[>]$ will appear when the topographic data processing has finished.

\section{Viewing Processed Topography Data}

To view processed topography data, go to the Process EAARL Data window to choose one of the variables from the Variable drop-down menu in the middle panel. Select veg_all for viewing first or last returns. Choose First Return Topography or Bare Earth Topography, respectively, in the Mode drop-down menu. Other Mode options include:

- Surface Amplitude - This mode shows the backscatter from the first waveform channel.

- Bottom Amplitude - This mode shows the backscatter from the last waveform channel.

- Canopy Height - This mode represents the distance from the first return to the bare ground.

Do not adjust the in Win: drop-down menu in the middle panel of the Process EAARL Data window. Click the Auto Fma button to plot with a fresh screen each time the Plot button is pressed. Click the Histogram Elevations button. The Yorick 7 window will open with a histogram displaying the elevation range of data in WGS84 (G1150) elevations (fig. 12). The Color Bar Tool window will also open. On the Color Bar Tool, select the Both button. Click the location on the histogram that corresponds to the minimum elevation, $\mathrm{Cmin}$, and then choose the maximum elevation, Cmax. The minimum elevation can be selected separately by clicking $\mathrm{Cmin}$ and selecting the location on the histogram. The same can be done to select a location for maximum elevation, Cmax. Click Plot and a window will open with a view of processed data. These data are unfiltered, so noise may be visible. Elevations higher than Cmax will appear white, while elevations lower than Cmin will not appear.

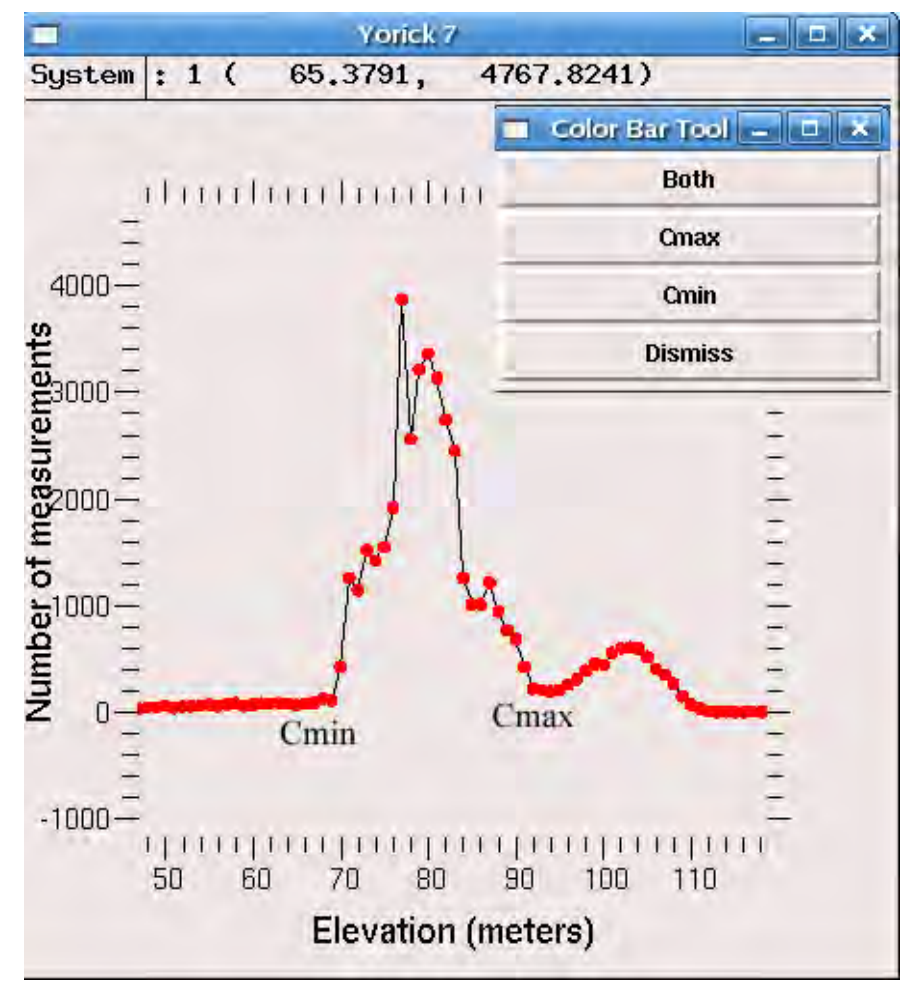

Figure 12. A histogram display and color bar tool window.

To link the processed topography data with the waveforms, raster images, and RGB images, press the Pixel Waveform button in the middle panel of the Process EAARL Data window. Left click on the window with the processed topography data to examine a point, center click to set as a reference point, and right click to quit. When left clicking over the topography data points, two windows will open. One will display the waveform for that data point and another will display the raster image (fig. 13). The waveform, raster, and the RGB camera windows are all georeferenced together. Right click on the processed topography data to quit the pixel waveform module.

The sample waveform window in figure 13 represents a sandy region with coastal vegetation. The display shows all three channels: black (90\%), red (9\%), and blue $(0.9 \%)$. The $\mathrm{x}$-axis displays digital counts, which represent the amplitude or relative backscatter. The digital count values are inverted in the EAARL receiver hardware. The values decrease with an increase in backscatter return signal strength (Nayegandhi and others, 2006).

The raster scan consists of 120 laser pulses along a zig or zag approximately normal to the flight path. The raster is a false-color image of the backscatter return signal for the most sensitive channel. Bright colors indicate high signal strength. The vertical stripes in the scan indicate that the laser pulse does not have recorded data (Nayegandhi and others, 2006). 


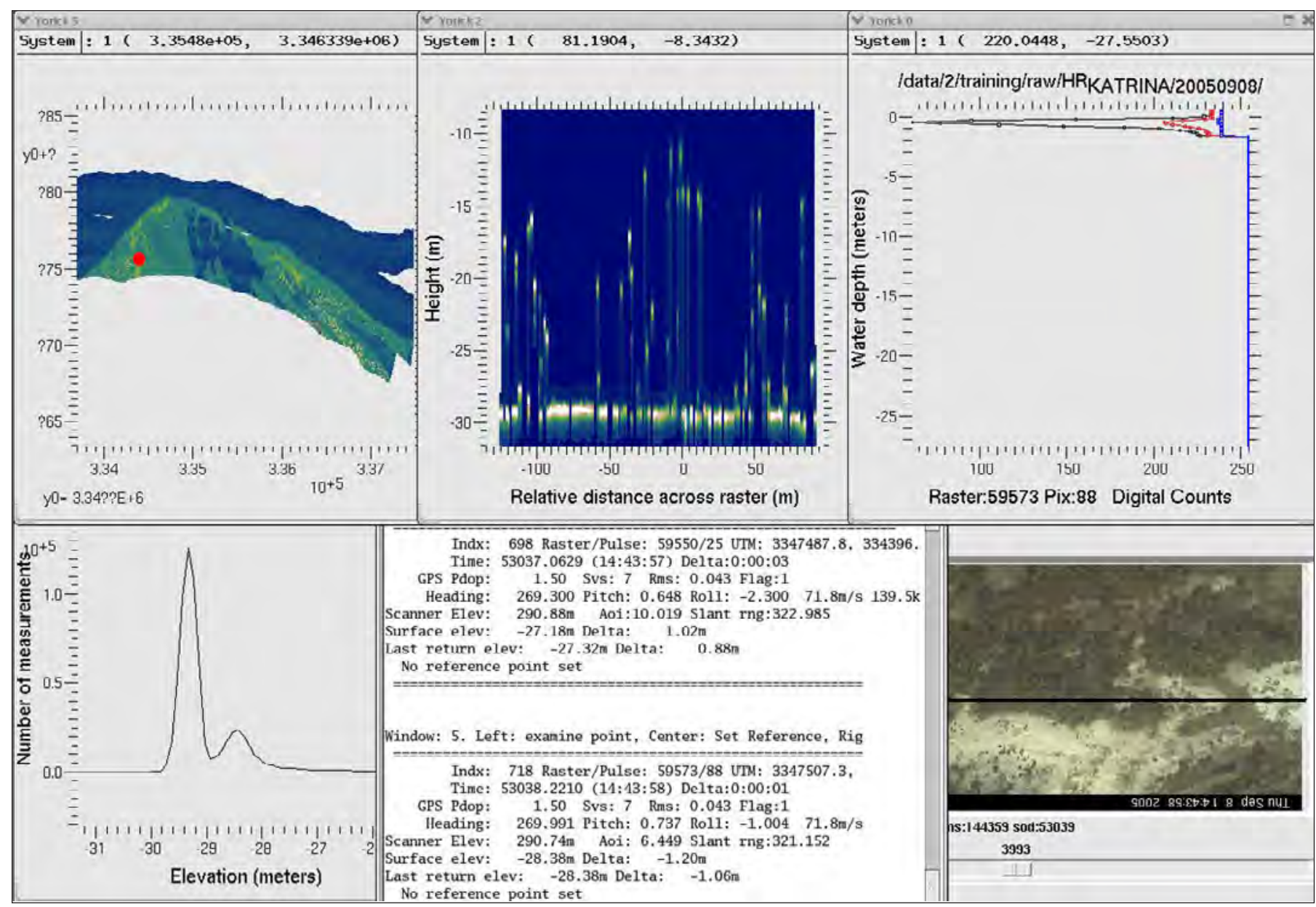

Figure 13. A screenshot showing processed data (top left), a georeferenced raster image (top center), a sample waveform (top right), a histogram (bottom left), the ALPS command window (bottom center), and an RGB image (bottom right).

\section{Submerged Topography (Bathymetry) Lidar Data Processing with Waveform Investigation}

Water surface specular reflection (Fresnel reflection) usually occurs near nadir, along the scan pattern. Fresnel's equations describe the behavior of light when it moves between media of differing refractive indices. These reflections are represented by a strong-amplitude backscatter signal, usually saturated in the most sensitive channel, while the range is then resolved in the least sensitive channel. The water surface elevation is estimated by determining the range of all Fresnel reflections within a scan and extrapolating these values for the entire length of scan. The sea bottom is determined by correcting for the following: refraction of the incident laser pulse at the air-water interface, change in the speed of light as it enters the water column, the attenuation of the laser signal in water, and the effect of water column turbidity on the laser backscatter. To determine the bottom, corrections are performed by examining the sample waveforms from different locations in the survey area to define constants for exponential decay. The data are processed in reference to the WGS84 (G1150) ellipsoid datum, which is independent of the elevation of the water surface (Nayegandhi and others, 2006).

Interactive processing for submerged topography is similar to topographic processing with a few adjustments and additions. Complete the previous steps in the Interactive Processing Mode sections. Also review the Topographic Lidar Data Processing and the Viewing Processed Topography Data sections of this manual for the following repeated steps.

Zoom in to a small area of the loaded flight lines in the Yorick 6 window. Select the flight lines by going in the Define Region drop-down menu on the Process EAARL Data window. Select one of the following tools:

- Points in Polygon

- Rubberband Box

- Rectangular Coords

Once a tool is chosen, a window will open discussing the selection. If the Points in Polygon tool was selected, draw a polygon over the flight lines. Select Submerged Topo in the drop-down menu located next to the Define Region dropdown menu. An alternate method for viewing the submerged 
topography data is also available. See appendix E for details on the Direct. Wave Spectra mode.

It is not necessary to use the Method drop-down menu or the in Win: menu. Keep Correct Range Walk with Centroid and Use Fresnel reflections to determine water surface buttons selected. Use Fresnel reflections to determine water surface should not be selected in riverine or coastal environments; when the lidar scan regularly crosses the land-water interface.

Load the bathymetry settings by selecting Load Bathymetry Settings from the Load menu in the Process EAARL Data window. The bathct/ window will open (fig. 14) with a list of variables (table 5).

Table 5. Submerged topography settings.

\begin{tabular}{|l|l|}
\hline \multicolumn{1}{|c|}{ Feature } & \multicolumn{1}{c|}{ Functionality } \\
\hline Laser & $\begin{array}{l}\text { Estimates exponential decay. The value is } \\
\text { represented by the magenta line. }\end{array}$ \\
\hline Water & $\begin{array}{l}\text { Represents the exponential decay over the water } \\
\text { column. The value is represented by the gray } \\
\text { exponential curve. }\end{array}$ \\
\hline AGC & $\begin{array}{l}\text { Is the exponential equalizer. The value is } \\
\text { represented by the magenta line. }\end{array}$ \\
\hline Thresh & $\begin{array}{l}\text { Represents a threshold number. The value above } \\
\text { this number will be a "good" return and below } \\
\text { this value will be considered noise. This value is } \\
\text { represented by a red straight line that connects } \\
\text { first and last lines. }\end{array}$ \\
\hline First & $\begin{array}{l}\text { The algorithm begins looking for a first surface } \\
\text { return at this value. The value is represented by } \\
\text { the left-hand green line. }\end{array}$ \\
\hline Max. Sat & $\begin{array}{l}\text { The algorithm stops looking for a bottom return at } \\
\text { this value. The value is represented by the right- } \\
\text { hand red line. }\end{array}$ \\
\hline return that is acceptable.
\end{tabular}

Click the Process Now button. The user will observe the Flight Segment Progress Bar while the command window will list the details of the submerged topography data processing. A return [ $>$ ] will appear when the submerged topography data processing has finished. Go to the Process EAARL Data window to view the processed data. Choose depth_all from the Variable drop-down menu. Choose Submerged Topography from the Mode drop-down menu. Do not change the in Win menu. Click the Auto Fma button to refresh the plot window each time a plot is complete. Click the Histogram Elevations button. Select the Both button on the Color Bar Tool. Click the locations on the histogram that correspond to the minimum [Cmin] and maximum [Cmax] elevations. Click Plot and a window will open with a view of the processed data. These data are unfiltered, so noise may be visible. Elevations higher

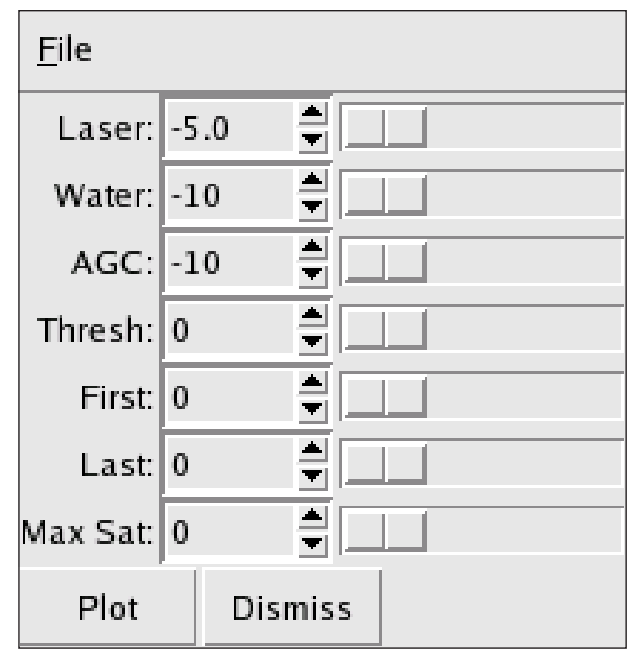

Figure 14. Submerged topography settings window.

than Cmax will appear white, while elevations lower than Cmin will not appear.

To link the processed submerged topography data with the waveforms, raster images, and RGB images, go to the Options menu on the Process EAARL Data window. Select the Show raw and processed waveforms when using Pixel Waveform. Press the Pixel Waveform button. Left click on the data window to examine a point, center click to set as a reference point, and right click to quit the pixel waveform module. When left clicking over the data points, three windows will open. One will display the raw submerged topography waveform for that data point (fig. 15), one will show the processed waveform, and a third window will display the raster image.

The sample raw waveform window in figure 15 represents a shallow, submerged topography region. The waveform window in figure 15 is showing the selection of the bottom return.

Each of the controlling variables is listed in figure 14. If the variables are changed to such a degree that a bottom return is not selected, the terminal window and the raw waveform window will state the reason the bottom was not selected (Below threshold). Every time a variable is altered, the alteration is saved by ALPS, and final settings will be applied to all of the submerged topography processing.

\section{Determining the Roll Bias}

System installation angles, aircraft orientation angles, and the resulting beam angle are examples of calibration parameters set up for each lidar system (fig. 16). These dimensions usually remain stable; however, over time or whenever the system is removed from the surveying platform, the biases can change significantly. Knowing these parameters on a flight-by-flight basis is important because erroneous values can result in users misinterpreting data as a "signal" instead of noise (Shrestha and others, 2007). 


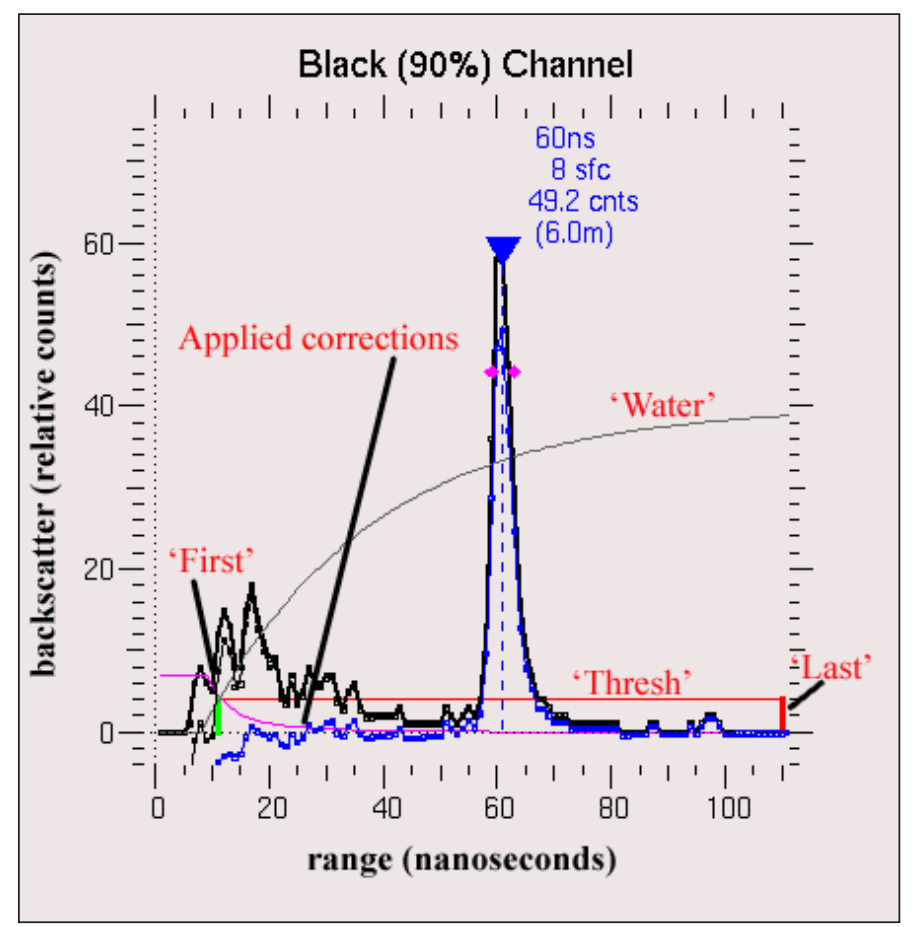

Figure 15. Submerged topography waveform display with the raw waveform (black) and the processed waveform (blue) after the submerged topography settings are applied.

All of the mission constants are set up in the ops_conf.i file. The only constant that is usually adjusted is the roll bias. To determine the constant values, either type in the command line ops_conf or go to the Current Ops_conf Settings submenu within the Settings menu in the Current Data window (fig. 16).

\begin{tabular}{|r|c|}
\hline Name: & DMARS Defaults \\
\hline VarName: & ops_IMU2 \\
\hline Roll: & -1.25 \\
\hline Pitch: & 0.30 \\
\hline Yaw: & 0.00 \\
\hline Scanner: & 0.000 \\
\hline Range M: & 0.796 \\
\hline X offset: & -0.47 \\
\hline Y offset: & -1.40 \\
\hline Z offset: & -1.30 \\
\hline Chn1 range bias: & 0.00 \\
\hline Chn 2 Range bias: & 0.00 \\
\hline Chn3 Range bias: & 0.00 \\
\hline Max sfc sat: & 0 \\
\hline Dismiss & Use these \\
\hline
\end{tabular}

Figure 16. The ops_conf settings window.
There should be a different roll bias for each day, so a different ops_conf file should be placed in each mission day's folder. Review the following steps to determine the proper roll bias constant for the applicable mission day.

If not already completed, begin loading the data by repeating the steps in Opening ALPS Processing Windows, Loading the EAARL Database File, Loading GPS Flight Track Information, Loading DMARS Information, and Loading Mission Constants sections of this manual. Zoom in to a small area of the loaded flight lines in the Yorick 6 window. For determining the roll bias, the user should focus on areas where overlapping flight lines exist. Those flown over an airport runway or parking lot are ideal candidates for analysis. It is possible to analyze the data over features with variable slopes (sand dunes), but data from flat slopes will result in a more accurate determination of the roll bias. To find a flat area, the user may want to load the flight lines (. $\mathrm{kml} / \mathrm{kmz}$ files) in Google Earth.

If the Process EAARL Data window is not open, go to the eaarl window and select Lidar >> Process Lidar Data directory. Select an area of flight lines to process by going in the Define Region drop-down menu on the Process EAARL Data window. (See Topographic Lidar Data Processing for reference.) Select one of the following tools:

- Points in Polygon

- Rubberband Box

- Rectangular Coords

Once a tool is chosen, a window will open discussing the selection. If the Points in Polygon tool was selected, draw a polygon over the flight lines. If processing for sub-aerial topography elevations, select Topo Under Veg in the dropdown menu located next to the Define Region drop-down menu. Choose Submerged Topography if processing for bathymetric elevations. It is not necessary to use the Method drop-down menu or the in Win: menu. Click on Process Now and wait until the > appears in the command prompt, indicating that the processing has finished.

To view the processed data, select the Variable as veg_all or depth_all for sub-aerial topography or submerged topography, respectively. Select the Mode as Bare Earth Topography or Submerged Topography. Do not change in Win menu in the Process EAARL Data window. Click on the Auto Fma button to refresh the screen after each plot. Click the Histogram Elevations button and select the Both button to determine the maximum elevation, Cmax, and the minimum elevation, Cmin, to be displayed. Click Plot to display the processed data.

To evaluate the roll bias, use the mtransect command. This command will be used to view a two-dimensional slice of the elevation data by creating a graph of all of the points located within $1.5 \mathrm{~m}$ of the drawn line. Type in the command line info, mtransect or go to the CmdLine menu in the Process EAARL Data window and select mtransect. A window will 
open in which instructions for usage of the function and descriptions of function inputs are displayed. In the command line, type the following:

mtransect, variable to process, iwin=input, owin=output, xfma=1, rtn= [1-veg last return and 2-submerged topo], show $\mathbf{1}$ [press enter]. For example, mtransect, veg_all, iwin=5, owin=4, xfma=1, rtn=1, show=1 [press enter]. Use only $\mathbf{r t n}=\mathbf{1}$ and veg_all as the variable to process on last return topography data. Use $\mathbf{r t n}=\mathbf{2}$ and $\mathbf{d e p t h}$ _all as the variable to process when performing roll bias analysis on submerged topography data. The mtransect command is intended for last return or submerged topography data.

Draw a line in the input data window by left clicking the mouse and dragging across the plotted data. Release the mouse to view the output window, which displays elevation data from separate flight lines as different colors. In the output window, zoom in to the overlapping areas until the $y$-axis has $0.5-\mathrm{m}$ intervals. The difference in elevation between the flight lines should be $<0.5 \mathrm{~m}$ (fig. 17). Another problem that can be encountered is a dip down or a dip up in overlapping sections (this would appear as an X on the output screen). In this case, try lowering the elevation difference between the flight lines by changing the roll bias value. This can be done by typing ops_conf.roll_bias=\# [for example, ops_conf.roll_bias=-1.22] into the command line.

Every time a new roll bias is entered, the data will have to be reprocessed. Note that these changes are only temporary and will only be made permanent when the ops_conf file is changed by using editors such as gedit or kedit. To process and view the data with the new roll bias, repeat steps in this section. To determine if the new roll bias was a better fit for the data, reevaluate the elevation differences using the mtransect command in the same location as the previous mtransect command. Once the final roll bias is determined, make the final edit to the ops_conf file by typing kedit file name. [for example, kedit ops_conf_multi_base.i] in the command line. At this point, it is possible to batch process the data.

\section{Batch Processing Mode}

Batch processing is used to process the entire flight path for a given mission day. This process automatically sections the data into 2-kilometer $(\mathrm{km})$ by $2-\mathrm{km}$ data tiles. In the 2-km by $2-\mathrm{km}$ tiling scheme, data are tiled based on the UTM coordinates of the northwest corner. The $2-\mathrm{km}$ by $2-\mathrm{km}$ data tiles are further organized into $10-\mathrm{km}$ by $10-\mathrm{km}$ index tiles (fig. 18). These index tiles are also named based on the UTM coordinates of the northwest corner. Data tiles are named as $t \_$eXXX000_nXXXX000_XX. The $e X X X X 000$ is the easting of the northwest corner and the $n X X X X 000$ is the northing of the northwest corner. The attached XX to the file name is the UTM zone for the tile. Index tiles are named similarly but begin with "i" instead of "t", thus i_eXXX000_nXXXX000_XX (i_e451000_n4240000_18 for the index tile and $t \_e 454000$ n4242000_18 for the date tile). The batch processing mode

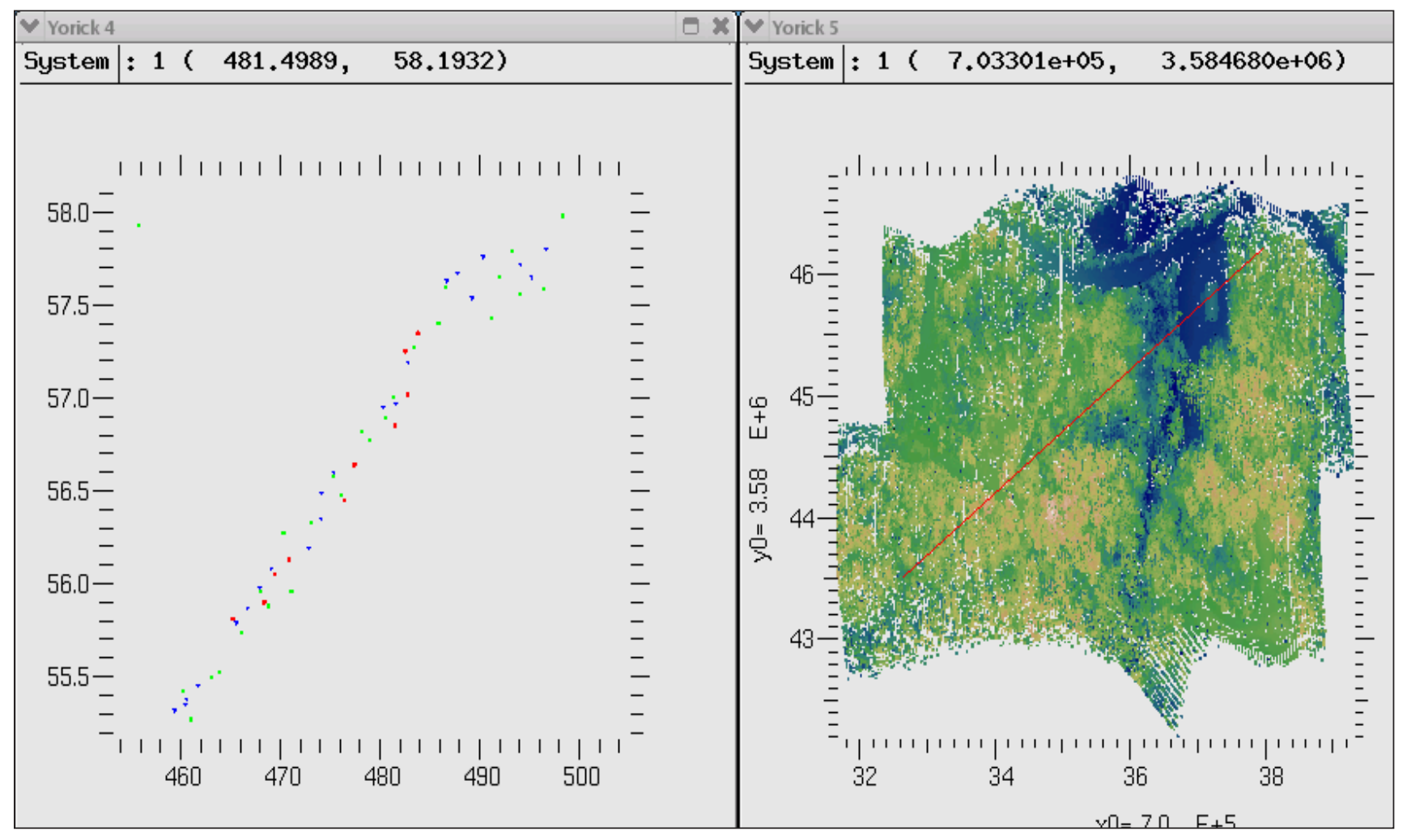

Figure 17. The panel on the right shows elevation data from overlapping flight lines. A vertical transect (represented by the red line) through the data is shown on the panel on the left. Each color represents a flight line. 
can only be performed after all of the mission data are loaded and the roll bias has been determined for the flight day via interactively processing small segments of the flight path. (See the Interactive Processing Mode sections.)

Begin the batch processing by typing help, batch process into the command line. Alternatively, select batch process within the CmdLine drop-down menu on the Process EAARL Data window. This will open a help document about what parameters to include in the batch processing command (table 6). Set the UTM zone number by typing curzone=\# [for example, curzone=16] in the command line. Once the parameters are determined, enter the following command in the command line: batch_process, typ=, save_dir="', zone=, mdate $="$ ", pick=, update $=$ [press enter $]$. For example, batch_ process, typ=1, save_dir="/training/katrina/Index_Tiles/", zone=16, mdate="20050908", pick=1 [press enter].

After the command, which includes pick=1, has been entered, the prompt will state the following: Hold the left mouse button down, select a region. If pick=2, the prompt will state: Left mouse generates a vertice. Ctl-Left or middle mouse click to end and close polygon. Go to the Yorick 6 window (displayed flight lines) and select the area to process.

Green boxes will appear over the flight lines; these represent the 2-km by $2-\mathrm{km}$ data tiles into which the data will be separated. The command window will run through the batch-processing sequence. The command window will list the details of processing, and the data tiles will turn black on the screen after the processing has finished (fig. 19). A return [>] will also appear at the command line. This process may take up to a day to complete, depending upon the amount of data processed. This, in turn, depends on the size of the selected area. It is best to initiate this process at the end of the day so that it will run overnight. The batch_process command only has to be executed only once per mission day; the user will have to batch process the data only once for first and last return topography. The separation of the data into bare earth and first return occurs during the filtering step.

\section{Filtering}

ALPS applies statistical filtering methods to remove false bottom returns and other outliers from the EAARL lidar data. Erroneous (outlier) points might include reflections from objects such as birds, multiple atmospheric effects (dust, moisture), or multiple reflections from bright targets. Two filtering methods within ALPS are used to extract ground (bare earth) elevations from a point cloud of processed last returns. These methods are the Random Consensus Filter (RCF) and the Iterative Random Consensus Filter (IRCF). The RCF is the faster and most basic filter in ALPS. The IRCF is a

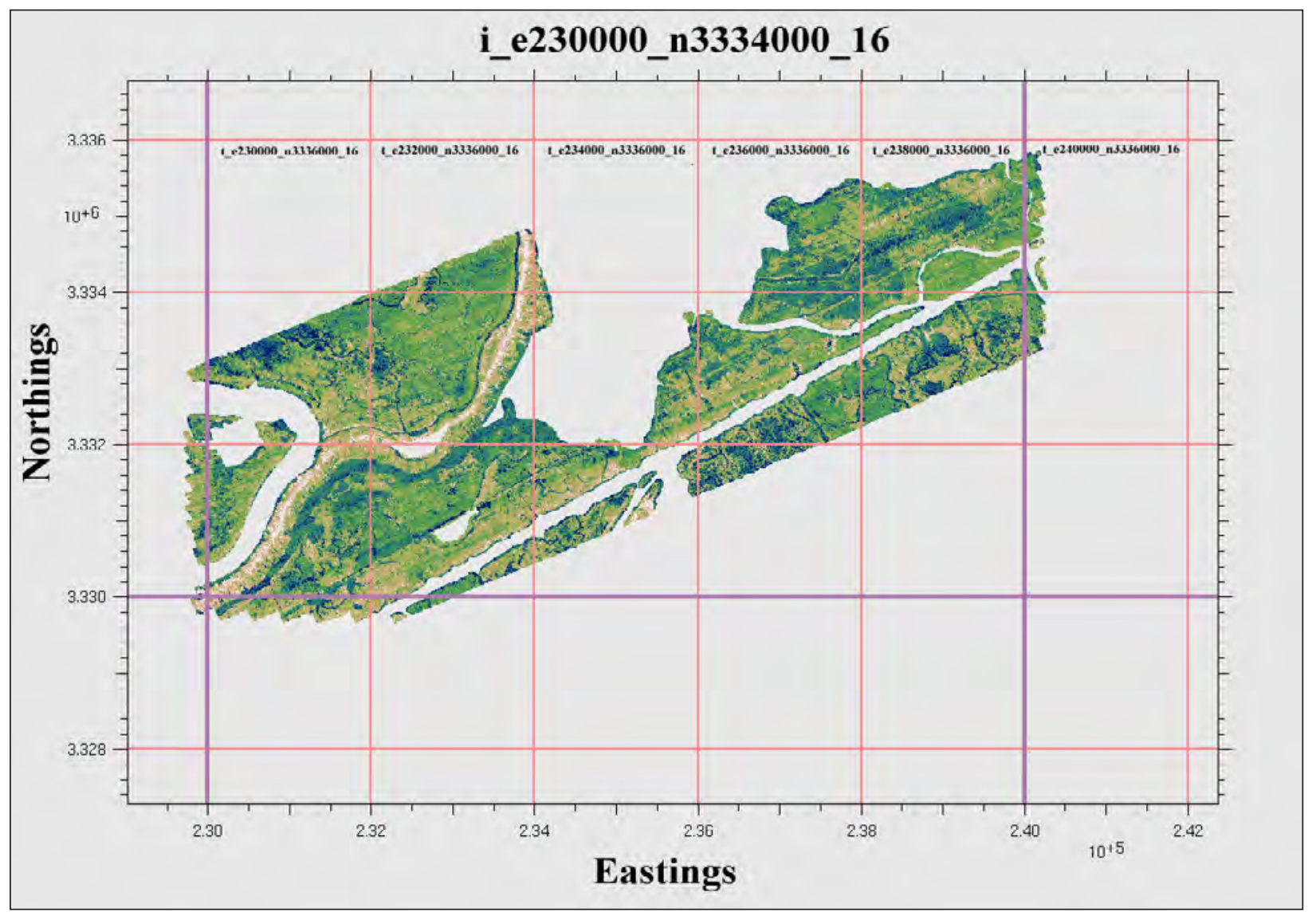

Figure 18. The 2-km by $2-\mathrm{km}$ data tiles are highlighted in pink and the $10-\mathrm{km}$ by $10-\mathrm{km}$ index tiles are highlighted in purple. 
Table 6. Batch processing parameters.

\begin{tabular}{|c|c|}
\hline Feature & Functionality \\
\hline typ & $\begin{array}{l}\text { Processes data type. For example, } 1 \text { for bathymetry } \\
\text { and } 2 \text { for vegetation (this includes first and last } \\
\text { returns). }\end{array}$ \\
\hline save_dir="" & $\begin{array}{l}\text { Where the files are to be written. Create an } \\
\text { Index_Tiles directory (enter in the command line: } \\
\text { mkdir Index_Tiles). The index and data tile } \\
\text { files will go inside of this directory [for example, } \\
\text { save_dir="/training/katrina/lndex_Tiles/"]. }\end{array}$ \\
\hline zone & Sets the UTM zone number [for example, zone=16] \\
\hline mdate="" & $\begin{array}{l}\text { Is the date the mission was flown in a yyyymmdd } \\
\text { format [mdate="20050908"]. }\end{array}$ \\
\hline onlyplot & $\begin{array}{l}\text { Set to } 1 \text { to plot data. This function will not process } \\
\text { data. This function will plot boxes around the } \\
\text { data tiles that are to be processed. }\end{array}$ \\
\hline pbd or edf & $\begin{array}{l}\text { Writes out the files to either a pbd (default) or .edf } \\
\text { format. Select only one output format. }\end{array}$ \\
\hline win & $\begin{array}{l}\text { Designates the location of the flight line } \\
\text { information. The default for this function is } \\
6 \text {. This will not be changed unless the users } \\
\text { changes the flight line window. }\end{array}$ \\
\hline pick & $\begin{array}{l}\text { Set equal to } 1 \text { to use the rubberband box selection } \\
\text { method or to } 2 \text { to use the points in a polygon } \\
\text { selection method. }\end{array}$ \\
\hline update & $\begin{array}{l}\text { Set to } 1 \text { to process files that have not been } \\
\text { processed. This function will not affect already } \\
\text { processed files. }\end{array}$ \\
\hline avg_surf & $\begin{array}{l}\text { Set to } 1 \text { to use the average water surface } \\
\text { reflections when processing for bathymetry. The } \\
\text { default for this parameter is avg_surf }=1 \text {. Set to } \\
0 \text { to disable this function. Set this variable to } 0 \\
\text { when the use Fresnel reflections to determine } \\
\text { water surface has been unselected. }\end{array}$ \\
\hline
\end{tabular}

slower process, because it combines several RCF passes with an iterative triangulation technique.

The RCF is based on the Random Sample Consensus (RANSAC) paradigm, which was originally published by Fischler and Bolles (1981). The filter uses a grid of nonoverlapping square cells of user-defined size overlaid onto the original point cloud. The user also defines the grid cell size and vertical tolerance based on the topographic complexity and point-sampling density of the data. The maximum allowable elevation range within a cell is established by the vertical tolerance (Nayegandhi and others, 2004). An iterative process searches for the maximum concentration of points within the vertical tolerance and removes those points outside of the tolerance (fig. 20).

For the IRCF, the RANSAC paradigm is used to determine the initial point cloud that represents the ground.

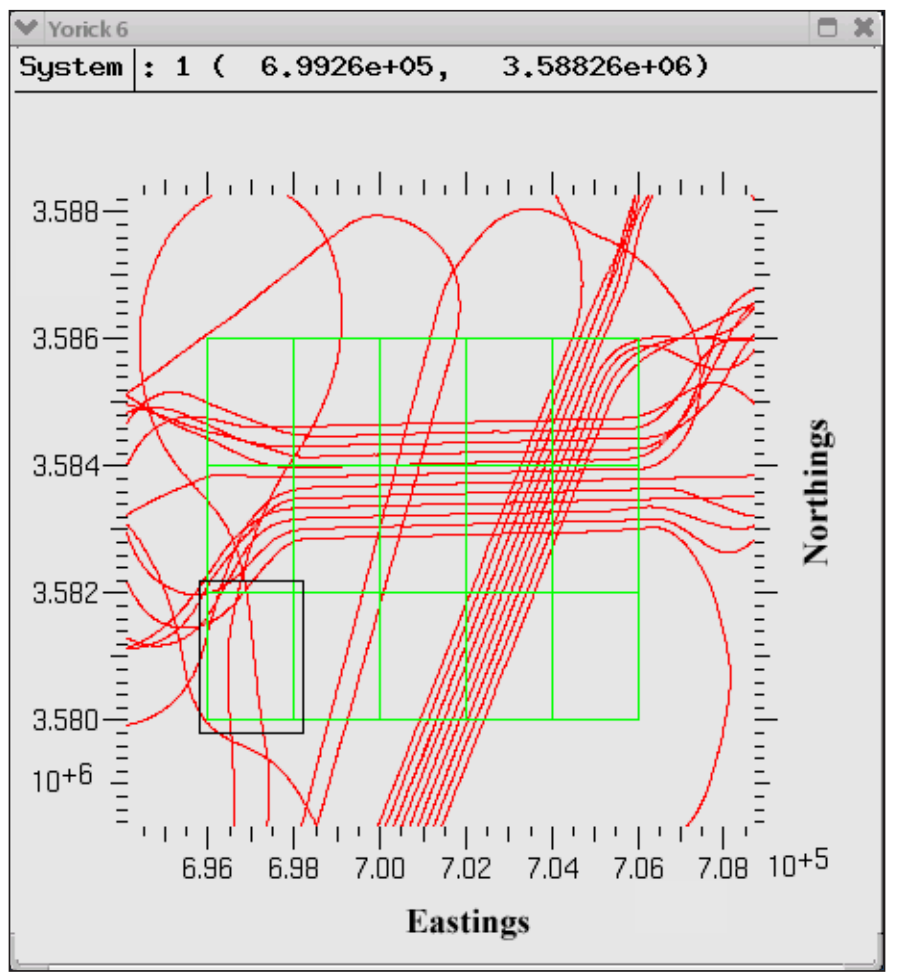

Figure 19. Highlighted in green are the flight lines selected to process in batch processing mode. As the flight lines are processed, they are highlighted in black.

A triangulated irregular network (TIN) model is created from the labeled ground points. The TIN model is continuously densified by adding all potential ground points within the vertical width for each triangulated facet. The points rejected from the first RANSAC iteration are treated as potential ground points. Each triangulated facet within the TIN model is defined as a three-dimensional plane, the equation of which is determined from the vertices of the triangulated facet. All potential ground points above or below each facet are classified as ground if they fall within the user-defined vertical range (also called the TIN elevation width) from the threedimensional plane. The process continues for a predefined number of iterations or when less than $2 \%$ of potential ground points are added to the final set of ground elevations (Nayegandhi and others, 2009).

\section{Random Consensus Filtering}

The first return topography data must be filtered using RCF, which can also be used to filter last returns and submerged topography returns where appropriate. The RCF function can be used to test the filter parameters on the dataset or to refilter a specific portion of the dataset after a batch filter has been applied. (See Manual Filtering/Editing section.) The following filtering protocol can be performed only after each mission has been batch processed. See the previous section on Batch Processing Mode for completing these steps. 


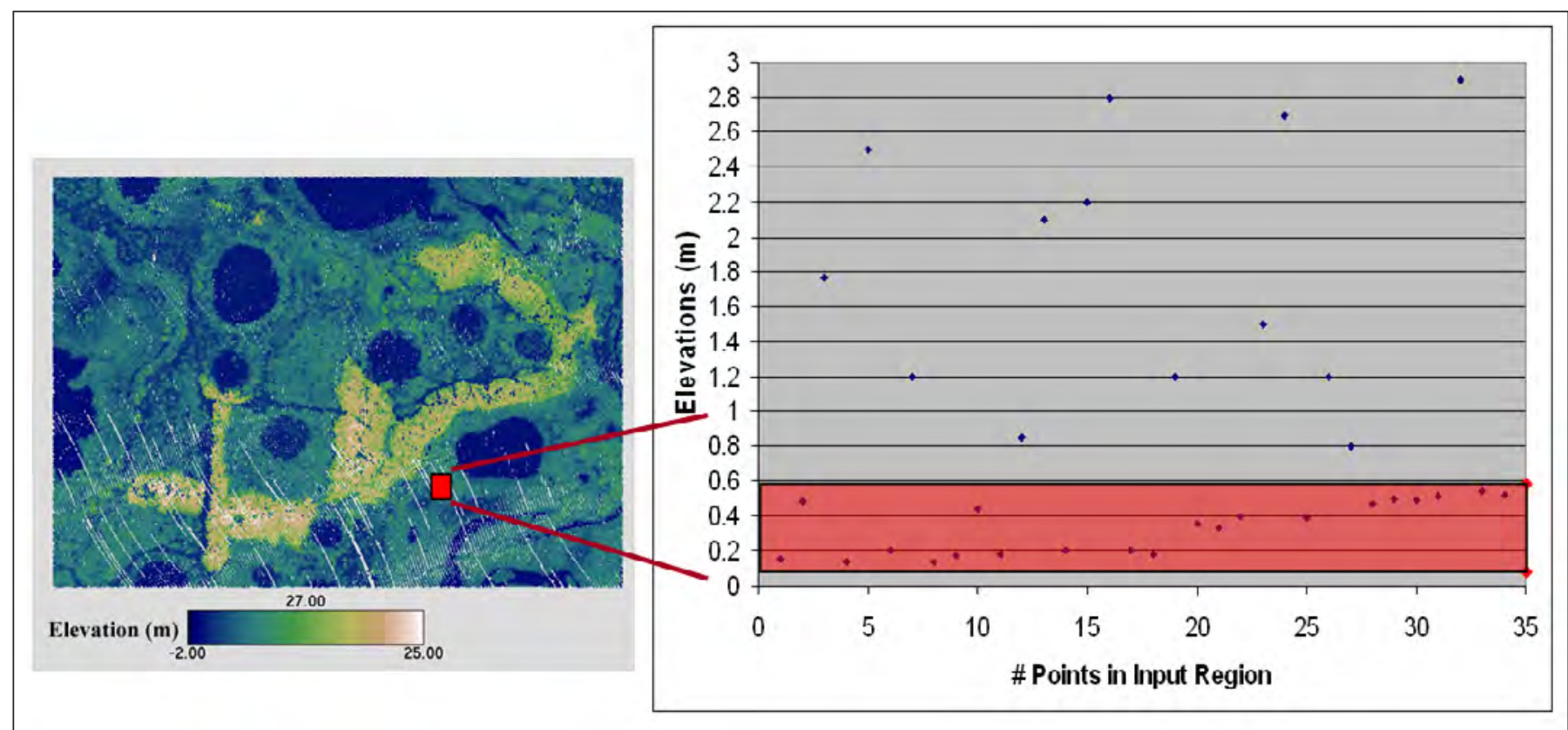

Figure 20. The image on the right shows a vertical slice of data from the image on the left. The image on the right shows a concentration of points on the ground and several outliers. The data outside of the red rectangle highlight the points that would be removed (Nayegandhi and others, 2004).

\section{Interactive RCF}

Go to the PostProcessing menu in the Process EAARL Data window and select RCF Region Filter. The Random Consensus Filter window will open (fig. 21). In the Select RCF type drop-down menu, select RCF. The Input Window $(\mathrm{cm})$ default value is 500 centimeter $(\mathrm{cm})$ and the Elevation width $(\mathrm{cm})$ default value is $20 \mathrm{~cm}$. For the buffer [Input Window], choose a value around 600 or higher. This value would partition all of the points into 6-m by 6-m squares. This value should decrease to account for increasing sloping regions. The input window also depends on the data density. Select a smaller window size if the density is high. The tolerance or vertical window [Elevation Width] should be a value of around 400 or higher for first return topography. If using this RCF for last return topography, the value should be around 20 to 40 $\mathrm{cm}$ for relatively flat surfaces, and 40 to $100 \mathrm{~cm}$ for sloping surfaces. If the analyst has prior knowledge of the mapped region, select the tolerance value to be the maximum range of elevation expected within the input window. Set Minimum

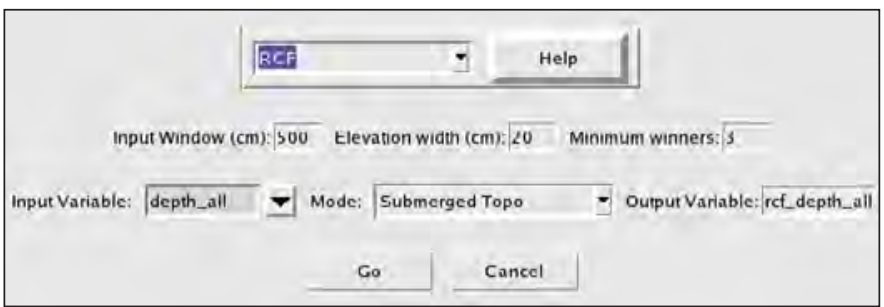

Figure 21. The Random Consensus Filter window. winners to 3. The Input Variable takes the loaded variable from the Process EAARL Data window. Make sure the correct tile is being filtered. The Mode should be changed to whatever mode is being viewed. This Mode will be the same as the Mode on the Process EAARL Data menu. The Output Variable will already be created and $r c f_{-}$will be inserted to the beginning of the Input Variable name [for example, Input Variable: bet_20070912_6 and Output Variable: rcf_bet_20070912_6]. Click the Go button; the RCF function will start to process. The command terminal will display a return $[>]$ when the filtering has been completed.

To view the filtered data, go to the Process EAARL Data window and choose a different in Win: for the data to display. Select the new variable [for example, rcf_bet_20070912_6] by clicking the Variable drop-down menu and click Plot to view the newly filtered data. If these are the desired parameters with which to RCF filter the data, then run the batch filter for the remaining data. (See Batch Filtering section of this manual for instructions.)

\section{Iterative Random Consensus Filtering}

The IRCF is used to filter last returns and submerged topography returns. If utilized in the interactive mode, IRCF can be used manually to edit erroneous elevations in the dataset by deleting triangles from the TIN surface. This meticulous process focuses on the final elevation points consistent with bare earth and submerged topography.

The IRCF parameters (fig. 22), without the interactive functions, are similar to those used with the RCF function. The 
only difference is that additional parameters such as minimum and maximum Pre-filter Elevations $(\mathrm{m})$, TIN elev width $(\mathrm{cm})$, Plot TIN in win, and the Distance Threshold $(\mathrm{m})$ can be set or changed to affect the iterative process. Input Window should be a value of about $500 \mathrm{~cm}$ or higher. The Elevation width $(\mathrm{cm})$ should be about 20 to $40 \mathrm{~cm}$ for flat surfaces and 30 to $80 \mathrm{~cm}$ for sloping surfaces. Submerged topography should be treated similar to last return topography when determining filtering parameters.

From the Process EAARL Data window, select Random Consensus Filter and select Iterative RCF from the dropdown menu (fig. 22). The minimum and maximum Pre-filter Elevations $(\mathrm{m})$ should be set if the analyst is familiar with the elevation range within that study area. The Tin elev width $(\mathrm{cm})$ should be set to 20 to $30 \mathrm{~cm}$ for relatively flat areas and 20 to $60 \mathrm{~cm}$ for sloping areas. Set Minimum winners as 3. The Input Variable takes the loaded variable from the Process EAARL Data window. The Mode should be changed to whatever mode is being viewed. The Output Variable will already be created and $r c f_{-}$will be inserted into the beginning of the Input Variable name. Click the Go button; the filter will begin processing. The command terminal will display a return $[>]$ when the processing has been completed.

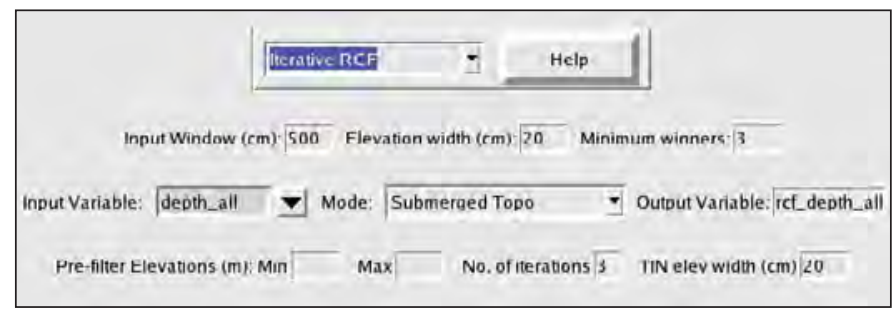

Figure 22. The Iterative Random Consensus Filter window.

\section{Interactive IRCF}

To use the Interactive IRCF, repeat steps from the IRCF section above. Click the Interactive? button and click Go. The IRCF filter will run and a new window will open with gridded data. Zoom in on the triangles with erroneous data that need to be deleted. The command prompt will read Interactive Mode? (yes/no/done/end). Enter yes in the command line. Click left to continue selection, middle to pan/zoom, right to select similar; CTRL-Right=End Interactive Mode or CTRL-left=Retriangulate. Select similar triangles to delete, then control-left to retriangulate the area. The prompt will then ask to continue interactive mode. Typing no or done on the command line will put the area through a series of iterations while allowing the user to select triangles for further deletion. Continue repeating the above steps until the entire area is ready to be retriangulated; type end to finish the iterations.

To view the filtered data, go to the Process EAARL Data window and choose the filtered data to plot. The new variable should already be loaded in the list. Select the $r c f_{-}$variable [for example, rcf_bet_20070912_6] and click Plot to view the newly processed data. If these are the desired parameters with which to IRCF filter the data, then run the batch filter for the remaining data. (See Batch Filtering section of this manual for instructions.)

\section{Batch Filtering}

Like batch processing, batch filtering saves valuable time by eliminating the effort required to interactively filter the lidar data. Additional erroneous data can be removed during the manual editing session after batch filtering has been completed. (See Manual Filtering/Editing section.)

If the windows: ytk, eaarl, and Process EAARL Data are not currently open, see the Opening ALPS Processing Windows section for instruction. Opening these windows will load a series of files that are necessary for batch filtering. The user need not load the day of flight data [pnav, dmars, or ops_conf files] that were required for the interactive and batch processing detailed in the Processing of Raw EAARL Data section of this manual.

In the command line, type help, batch_rcf or select from the Process EAARL Data window CmdLine >> batch_rcf(Filter). A window will open in which instructions for usage of the batch filtering function and descriptions of function inputs are displayed (table 7). Set the UTM zone number by typing curzone=\# [for example, curzone $=\mathbf{1 6}$ ] in the command line. Once the user has determined the parameters needed to batch filter, type the following command:

batch_rcf, "directory", buf=, w=, mode=, meta=, write merge $=$, rcfmode $=$, searchstr=" " [press enter]. For example,

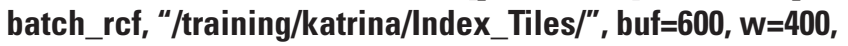
mode $=1$, meta $=1$, write_merge $=1$, rcfmode $=1$, searchstr $={ }^{*}$ _v. pbd" [press enter].

This batch filtering process can take anywhere from a few hours for the RCF to a couple of days for the IRCF; it also depends on the amount of data that are being filtered. Once the function has finished, the user will see a return $[>]$ at the command prompt.

\section{Image Plots}

ALPS includes utilities to generate image plots with the processed and filtered EAARL data. Viewing the plots confirms that the appropriate mission constants and filter parameters were applied for the survey area.

\section{Loading Image Plot Data}

If the windows ytk, eaarl, and Process EAARL Data are not currently open, see the Opening ALPS Processing Windows section for instruction. To load data for image generation, select the Read Data Directory option within the File menu in the Process EAARL Data window. The Read Data 
Table 7. Batch filtering parameters.

\begin{tabular}{|c|c|}
\hline Feature & Functionality \\
\hline "directory" & $\begin{array}{l}\text { Is the directory name within which all the files are } \\
\text { processed [for example, "/training/katrina/ } \\
\text { Index_Tiles/"]. }\end{array}$ \\
\hline buf & $\begin{array}{l}\text { Is the horizontal size in centimeters of the area to } \\
\text { be filtered (default is } 700 \mathrm{~cm} \text { ). }\end{array}$ \\
\hline w & $\begin{array}{l}\text { Is the vertical height in centimeters (default is } 200 \\
\text { (cm). }\end{array}$ \\
\hline mode & $\begin{array}{l}\text { The type of data to be filtered. Set to } 1 \text { for first } \\
\text { return, } 2 \text { for submerged topography, and } 3 \text { for } \\
\text { ground under vegetation (bare earth). }\end{array}$ \\
\hline meta & $\begin{array}{l}\text { Set to } 1 \text { for the RCF parameters to include the file } \\
\text { name. }\end{array}$ \\
\hline write_merge & $\begin{array}{l}\text { Set to } 1 \text { to have the files merged before filtering. } \\
\text { This parameter is useful when using the manual } \\
\text { editing replace function. See the section on } \\
\text { Manual Filtering/Editing for details on the } \\
\text { replace function. }\end{array}$ \\
\hline rcfmode & $\begin{array}{l}\text { Sets the type of filter being applied to data. Set } \\
\text { to a value of } 2 \text { for the iterative RCF (IRCF filter } \\
\text { for bare earth and submerged topography only), } \\
1 \text { for the plain RCF filter, and } 0 \text { to disable the } \\
\text { filter (use when doing a merge only). The default } \\
\text { value for this parameter is } 2 \text {. }\end{array}$ \\
\hline searchstr="" & $\begin{array}{l}\text { Searches for these files to process [for example, } \\
\text { searchstr="*_v.pbd"]. }\end{array}$ \\
\hline update & $\begin{array}{l}\text { Set this to } 1 \text { to process only those files that have } \\
\text { not yet been filtered. This will not affect already } \\
\text { filtered files. }\end{array}$ \\
\hline
\end{tabular}

Directory window will open. In the window, click the Browse button to navigate to the directory with the index tiles. Select one of the index tiles that includes data. In Data Type, select .pbd. Search String defines the type of files to be loaded. Type *_v.pbd to load processed, unfiltered last return and first return data, ${ }^{*}$.b.pbd to load processed, unfiltered submerged topography data, and ${ }^{*} \mathbf{f s}^{*}{ }^{*} \mathbf{c f}^{*}$.pbd as an example to load the first return processed, filtered data. In the Merged Variable, specify a variable name for the data. Use a variable name that will be easy to remember and that reflects that type of data loaded [for example, fs_rcf_merge]. Click the Unique button to load only one of the overlapping points from each data tile. Choose a Subsample number. A number between 10 and 50 is a good range of values for viewing 2 to 10 data tiles, but the numbers will depend on the size of the area being plotted and the point density. The data may overlap UTM zones. To view a particular UTM zone, click on the Fixed Zone button. Select the Zone number and select whether the data is in $2 k$ Data Tiles or Quarter Quads. (See Quarter Quadrangle Description section.) Click Load Data.

\section{Viewing Image Plot Data}

To view the loaded data, make sure the variable with the loaded data was added to the variable list. Click the Variable button on the Process EAARL Data window. This will open the List window. In the List window, click on the merged variable name in the list and click the Select button, or go to the Process EAARL Data window and use the Variable dropdown menu to select the merged variable name. If the variable name is not in the list, enter it into the List window and click Add; click the Select button to select the variable. Confirm the Mode is appropriate whether it is the First Return Topography, Bare Earth Topography, or Submerged Topography. Change in Win: to another number in order to have this data plot in another window. Select the Histogram Elevations button to determine the maximum elevation, Cmax, and the minimum elevation, Cmin, to display. To change the graph size, go to the Graph menu in the Process Eaarl Data window and select the $100 \mathrm{Dpi}(1100 \times 850)$ to be able to plot the largest size graph. Always confirm that the Auto Fma button is depressed to refresh the plot with subsequent changes made to the graph. Click Plot. Repeat steps for other types of data.

\section{Saving Image Plots}

Finally, save an image of the plotted data (figs. 23 and 24). First make sure the image to be saved is the current window specified in in Win: in the Process Eaarl Data window. Next, add a color bar, which defines the minimum and maximum elevation values shown in the plot. Click the Color Bar button. Left click and drag into the window with the loaded data. Select an area where the color bar will be displayed. Create a plot title by typing pltitle ("Title" ) on the command line. The title will appear at the top of the loaded data window (figs. 23 and 24); pltitle ("Before IRCF").

To save this image, go to the Imagery menu in the eaarl window and select Capture a display. A window will open with the following instructions: Raise the window you want to capture, click $O K$, and then click on the desired window. Click $O K$ on the instructional window and click on the window to be captured. A Save As window will open. Navigate to an appropriate directory in which to save the image and click Save. This will automatically save the image as a .png file.

\section{Datum Conversion}

ALPS includes utilities for projecting the data to other datums so that the dataset can be compared with other existing datasets. Geodetic datums represent the size and shape of the Earth and the origin and orientation of the coordinate systems. Datums have evolved over time from those describing a spherical Earth to ellipsoidal models derived from satellite measurements. Datum changes require a periodic update due 


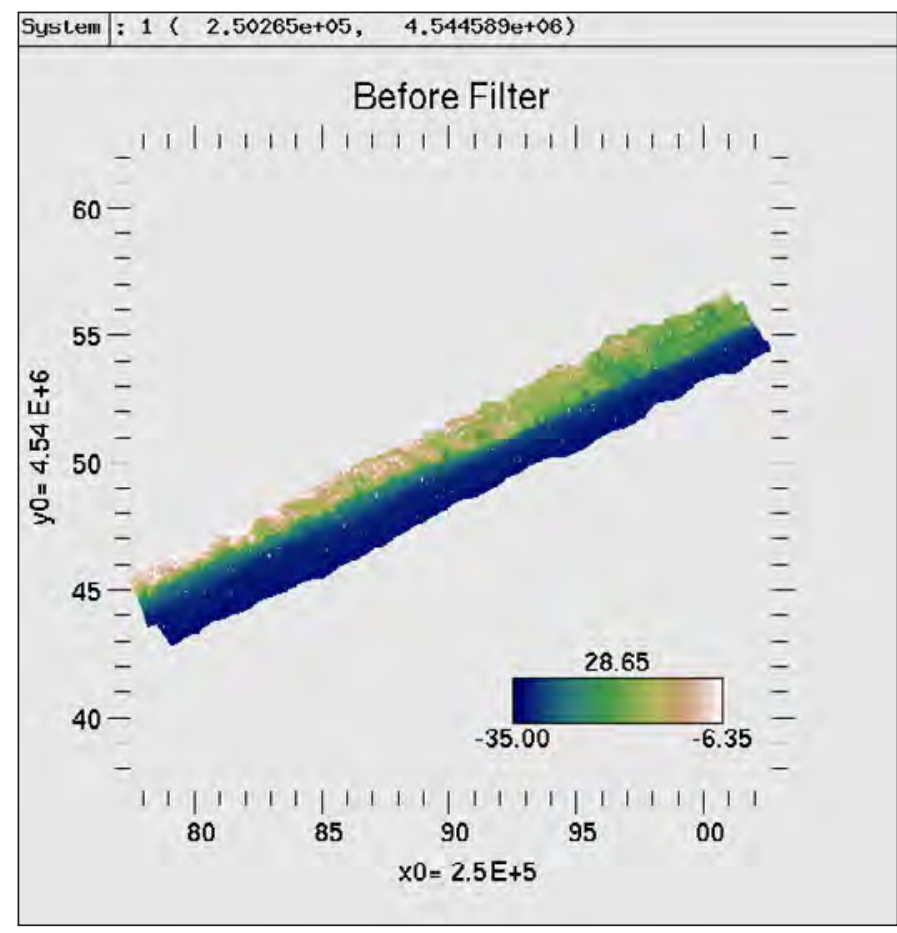

Figure 23. Image plot showing unfiltered elevation data from $-35 \mathrm{~m}$ to $-6.35 \mathrm{~m}$. Elevation values are in WGS84 ellipsoid heights. The yellow specks represent noise that filtering would help remove.

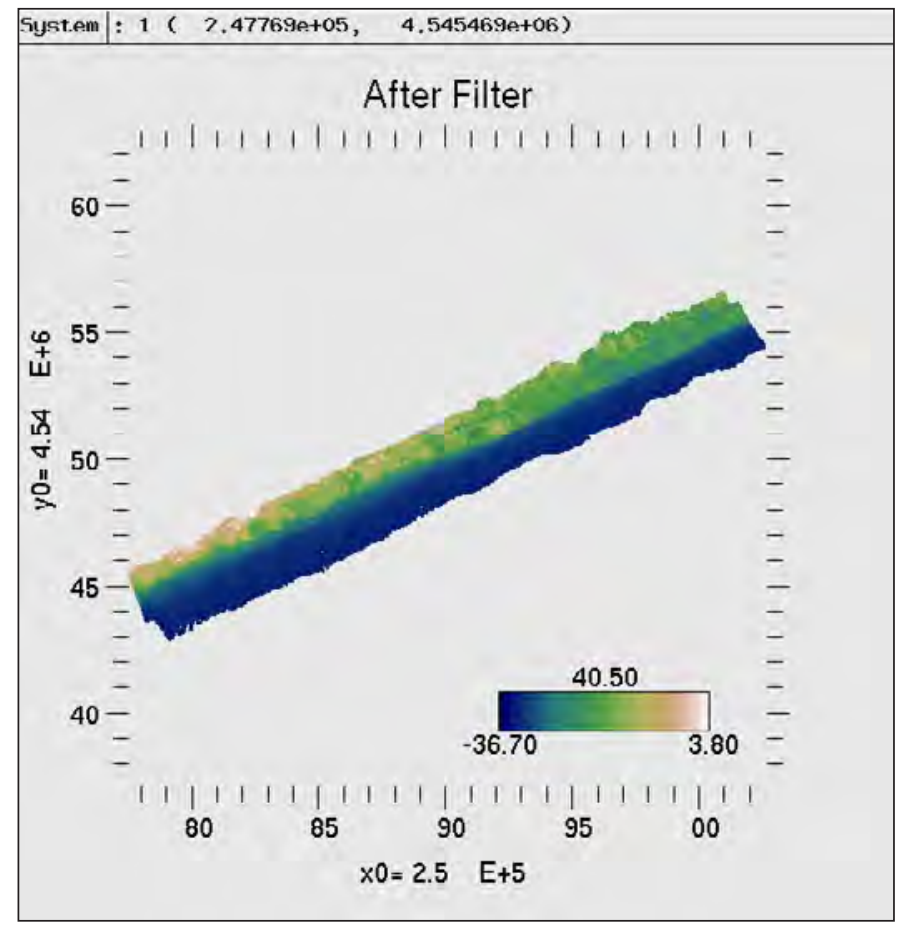

Figure 24. Image plot showing filtered elevation data from $-36.70 \mathrm{~m}$ to $-3.80 \mathrm{~m}$. Elevations values are in WGS84 ellipsoid heights. The yellow specks have mostly been filtered out. to changes in the geologic structure of the Earth's surface, such as subsidence, uplift, and sea-level change. ALPS can convert the ellipsoid heights (WGS84 G1150) to orthometric heights (NAD83, NAVD88) using the GEOID03 model. This is an important process because each datum transformation can introduce a 1- to 5-cm error into the data with each datum transformation (Nayegandhi and others, 2006).

Datum conversions occur in the batch mode and the process generates a new . pbd file with_n88 in the file name. If the windows ytk, eaarl, and Process EAARL Data are not currently open, see the Opening ALPS Processing Windows section for instructions. Opening these windows will load a series of files that are necessary for batch datum conversion to begin. Set the curzone/utmzone [for example, curzone=16 or utmzone=16]. Type help, batch_datum_convert in the command line. Alternatively, select batch_datum_convert within the CmdLine menu in the Process EAARL Data window. This will open a help document about the parameters to include in the batch datum conversion command (table 8). Type the following command: batch_datum_convert, "directory", zone_nbr=, searchstr="' [press enter]. For example, batch_datum_convert, “/training/katrina/Index_ Tiles/", zone_nbr=16, searchstr="*merged_rcf.pbd" [press enter].

Datum conversions can take a couple of hours or less to complete, depending on the data size. Once the function has finished, a return [>] will appear in the command prompt.

Table 8. Batch datum conversion parameters.

\begin{tabular}{|l|l|}
\hline \multicolumn{1}{|c|}{ Feature } & \multicolumn{1}{|c|}{ Functionality } \\
\hline "directory" & $\begin{array}{l}\text { The directory name within which the files are } \\
\text { processed [for example, "/training/katrina/ } \\
\text { Index_Tiles/"]. }\end{array}$ \\
\hline zone_nbr & Sets the UTM zone number. \\
\hline searchstr="" & $\begin{array}{l}\text { Sets the search string instead of using rcfmode } \\
\text { [for example, searchstr="*ircf*.pbd"]. }\end{array}$ \\
\hline tonad83 & $\begin{array}{l}\text { Set to } 0 \text { to not use this conversion. Otherwise } \\
\text { converts files to NAD 83 reference datum. }\end{array}$ \\
\hline tonavd88 & $\begin{array}{l}\text { Set to 0 if the user does not want to utilize this } \\
\text { command. Otherwise converts files to NAVD 88 } \\
\text { reference datum. }\end{array}$ \\
\hline rcfmode & $\begin{array}{l}\text { Set to 1 to convert RCF'd files and set to 2 to } \\
\text { convert IRCF'd files. This option is replaced by } \\
\text { using the search string. }\end{array}$ \\
\hline geoid_version & $\begin{array}{l}\text { Set to "GEOID99" to use the GEOID99 model } \\
\text { or "GEOID96" to use the GEOID96 model. } \\
\text { Otherwise, the default is the GEOID03 model. }\end{array}$ \\
\hline update & $\begin{array}{l}\text { Set to } 1 \text { to process only those files that have not } \\
\text { yet been converted. This will not affect already } \\
\text { converted files. }\end{array}$ \\
\hline
\end{tabular}




\section{Quarter Quadrangle}

The quarter quadrangle (QQ) tiling scheme is commonly used for organizing digital orthoimagery. Airborne Topographic Mapper (ATM) (Airborne Topographic Mapper Data Processing section) or some sizeable EAARL datasets are organized in this way. This tiling scheme is used for lidar data dissemination by the U.S. Geological Survey (USGS) Center for Lidar Information Coordination and Knowledge (CLICK). Furthermore, this tiling scheme facilitates lidar and orthoimagery comparisons, which can be an important component of lidar quality assurance and control.

\section{Quarter Quadrangle Description}

In the QQ tiling scheme, each tile is $1 / 16$ of a degree (3.75 minutes) in width and height. The tiles are named by referencing their southeast corners, with boundaries on every 1/16 of a degree latitude and longitude of the NAD83 datum. The naming scheme uses a structure of AAOOOOaoq (table 9). For example, $47104 \mathrm{~h} 2 \mathrm{c}$ means:

$47 \quad 47$ degrees north latitude

104104 degrees west longitude

$\mathrm{h} \quad$ is 8 th in sequence, so it is the last section and starts at $7 / 8$ of a degree, or 0.875

22 is second in sequence, so it is in the 2 nd section and would start at $1 / 8$ of a degree, or 0.125

c this designates the northwest corner, which means we add $1 / 16$ of a degree to both the north and west, or 0.0625 to each.

47104 is the square degree designated at $47^{\circ} \mathrm{N}$., $104^{\circ}$ $\mathrm{W}$. The $\mathrm{h} 2$ describes the quadrangle with a southeast corner at $47.875^{\circ} \mathrm{N} ., 104.125^{\circ} \mathrm{W}$. The $\mathrm{c}$ indicates it is the QQ with a southeast corner at $47.9375^{\circ} \mathrm{N}$., $104.1875^{\circ} \mathrm{W}$.

\section{ALPS 2-km to Quarter Quadrangle Conversion}

To convert ATM (Airborne Topographic Mapper Data Processing section) or EAARL data into the QQ format, type help, batch_2k_to_qq in the command line. This opens a help document about parameters to include in the batch QQ conversion (table 10). Type in the command line in this specific order: batch_2k_to_qq, "src_dir", "dest_dir", mode, searchstr="', dir_struc=1, buffer=30 [press enter]. For example: batch_2k_to_qq, "/training/katrina/Index_Tiles/", "/training/katrina/be_00_tiles/", 3, searchstr $={ }^{\text {"* }} \mathbf{n 8 8}{ }^{*}$ ircf ${ }^{*}$ mf.pbd", dir_struc=1, buffer=30 [press enter].

\section{Quarter Quadrangle to ALPS 2-km Conversion}

To convert the ATM (Airborne Topographic Mapper Data Processing section) or EAARL data into the 2-km format, type in the command line: help, batch_qq_to_2k. This opens
Table 9. Quarter quadrangle tiling scheme.

\begin{tabular}{|c|c|c|}
\hline $\begin{array}{c}\text { Character } \\
\text { Representation }\end{array}$ & Character Type & Designation \\
\hline AA & $\begin{array}{l}\text { Positive whole } \\
\text { number. }\end{array}$ & Degrees latitude. \\
\hline 000 & $\begin{array}{l}\text { Positive whole } \\
\text { number. Value } \\
\text { is zero padded } \\
\text { to a width of } \\
\text { three numbers, if } \\
\text { necessary. }\end{array}$ & Degrees longitude. \\
\hline a & Alpha character. & $\begin{array}{l}\text { Characters a-h } \\
\text { designate which } \\
\text { quadrangle in the } \\
\text { degree of latitude, } \\
\text { where a is the } \\
\text { closest to zero } \\
\text { minutes and } h \text { is the } \\
\text { closest to the next } \\
\text { full degree. Each } \\
\text { character represents } \\
\text { 1/8th of a degree. }\end{array}$ \\
\hline 0 & Numeral & $\begin{array}{l}\text { Numbers } 1-8 \text { designate } \\
\text { the quadrangle in the } \\
\text { degree of longitude, } \\
\text { where } 1 \text { is closest to } \\
\text { zero minutes and } 8 \\
\text { is closest to the next } \\
\text { full degree. Each } \\
\text { numeral represents } \\
1 / 8 \text { of a degree. }\end{array}$ \\
\hline$q$ & Alpha character. & $\begin{array}{l}\text { Characters a-d } \\
\text { designate the } \\
\text { quarter in the } \\
\text { quadrangle, where } \\
\text { a is the southeast } \\
\text { quarter, b is the } \\
\text { northeast corner, } \\
\mathrm{c} \text { is the northwest } \\
\text { corner, and d is } \\
\text { the southwest } \\
\text { corner. Each quarter } \\
\text { quadrant is } 1 / 16 \text { of } \\
\text { a degree in latitude } \\
\text { and } 1 / 16 \text { of a degree } \\
\text { in longitude. }\end{array}$ \\
\hline
\end{tabular}

a help document regarding which parameters to include in the batch conversion (table 11). Type in the command line in this specific order: batch_qq_to_2k, "src_dir", "dest_dir", mode, searchstr="", buffer=30 [press enter]. For example: batch_ qq_to_2k, “/training/katrina/Index_Tiles/", “/training/katrina/ be_00_tiles/", 3, searchstr="*n88*ircf*_mf.pbd", buffer=30 [press enter]. 
Table 10. Quarter Quadrangle to ALPS 2-km conversion parameters.

\begin{tabular}{|l|l|}
\hline \multicolumn{1}{|c|}{ Feature } & \multicolumn{1}{c|}{ Functionality } \\
\hline "src_dir" & $\begin{array}{l}\text { Designates the location of the source directory. It } \\
\text { should be the directory structure containing the } \\
\text { EAARL 2-km x 2-km tiles in .pbd format. }\end{array}$ \\
\hline "dest_dir" & $\begin{array}{l}\text { Designates the directory that the quarter } \\
\text { quadrangle .pbd would go into. }\end{array}$ \\
\hline mode & $\begin{array}{l}\text { The type of EAARL that will be converted. Use } \\
\text { only the number to represent the type of data. } \\
\text { Set the values equal to 1 for first return, 2 for } \\
\text { bathymetry, and 3 for bare earth. }\end{array}$ \\
\hline searchstr="" & $\begin{array}{l}\text { Searches for these files to process [for example, } \\
\text { searchstr="*n88*ircf*_mf.pbd"]. }\end{array}$ \\
\hline dir_struc & $\begin{array}{l}\text { Set equal to 1 to create a quarter quadrangle } \\
\text { directory structure similar to the index tile } \\
\text { structure. }\end{array}$ \\
\hline
\end{tabular}

\section{Airborne Topographic Mapper Data Processing}

The ATM is a single-return, green-wavelength lidar system developed by the National Aeronautic and Space Administration (NASA). The original purpose for this lidar system was to measure the changing Arctic and Antarctic ice caps and glaciers. The ATM has also been employed in observing coastal-morphology change. The ATM system is mounted to a small aircraft that flies around 400 to $800 \mathrm{~m}$ AGL (fig. 25). Tested accuracy of the ATM data is about 10 to $20 \mathrm{~cm}$. In 2001, a down-looking camera was added to the ATM lidar system (Harris and others, 2005).

The ATM raw data are stored as binary .qi files and contain only first returns. All of the .qi files that are intended

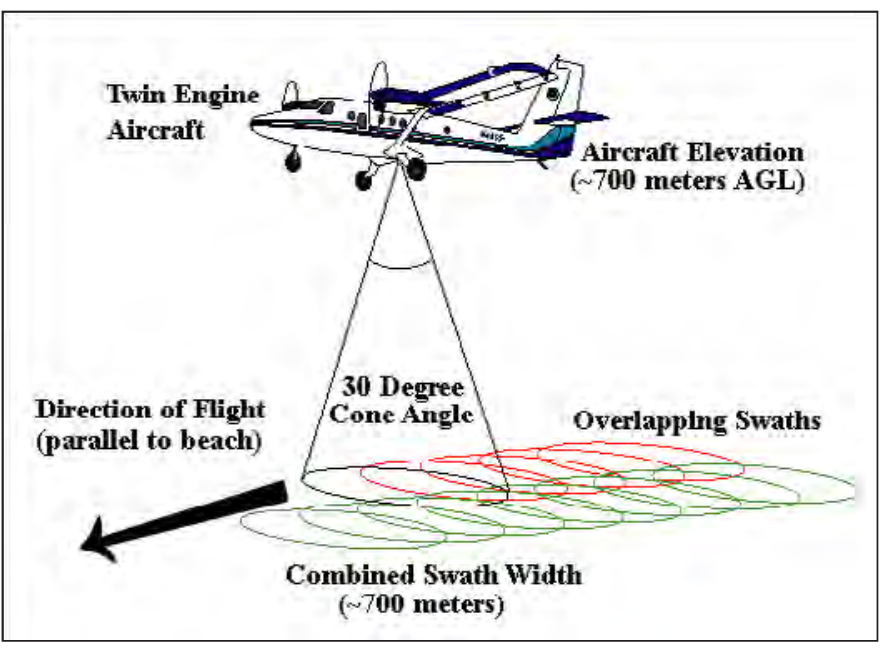

Figure 25. The elliptical scan pattern of the ATM system (Sallenger and Brock, 2001).
Table 11. Quarter Quadrangle to ALPS 2-km conversion parameters.

\begin{tabular}{|c|c|}
\hline Feature & Functionality \\
\hline "src_dir" & $\begin{array}{l}\text { The source directory [for example, "/training/ } \\
\text { katrina/be_qq/"]. This should be the root } \\
\text { directory that contains the } 00 \text { tiles in .pbd } \\
\text { format. }\end{array}$ \\
\hline "dest_dir" & $\begin{array}{l}\text { The destination directory. The index tiles can be } \\
\text { written here. }\end{array}$ \\
\hline mode & $\begin{array}{l}\text { Type of data converted. Use only the number } \\
\text { to represent the type of data. Use } 1 \text { for first } \\
\text { return, } 2 \text { for bathymetry, and } 3 \text { for bare earth. }\end{array}$ \\
\hline searchstr="" & $\begin{array}{l}\text { Searches for these files to process [for example, } \\
\left.\text { searchstr="* } \mathbf{q q}^{*} \cdot \mathbf{p b} \mathbf{p} \mathbf{d}^{\prime}\right] \text {. }\end{array}$ \\
\hline suffix & $\begin{array}{l}\text { Inserts a string at the end of each data tile file } \\
\text { name. By default, two letters will be inserted } \\
\text { based on the mode type. This can include } \\
\text { a trailing .pbd. To suppress the suffix, use } \\
\text { suffix="'.. }\end{array}$ \\
\hline remove_buffers & $\begin{array}{l}\text { Set to } 1 \text { to clip each } 00 \text {.pbd data to the file's } 00 \\
\text { extent. Set to } 0 \text { for all of the data to be used, } \\
\text { regardless of location. This function defaults } \\
\text { to } 1 .\end{array}$ \\
\hline buffer & $\begin{array}{l}\text { Specifies a buffer in meters added around each } \\
\text { data tile. Default is buffer=100. Use buffer=0 } \\
\text { to suppress the buffer. }\end{array}$ \\
\hline uniq & $\begin{array}{l}\text { Specifies whether data points should be } \\
\text { constrained to only unique points by sod when } \\
\text { saved to the pbd file. Default is } 1 . \text { Set equal } \\
\text { to } 0 \text { to avoid this constraint. This is necessary } \\
\text { with Airborne Topographic Mapper (ATM) } \\
\text { data, which may have unreliable sod values. }\end{array}$ \\
\hline
\end{tabular}

for processing should be separated by mission date. Multiple mission dates should not be stored in the same directory. Before beginning the ATM processing, open the following windows: ytk, eaarl, and Process EAARL Data. (See the Opening ALPS Processing Windows section for instruction.) conv Opening these windows will load a series of files required for the ATM processing command line scripts. Type help, batch_qi_to_tiles in the command line. This will open a help document explaining the parameters to include in the ATM processing command (table 12). The ATM data will be converted into the same index tile and data tile format as is done with the EAARL data. To process the data, type the following command: batch_qi_to_tiles, "con_dir", ymd, "dir", searchstr="*.qi", [press enter]. For example:

batch_qi_to_tiles,

"/training/qi_data/20011012/", 20011012,

"/training/Index_Tiles/", searchstr="*.qi" [press enter].

Next, merge the data tiles from each mission day by using the merge_qi_tiles function. Type help, merge_qi_tiles in the 
Table 12. Airborne Topographic Mapper processing parameters.

\begin{tabular}{|l|l|}
\hline \multicolumn{1}{|c|}{ Feature } & \multicolumn{1}{c|}{ Functionality } \\
\hline "con_dir" & $\begin{array}{l}\text { Sets the directory path of the .qi files to be } \\
\text { processed. Separate the .qi files separated by } \\
\text { mission date. }\end{array}$ \\
\hline ymd & $\begin{array}{l}\text { The date the mission was flown in a yyyymmdd } \\
\text { format [for example, 20011008]. }\end{array}$ \\
\hline "dir" & $\begin{array}{l}\text { Sets the output directory organized into the index } \\
\text { tile tiling scheme. }\end{array}$ \\
\hline searchstr="" & $\begin{array}{l}\text { Searches for these files to process [for example, } \\
\text { searchstr="*qi"]. }\end{array}$ \\
\hline name & $\begin{array}{c}\text { Set this function to append to the beginning of the } \\
\text { processed file names. }\end{array}$ \\
\hline
\end{tabular}

command line. This will open a help document explaining the parameters to include in the ATM merging command (table 13). To merge the data, type the following command: merge qi_tiles, "dir", glob="" [press enter]. For example: merge_qi_ tiles, "/training/Index_Tiles/" glob="*.pbd" [press enter].

Table 13. Airborne Topographic Mapper merging parameters.

\begin{tabular}{|l|l|}
\hline \multicolumn{1}{|c|}{ Feature } & \multicolumn{1}{c|}{ Functionality } \\
\hline "dir" & Sets the directory path of the .qi files to be merged. \\
\hline glob="" & $\begin{array}{l}\text { Searches for these files to process [for example, } \\
\text { glob="*..pbd"]. }\end{array}$ \\
\hline srt & $\begin{array}{l}\text { If set to 1, data will be sorted by seconds of the } \\
\text { epoch before being written to the merged file. } \\
\text { This is off by default. }\end{array}$ \\
\hline
\end{tabular}

After processing and merging, the ATM data should be converted into the QQ tiling format. See the ALPS 2-km to Quarter Quadrangle Conversion section for the description of this tiling format.

Filtering the ATM data is a separate function from filtering the EAARL data. Type in the command line: help, rcf_atm_pbds. The help document that opens outlines the possible parameters for this filtering function (table 14). Type in the command line:

rcf_atm_pbds, "ipath", searchstr="', buf=, w=, meta=1 [press enter]. For example: rcf_atm_pbds, “/training/jboniste/Index_ Tiles/", searchstr="*.pbd", buf=2500, w=1200, meta=1 [press enter]. This function uses only the rcf filter because ATM data contain only first return topography.

At this point in the processing, if the user wants to convert the data files from ellipsoid heights to orthometric heights, the user must use the batch_datum_convert function. The help document can be accessed from the CmdLine
Table 14. Airborne Topographic Mapper filtering parameters.

\begin{tabular}{|c|c|}
\hline Feature & Functionality \\
\hline "ipath" & The input directory for the .pbds to be filtered. \\
\hline ifname="" & $\begin{array}{l}\text { Pathname of an individual file that the user wants } \\
\text { to filter. }\end{array}$ \\
\hline searchstr="" & $\begin{array}{l}\text { Searches for the files to process [for example, } \\
\text { searchstr="*n88*.pbd"]. }\end{array}$ \\
\hline buf & $\begin{array}{l}\text { Sets the horizontal size of the area to be filtered in } \\
\text { centimeters. }\end{array}$ \\
\hline W & Sets the vertical height of the area in centimeters. \\
\hline meta & $\begin{array}{l}\text { Set to } 1 \text { to include the filtering parameters in the } \\
\text { file name, otherwise set to } 0 \text {. }\end{array}$ \\
\hline opath="" & $\begin{array}{l}\text { Set equal to the output directory for the files. It will } \\
\text { default to the same directory as the original .pbd } \\
\text { files. }\end{array}$ \\
\hline
\end{tabular}

menu on the Process EAARL Data window. See the Datum Conversion section of this manual for more details regarding this function.

Once the ATM QQ tiles have been filtered, all remaining functions would operate the same as they would with EAARL data. These functions include manual editing and export as American Standard Code for Information Interchange (ASCII) XYZ or American Society for Photogrammetry and Remote Sensing (ASPRS) Log ASCII Standard (LAS). Refer to the Manual Filtering/Editing, Conversion to ASCII XYZ format, and Conversion to ASPRS LAS format sections of this manual that deal with the editing of point data and product creation.

\section{Manual Filtering/Editing}

Several manual editing tools in ALPS remove outliers missed by the batch filtering process and restore valid points. To start the manual editing process, it is usually beneficial to load a boundary area for the data. This is done the same way as loading an image. (See Loading Other Images section of this manual to complete this task.)

If the windows ytk, eaarl, and Process EAARL Data are not currently open, see the Opening ALPS Processing Windows section for instructions. Opening these windows will load a series of files that are necessary for the manual editing of the data to begin.

Manual editing is done by loading each $2-\mathrm{km}$ by $2-\mathrm{km}$ or QQ data tile. Go to the Process EAARL Data window >> File $\gg>$ Read Binary Data File. Navigate to the data tiles directory and select one of the filtered .pbd files [for example, t_e575000_n2768000_17_n88_v_b700_w50_n3_merged_ircf. $p b d]$. The Variable will automatically load. Confirm the Mode is appropriate, whether it is the First Return Topography, Bare Earth Topography, or Submerged Topography. Select 
the Histogram Elevations to display the data properly. (See Viewing Processed Topography Data section.) Confirm that distribution of the data is positioned correctly when viewing the histogram (most coastal areas will center near zero). This ensures the datum was properly applied to the data. A larger graph size will allow a better view of the data. To change the graph size, go to the Graph menu and select 100 Dpi to plot a larger graph size (click Plot).

\section{UTM Zone Fixing}

This function scans through the selected .pbd files to ensure the coordinates in each file are properly zoned. Run this function if the collected data cross UTM zones and before completing the Keep/Remove/Elevation Clipper Filter Tools or Replace/Datum-Convert Filter Tools sections. This function will overwrite the .pbd files with "corrected" versions of the same file. If there is nothing to fix in a file, the function will not change that file. In the case that a mistake is made, a copy of the data should be created before running the function [for example, cp-r dir1 dir2].

To zone fix 2-km data tiles, type in the command line (table 15): help, batch_fix_dt_zones. To run the function, type in the command line in this specific order: batch_fix dt_zones, "dir", glob="" [press enter]. For example: batch_ fix_dt_zones, "/training/Index_Tiles/" glob="*.pbd" [press

Table 15. Fix Universal Transverse Mercator or Quarter Quadrangle zones.

\begin{tabular}{|l|l|}
\hline \multicolumn{1}{|c|}{ Feature } & \multicolumn{1}{c|}{ Functionality } \\
\hline "dir" & The input directory for the .pbds. \\
\hline glob="" & $\begin{array}{l}\text { Searches for the files to fix [for example, } \\
\text { glob="* rcf*.pbd"]. The default search } \\
\text { pattern is "**.pbd" }\end{array}$ \\
\hline
\end{tabular}

enter].

To zone fix QQ data tiles, type in the command line: help, batch_fix_qq_zones. To run the function, type in the command line in this specific order: batch_fix_qq_zones, "dir", glob="" [press enter]. For example: batch_fix_qq_ zones, "/training/lndex_Tiles/" glob="*.pbd" [press enter].

\section{Keep/Remove/Elevation Clipper Filter Tools}

When working with topographic data, water areas should be removed (fig. 24); if editing submerged topography, the deeper sections in the data should be removed. This can be done by using the Keep or Remove filter tools. Remove will delete all of the data within the selected region. Keep will keep all of the data within the selected region.

When working within a bounded area, such as National Park Service (NPS) boundaries, the bounded area should be loaded behind the data during editing. This will ensure proximity to the boundary without removing too much or too little data.

\section{Remove Tool}

To use the Remove tool, go to the Filter tools drop-down menu in the Process EAARL Data window (fig. 26). Confirm that the Input Variable and Data type are correct. In the Remove points using drop-down menu, select Rubberband Box or Points in Polygon. Click Go and click $O K$ on the next window that opens. Begin selecting the region to delete. If the tool is used more than once to remove multiple sections of the data, the plotted data will not update to show these deletions until the user clicks Plot. The user can undo the last section that was removed with Points in Polygon, Rubberband Box, and Single Pixel methods, but this does not apply when using PIP-Thresh removal or Keep (fig. 26) methods.

\section{Keep Tool}

Using the Keep tool is the same as using the Remove tool, but the Keep tool deletes all of the data outside of the selected polygon (fig. 26). This is helpful in island areas where a small amount of data is kept (fig. 27). Go to the Filter tools drop-down menu and select the Keep tool (fig. 26). The Input Variable and Output Variable are labeled the same and match the variable that is being edited. The Data Type should also be the same as the Mode in the Process EAARL Data window. In the Keep points using drop-down menu, select Rubberband Box or Points in Polygon. Click OK on the next window that opens. Begin selecting the region to keep. If the tool is used more than once to remove multiple sections of the data, the plotted data will not update to show these deletions until the user clicks Plot. It is better to use the Remove tool in the case that a mistake is made.

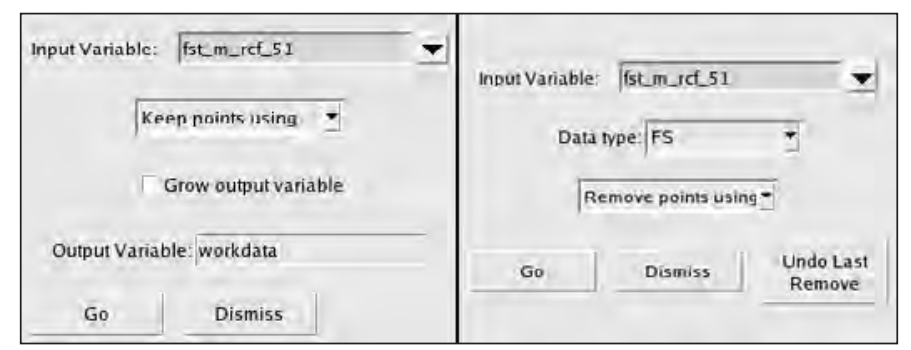

Figure 26. Keep (left) and remove (right) filter windows utilized during manual editing.

\section{PIP-Thresh Removal Tool}

The PIP-Thresh tool is best utilized in removing outliers displayed in certain sections of the histogram. Go to the Remove tool and select PIP-Thresh in the Remove points using drop-down menu. The Max. Threshold is where the maximum 


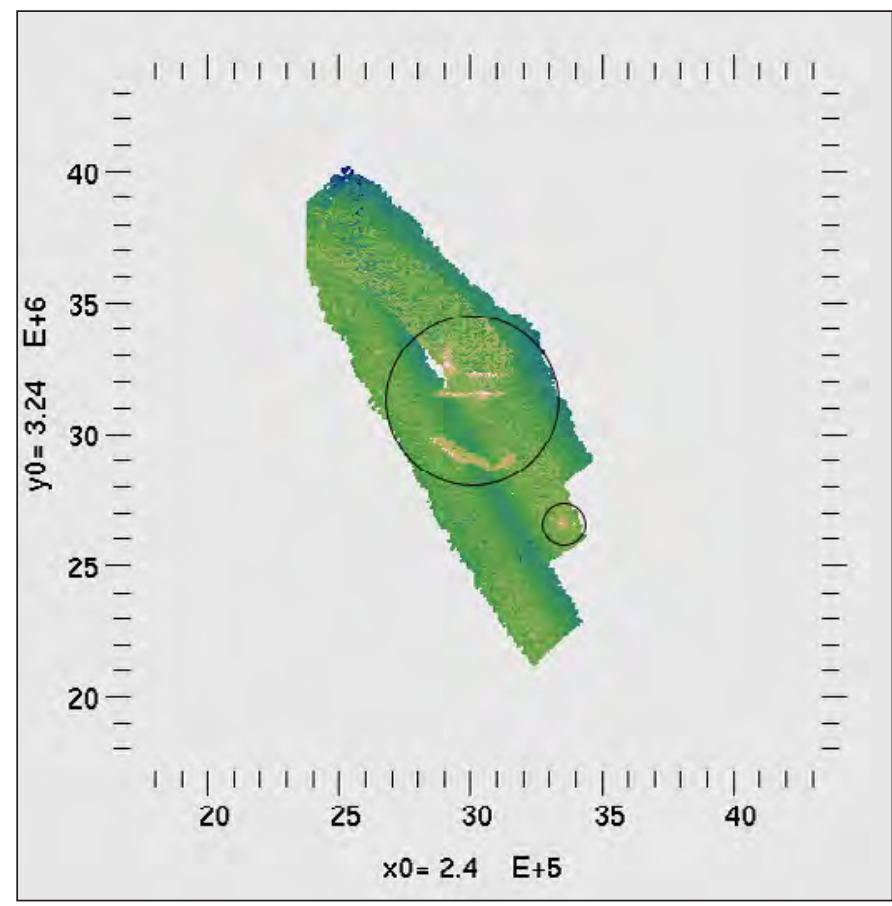

Figure 27. Figure illustrating the use of the keep tool to retain data within the circled areas. Data outside of these areas will be removed.

elevations, Cmax, is selected, and the Min. Threshold is where the minimum elevation, Cmin, is selected. The PIP-Thresh tool will remove only the selected points from the current,

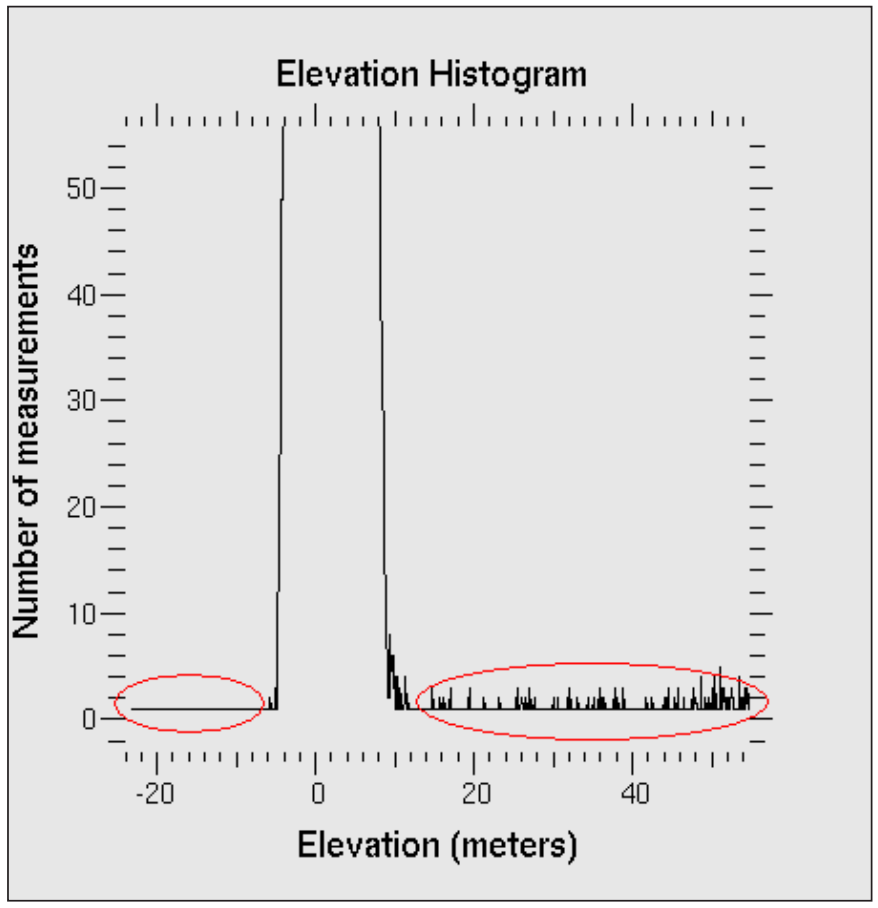

Figure 28. A point-cloud elevation histogram shows a welldefined peak near $0 \mathrm{~m}$. Possible outliers located to the left and right of the peak are circled in red. visible color bar values; for example, if the data are displayed between Cmin: $-5.00 \mathrm{~m}$ and Cmax: $0.50 \mathrm{~m}$, then only the data between these values will be removed by the PIP-Thresh removal method. The user cannot do this with the Keep or other Remove tools, which will remove all data within the selected area, whether displayed on the graph or not.

Possible outliers are represented by the flatter sections on either side of the histogram peak (fig. 28). Select the Histogram Elevations button and start zooming in to zero on the histogram y-axis until the values range from 10 to -10 measurements. This will exaggerate the elevations at which outliers may occur in the data. Try to ascertain whether the outliers are valid or represent true land features. This can be determined by plotting these areas on the graph and looking at imagery. Sources of imagery to consider include the RGB and CIR imagery that accompanies the EAARL data, Google Earth imagery, or other orthophotography.

Select the "flatter" areas on one side of the histogram peak as the Cmin and Cmax values. Click Plot to display the data points on the graph. If the points appear to be an outlier, click Go on the PIP-Thresh tool bar. Then select the data to be omitted via a polygon. Ensure that the Input Variable is the variable that is being edited. Click the Histogram Elevations button again and the specified section of data will be deleted from the rest of the points. Repeat the steps in this section until all of the outliers have been removed from the histogram.

\section{Elevation Clipper Tool}

The Elevation Clipper is another way to remove outliers on either side of the histogram. Instead of selecting specific points to be removed from the data as with PIP-Thresh, this function will "clip" the data on either side of the histogram.

Select the Histogram Elevations button and start zooming in to zero on the histogram y-axis until the values range from 10 to -10 measurements. Select $\mathrm{Cmin}$ and $\mathrm{Cmax}$ to be displayed on the graph. Click Plot to view the data. If the data are outside of the selected histogram limits, they are to be deleted. Click the Elevation Clipper button (fig. 29). When the window opens, ensure that the Input Variable and the Output

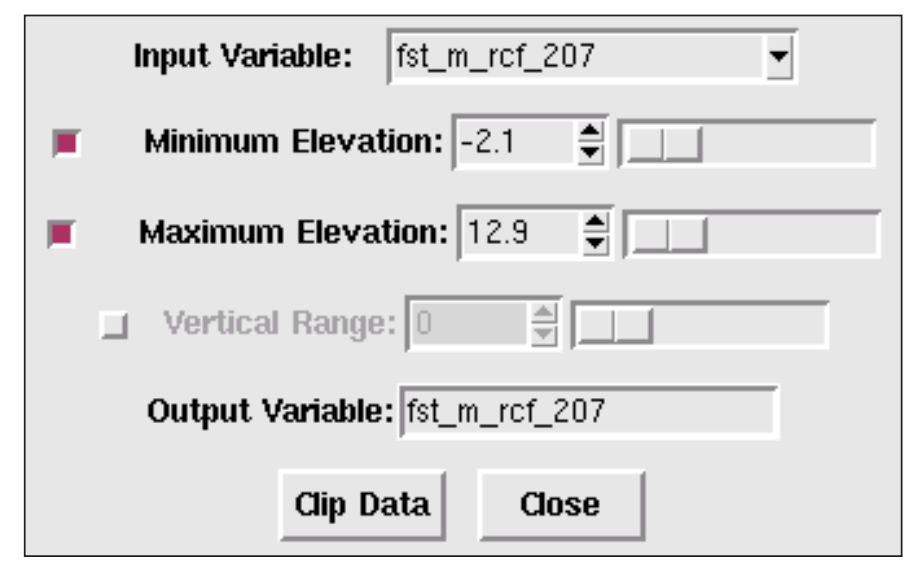

Figure 29. The elevation clipper window. 
Variable are the same and match the variable that is being edited. Also review the Minimum Elevation and Maximum Elevation to see that they are the same as the Cmin and Cmax values on the Process EAARL Data window (click Clip Data). Click the Histogram Elevations button again. Confirm that the data outside of the selected histogram were removed.

\section{Saving Manual Edits}

To save edits, select Write Binary Data File from the File menu on the Process EAARL Data window. Select the Output Path to be the same as the input path. Add_mf to the end of the file name that was edited, then click Save. This will save the edited file within the specified directory.

\section{Replace/Datum-Convert Filter Tools}

The Replace function is used to replace data that were not intended for removal during the filtering process. This often occurs in bare earth data in areas where building density is high and valid, elevated topographic features exist. The filter that was used to filter the last return data to produce a bare earth surface removes most of the buildings. However, valid topographic features surrounding the buildings can be inadvertently removed also.

To replace valid data to the filtered data, load the filtered data. Go to the Options menu in the Process EAARL Data window and select Constant Colorbar for Variables. This will ensure that other loaded variables will have the same color bar values as the data that are being edited. Load in the merge-data file that was created before the filter was applied to the data. This merged file was created from the write_merge parameter used during the batch filter process. Go to the File menu in the Process EAARL Data window, select Read Binary Data file, and select the *merged.pbd file. The file will load into the Variable menu as merged_v.

If the data being edited have been converted to NAVD88 elevations, then the merged variable must also be converted to NAVD88. Go to the PostProcessing menu or the Filter tools menu and select Datum-Convert. The Input Variable should be merged_v; if it is not, select it from the drop-down menu and click Go. The command terminal may ask for a UTM zone number; enter the zone number at this time. Watch for the return on the command terminal to indicate the datum conversion has finished. Select n88_merged_v from the Variable list. Unselect the Auto Fma button and click Plot to have the unfiltered data plotted over the filtered data.

Click the Filter tools drop-down menu and select the Replace function (fig. 30). The Original Data Variable will be the datum converted merged data [n88_merged_v] and the Input Variable will be the filtered data that needs to be replaced [bet_m_ircf_m_g]. Click on the Same as input variable button to have the Output Variable be the same as the input. The Output Variable can be named something else such as finaldata. If it is named finaldata, this becomes the variable name for the data. In the Select points to replace using drop-

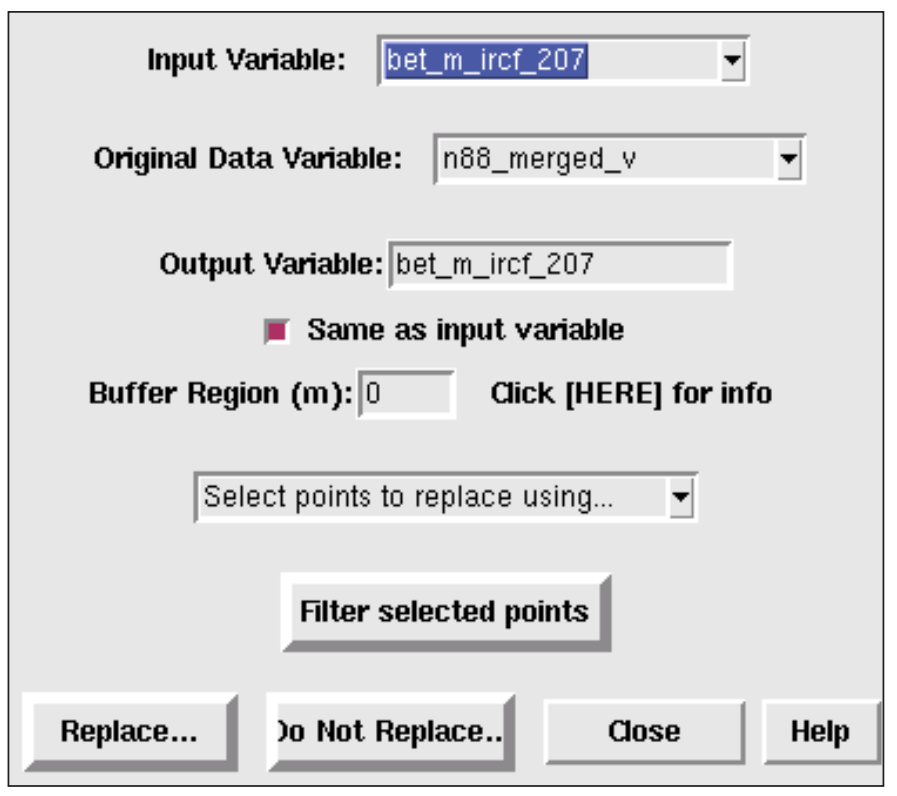

Figure 30. The replace function window.

down menu, select Points in Polygon, Rubberband Box, or Window Limits. If prompted, click $O K$ on the next window that opens. Select the area of points to be replaced; no selection is required with Window Limits function. Click Filter selected points and the RCF window appears. Use either the RCF or IRCF filter to edit the area manually. See the Filtering section of this manual for further instructions on using the filter. The filter will use the Input Variable workdata and the Output Variable rcf_workdata. Click Go. The variable rcf_workdata will load into the variable list. It is recommended to plot $r c f_{-}$ workdata on a graph to determine whether the replacement is an improvement.

If the results are satisfactory, click Replace. Click Yes in the next window to add the extracted data to the filtered variable. If the results are not satisfactory, click Do Not Replace and click Yes. Repeat the above steps until all of the removed areas have been replaced.

See the Saving Manual Edits section of this manual to save the edited file.

\section{Quality Control}

Accuracy of the produced data depends on the configuration of the lidar system, GPS satellite arrangement, and the type of surface being mapped. Research into the accuracy of the EAARL system has shown that elevation root mean square errors (RMSE) range from 10 to $14 \mathrm{~cm}$ for submerged topography to 16 to $20 \mathrm{~cm}$ for sub-canopy topography (Nayegandhi and others, 2009). To ensure optimum data accuracy, quality control (QC) procedures have been established. These procedures examine the removal of erroneous points, data anomalies, or any potential horizontal or vertical bias. Anomalies may result from the misalignment 
of an axis from roll, pitch, or yaw, system timing offsets, atmospheric conditions, GPS bias, or spectral conditions of terrain.

Steps used to the ensure accuracy of the delivered product include: (1) examination of the survey area for errors along the edge of the swath (fig. 31); (2) verification of GPS flight trajectories (fig. 32); (3) use of ground-control data collected along flat/uniformly sloping terrain, not in areas where vegetation is too dense for lidar penetration; (4) use of RMSE calculations between multiple surveys of the same area to estimate both horizontal and vertical accuracy (vertical accuracy of any DEM is defined as 1.96 times RMSE linearly interpolated elevations in the DEM).

\section{First Return Quality Control Specifics}

Remove the flat extension on either side of the histogram, if not performed during the manual editing. If reviewing a coastal area, the coastline should be seamless and smooth. Also remove any erroneous data collected during the turn of the aircraft, data that may have been left during the manual editing process. Look at the elevation values to see that the datum conversion worked properly. The vegetation and infrastructure should be included in the first return data; review the data to confirm these are included. If multiple collection days exist, complete the mtransect function on a few data tiles. This function will verify the relation between survey days. If there is a difference, it is most likely a GPS

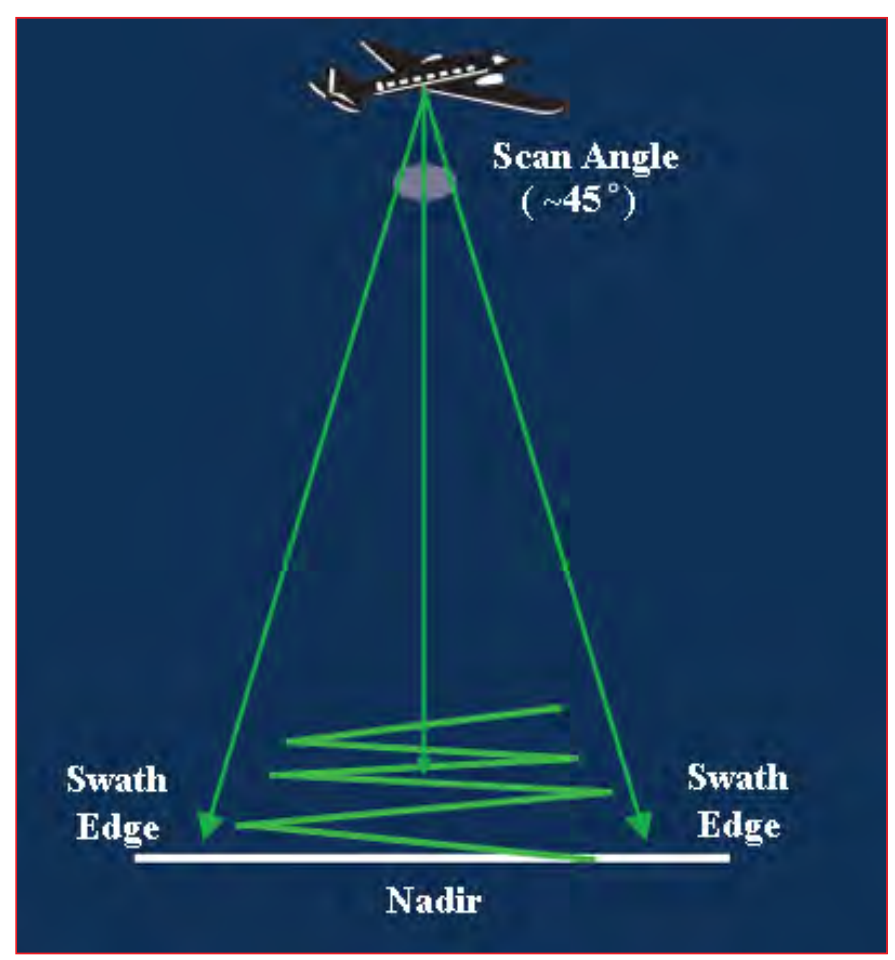

Figure 31. A considerable amount of data can be created along the edge of the swath (Nayegandhi and others, 2006). error that exists between the collection days. Review the Determining the Roll Bias section of the manual for directions on the mtransect function. After the preceding steps have been completed, add $q c$ to the end of the filename [t_e_240000 n3338000_16_n88_v_b500_w600_n3_merged_fs_rcf_mf qc.pbd].

\section{Bare Earth Quality Control Specifics}

Remove the flat extension on either side of the histogram, if not performed during the manual editing. If reviewing a coastal area, the coastline should be seamless and smooth. Remove any erroneous data collected during the turn of the aircraft, data that may have been left during the manual editing process. Review the elevation values to ensure that the datum conversion worked properly. All infrastructure and vegetation should be removed from bare earth data. The filter should remove most of these data, but there could be areas that still need removal after the filter or manual editing. If multiple collection days exist, complete the mtransect function on a few data tiles. Review the Determining the Roll Bias section of the manual for directions on the mtransect function. If ground-control data exist, find a method of analysis to test data integrity between ground-control data and lidar collection. Compare survey years from the same area. Often data collection occurs over multiple years in the same area to evaluate change. Take an area that is known to be stable over time, such as a parking lot or airport tarmac, then

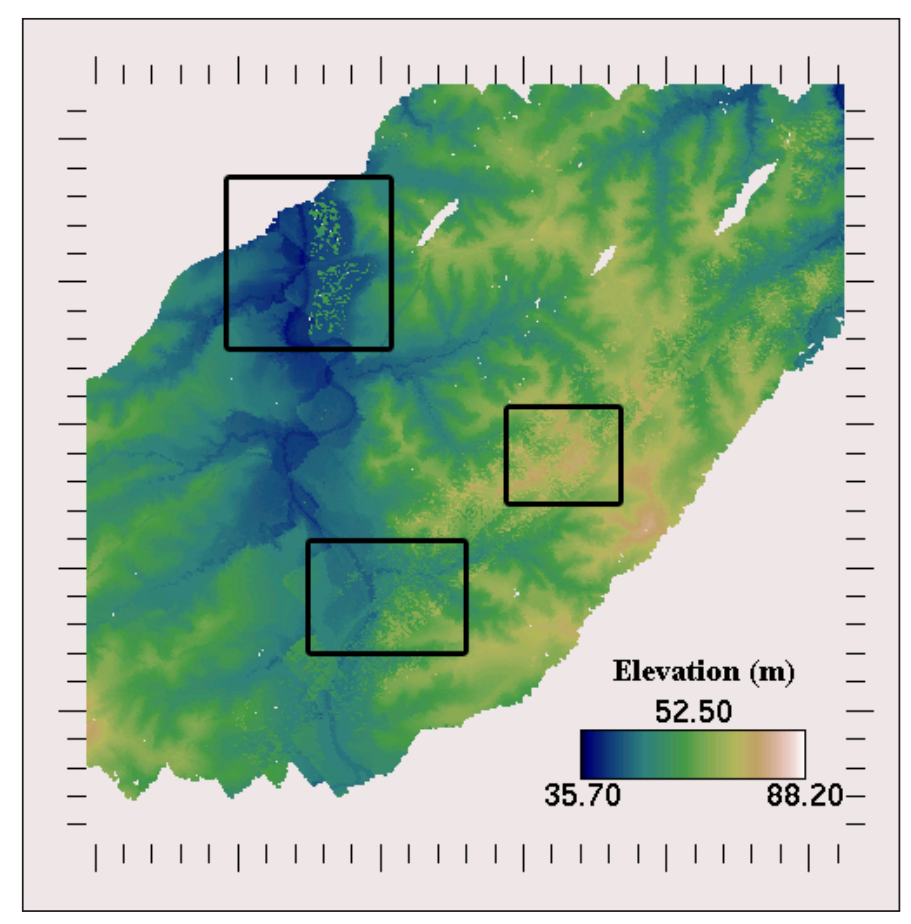

Figure 32. An error in the data can be seen within each square of this bare earth 2-km by 2-km tile. The GPS offset occurred between the 2 days of data collection. 
compare these areas against one another. After the preceding steps have been completed, add $q c$ to the end of the filename [t_e_240000_n3338000_16_n88_v_b500_w60_n3_merged_rcf mf_qc.pbd].

\section{Product Creation}

The final step is to convert the data to point cloud data (ASCII .xyz or ASPRS .las) or grid the data into DEM products. The DEM is usually created by using Delaunay Triangulation, followed by interpolation to create a triangular irregular network (TIN). The output data type is usually a geotiff (32-bit floating-point format) that includes georeferencing information.

Table 16. ASCII XYZ conversion parameters.

\begin{tabular}{|c|c|}
\hline Feature & Functionality \\
\hline "dirname" & $\begin{array}{l}\text { Sets the directory name where the input files } \\
\text { reside [for example, "'/training/katrina/ } \\
\text { Index_Tiles/"]. }\end{array}$ \\
\hline outdir="" & $\begin{array}{l}\text { Set to the output directory name to write files } \\
\text { to this location. The default will be the same } \\
\text { path as the input directory. Set up a new } \\
\text { directory for the ASCII .xyz files [for example, } \\
\text { outdir="/training/katrina/be_xyz/"]. }\end{array}$ \\
\hline mode & $\begin{array}{l}\text { Set to } 1 \text { for the first return, } 2 \text { for bathymetry, and } \\
3 \text { for bare earth. This will also append fs, ba, } \\
\text { or be into the output file name. }\end{array}$ \\
\hline zone & Set to UTM zone number. \\
\hline$S S=" "$ & $\begin{array}{l}\text { Searches for data used in the find command. } \\
\text { This will override other search options, such } \\
\text { as rcfmode, datum, readpbd, and readedf [for } \\
\text { example, ss="*'rcf*'mf*.pbd"]. }\end{array}$ \\
\hline rcfmode & $\begin{array}{l}\text { Set to } 1 \text { to convert RCF'd files, set to } 2 \text { to convert } \\
\text { IRCF'd files, and set to } 3 \text { to convert IRCF_mf } \\
\text { files. This option is considered an alternative } \\
\text { to the search string. }\end{array}$ \\
\hline buffer & $\begin{array}{l}\text { Sets buffer to size in meters. Data outside } \\
\text { the tile's limits plus the buffer's size will be } \\
\text { excluded. A negative value indicates that all } \\
\text { data should be used. }\end{array}$ \\
\hline atm & Set to 1 if converting ATM data. \\
\hline qq & $\begin{array}{l}\text { Set to } 1 \text { to convert the quarter quadrangle tiles } \\
\text { into the ASCII format. }\end{array}$ \\
\hline update & $\begin{array}{l}\text { Set to } 1 \text { to convert only those files that have not } \\
\text { been converted to the ASCII .xyz format. This } \\
\text { will not affect already converted files. }\end{array}$ \\
\hline
\end{tabular}

\section{Conversion to ASCII XYZ Format}

To begin the conversion to ASCII.$x y z$, open the following windows: $y t k$, eaarl, and Process EAARL Data. (See the Opening ALPS Processing Windows section for instruction.) Set the curzone/utmzone [for example, curzone $\mathbf{1 6}$ or utmzone=16]. Type in the command line: help, batch_write_xyz. Alternatively, go to batch_write_xyz within the CmdLine menu in the Process EAARL Data window. This will open a help document explaining which parameters should be included in the conversion to ASCII .xyz (table 16).

Once the appropriate parameters have been determined, type the following command: batch_write_xyz, "dirname", outdir="', mode=, zone=, ss="', buffer= [press enter]. For example: batch_write_xyz, "/training/Index_Tiles/", outdir= "/training/fs_xyz/", mode=1, zone=16, ss="*fs"_qc.pbd", buffer $=10$ [press enter].

Other options can be used when converting .pbd data to ASCII .xyz; review the help document for those options. Upon function completion, a return will appear in the command prompt.

\section{Conversion to ASPRS LAS Format}

In order to begin the ASPRS .las conversion, open the following windows: $y t k$, eaarl, and Process EAARL Data. (See the Opening ALPS Processing Windows section for instruction. Set the curzone/utmzone [for example, curzone=16 or utmzone=16]. Type in the command line help, batch_pbd2las. Alternatively, go to batch_pbd2las within the CmdLine menu in the Process EAARL Data window. This will

Table 17. ASPRS LAS conversion parameters.

\begin{tabular}{|c|c|}
\hline Feature & Functionality \\
\hline "con_dir" & $\begin{array}{l}\text { The directory name where the input files reside } \\
\text { [for example, "/training/katrina/Index_- } \\
\text { Tiles/"]. }\end{array}$ \\
\hline searchstr="" & $\begin{array}{l}\text { Searches for data used in the find command [for } \\
\text { example, searchstr="**rcf*mf*.pbd"]. This } \\
\text { will override other search options such as } \\
\text { nad83, wgs84N, and wgs84S. }\end{array}$ \\
\hline zone_nbr & Sets the UTM zone number \\
\hline typ & $\begin{array}{l}\text { Set to } 1 \text { if converting first return, } 2 \text { for bare } \\
\text { earth, and } 3 \text { for submerged topography. }\end{array}$ \\
\hline buffer & $\begin{array}{l}\text { Specifies buffer in meters used to apply to tile } \\
\text { boundary. If set to -1 (default), all data will be } \\
\text { used. }\end{array}$ \\
\hline qq & $\begin{array}{l}\text { Set to } 1 \text { to convert quarter quadrangle tiles into } \\
\text { ASPRS .las format. }\end{array}$ \\
\hline update & $\begin{array}{l}\text { Set to } 1 \text { to process only those files that have not } \\
\text { been converted to a ASPRS .las format. This } \\
\text { will not affect already converted files. }\end{array}$ \\
\hline
\end{tabular}


open a help document explaining which parameters should be included in the conversion to ASPRS .las (table 17).

Once the appropriate parameters have been determined, type the following command:

batch_pbd2las, "con_dir", searchstr="', zone_nbr=, typ=, buffer= [press enter]. For example: batch_pbd2las, “/training/ Index_Tiles/", searchstr="*fs*_qc.pbd", zone_nbr=16, typ=1, buffer=10 [press enter].

Other options can be used when converting to ASPRS .las; review the help document for those options. Upon function completion, a return $[>]$ will appear in the command prompt.

A series of scripts has been created for the interactive data language (IDL) to read EAARL data format (.edf) files created in ALPS, triangulate them based on area and distance thresholds, and create a grid based on the triangulation.

In order to begin the .pbd to .edf batch conversion, open the following windows: ytk, eaarl, and Process EAARL Data. (See the Opening ALPS Processing Windows section for instruction.) Begin in ALPS by converting the manually edited .pbd into .edf. Type in the command line: \#include "batch_ typ_convert.i", then type help, batch_pbd2edf to open the help document listing possible parameters (table 18). Type the following command: batch_pbd2edf, "dirname", searchstr="" [press enter]. For example: batch_pbd2edf, "/training/lndex_ Tiles/", searchstr="*_qc.pbd" [press enter].

Table 18. pbd2edf conversion parameters.

\begin{tabular}{|l|l|}
\hline \multicolumn{1}{|c|}{ Feature } & \multicolumn{1}{c|}{ Functionality } \\
\hline "dirname" & $\begin{array}{l}\text { The directory name where the input files reside } \\
\text { [for example, "/training/katrina/Index_ } \\
\text { Tiles/"]. }\end{array}$ \\
\hline searchstr=" & $\begin{array}{l}\text { Searches for data in the find command [for } \\
\text { example, searchstr="*rcf*mf*.pbd"]. This } \\
\text { will override other search options, such as } \\
\text { rcfmode, onlymf, n88, and w84. }\end{array}$ \\
\hline
\end{tabular}

Upon function completion, a return [ $>$ ] will appear in the command terminal. The .edf files are ready for use in IDL (as long as IDL is installed). Open another shell or terminal in Linux. The user must migrate to the following directory to begin processing: cd /opt/eaarl/lidar-processing/idl [press enter]. Once in the directory, type idlde; the user will then see IDL load. Go to the File menu and click Open. A window will open with scripts; select the following: batch_grid.pro and grid_eaarl_data.pro [click OK]. Go to the Run menu and select Compile All. The processing window will state Compiled module: BATCH_GRID, BATCH_MAKE_GE_PLOTS, GRID_EAARL_DATA,PLOT_EAARL_GRIDS, PLOT_ZBUF_ EAARL_GRIDS, MAKE_GE_PLOTS, and WRITE_GEOTIFF. The parameters will display in the top main window (table 19). Type the following command in the $I D L>$ command line: batch_grid, "directory", write_geotiffs=1, utmzone=, mode=,
Table 19. Interactive Data Language DEM creation parameters.

\begin{tabular}{|c|c|}
\hline Feature & Functionality \\
\hline "filename" & $\begin{array}{l}\text { The directory name where the input files reside } \\
\text { [for example, "/training/katrina//ndex_ } \\
\text { Tiles/"]. }\end{array}$ \\
\hline cell & Sets the grid cell dimension. The default is $1 \mathrm{~m}$. \\
\hline mode & $\begin{array}{l}\text { Set to } 1 \text { for the first surface, } 2 \text { for bathymetry, } \\
\text { and } 3 \text { for bare earth. }\end{array}$ \\
\hline searchstr="" & $\begin{array}{l}\text { Searches for data used in the find command [for } \\
\text { example, searchstr="*'rcf*'mf*.edf"]. }\end{array}$ \\
\hline write_geotiffs & Set to 1 to write out geotiffs. \\
\hline utmzone & Sets UTM zone number. \\
\hline area_threshold & $\begin{array}{l}\text { This is the maximum allowable area of a } \\
\text { triangulated facet. The default for this value } \\
\text { is } 200 \text { square meters. }\end{array}$ \\
\hline dist_threshold & $\begin{array}{l}\text { This is the maximum allowable distance } \\
\text { between two vertices of the triangulated } \\
\text { facet. The default for this value is } 50 \text { meters. } \\
\text { Increase this value to reduce "holes" in the } \\
\text { data. }\end{array}$ \\
\hline
\end{tabular}

searchstr="” [press enter]. For example: batch_grid, "/ training/Index_Tiles/", write_geotiffs=1, utmzone=16, mode=1, searchstr $="$ *edf" [press enter].

Other options can be used when gridding; review the script for those options. The processing window will inform the user of gridding completion.

\section{GlobalMapper DEM Creation}

\section{ALPS 2-km Data Tile DEM Creation}

A script has been created for the geographic information system (GIS) software GlobalMapper ${ }^{\mathrm{TM}}$ to read the threedimensional point data (ASCII .xyz), triangulate it, and create a grid based on the triangulation. The script eliminates points from the grid by comparing the distance from each point to a real data point. The threshold value establishes the distance to a real data point. This value controls the gaps within and along the edges of the dataset. The edges are problematic areas. Large triangles can be created to cover a large spatial area in which no real data points exist. The threshold will tell the script how close to trim those areas. If the threshold is made too small, then areas of points become isolated rather than becoming a smooth product. If the threshold is made too large, then a "collar" begins to form around the data.

To be able to use the $g m \_x y z 2 d e m$ script, Active TCL (Version 8.4.17.0) and GlobalMapper must be installed. Double click on the $g m \_x y z 2 d e m$ script and a window will 


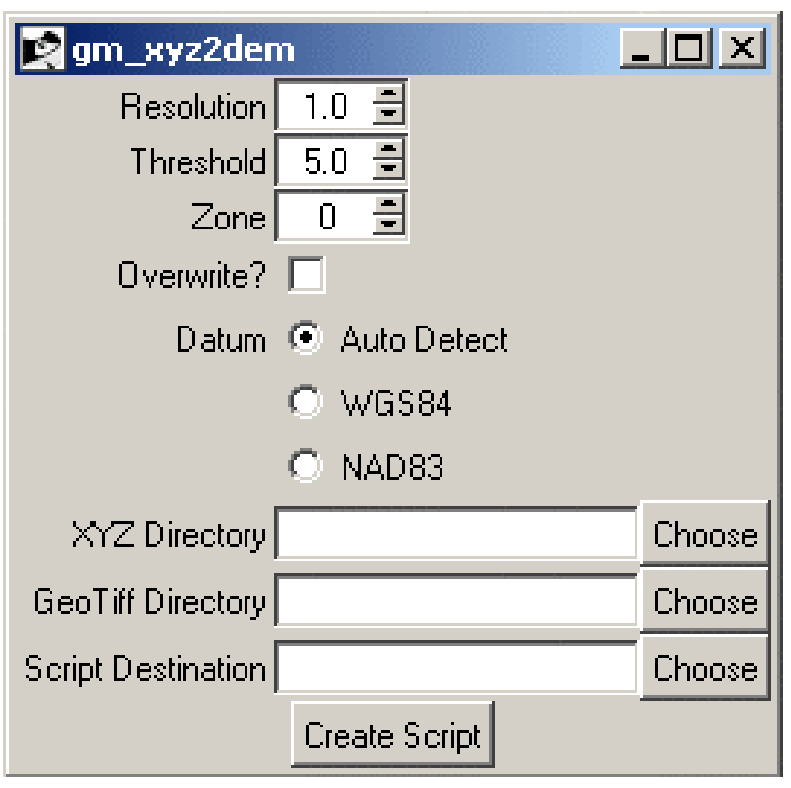

Figure 33. Window that shows DEM script created for GlobalMapper.

open (fig. 33). Keep the following values the same: Resolution at 1 , Threshold at 5; the zone and datum will automatically be detected from the file names. This script will only work for data tiles in the 2-km by 2-km data tile format. See the Quarter Quadrangle Data Tile DEM Creation section of this manual to convert QQ data into DEMs. Click the Choose button to select an input directory of ASCII .xyz data for the XYZ Directory and then to select an output directory for the GeoTiff Directory. For the Script Destination, name the script and click on the Choose button to select the location of the script. Remember the script location because it will be used again. Add .gms to the end of the script name [for example, runme.gms]. Once the script is saved, click the Create Script button. A window will appear with Your script has been created. Open GlobalMapper and go to the File menu and click Run Script. Navigate to the directory where the saved .gms script is located and select it. The script processing window opens. Click Run Script button. This process will take approximately 15 minutes to run. When it has finished, click close.

Open the newly created DEM files in GlobalMapper by clicking Open your own data files or go to the File menu and click Open Data File(s). Navigate to the designated output directory and the files are displayed.

\section{Quarter Quadrangle Data Tile DEM Creation}

To create DEMs from QQ-formatted data, convert the EAARL or ATM data into 2-km data tiles. (See the section on Quarter Quadrangle to ALPS 2-km Conversion.) To create geotiffs, complete the steps in Interactive Data Language DEM Creation. Copy the geotiffs into a separate directory and then onto a local machine. Review all geotiffs and delete any that are empty. Solid red or black squares represent empty
Table 20. Quarter Quadrangle geotiff preparation parameters.

\begin{tabular}{|l|l|}
\hline \multicolumn{1}{|c|}{ Feature } & \multicolumn{1}{c|}{ Functionality } \\
\hline "tif_dir" & $\begin{array}{l}\text { Designates the location of the 2-km geotiff } \\
\text { directory } \\
\text { [for example, "/training/katrina/be__ } \\
\text { geotiff/"]. }\end{array}$ \\
\hline "pbd_dir" & $\begin{array}{l}\text { Designates the location of the 00 .pbd directory } \\
\text { [for example, "/training/katrina/be_00_ } \\
\text { tiles/"]. }\end{array}$ \\
\hline mode & $\begin{array}{l}\text { Set as 1 for the first surface, 2 for bathymetry, } \\
\text { and 3 for bare earth. }\end{array}$ \\
\hline outfile & $\begin{array}{l}\text { Generates the output file [for example, " / / } \\
\text { katrina05be.tcl"]. This file is used in the } \\
\text { geotiff creation. }\end{array}$ \\
\hline tif_glob="" & $\begin{array}{l}\text { Searches for specific .tif files from data directory. } \\
\text { Default is *..tif. }\end{array}$ \\
\hline pbd_glob="" & $\begin{array}{l}\text { Searches for specific .pbd files from data quarter } \\
\text { quadrangle directory. Default is *.pbd. }\end{array}$ \\
\hline
\end{tabular}

geotiffs. Sync changes back to the original location. Manually delete the files at the original location or use rsync --delete. Ensure that there are quarter quadrangle .pbd files. Type help, qqtiff_gms_prep to open the help document (table 20). Type the following command: qqtiff_gms_prep, "tif_dir", "pbd_dir", mode, "outfile" [press enter]. For example: qqtiff_gms_prep, "/training//2k_fs_geotiffs/", " "/training/fs_00", 1, "/training/ katrina.tcl" [press enter].

Transfer the output file to a local machine. Copy the gm_tiff2ktoqq.tc/ script from the /opt/eaarl/lidar-processing/ linux- $x p$ directory to a local machine. To be able to use the gm_tiff2ktoqq.tc/ script, Active TCL (Version 8.4.17.0) and GlobalMapper must be installed. Double click on the gm_tiff2ktoqq.tcl script and a window will open (fig. 34). Click Choose in the $2 k$ Tiff Source Path, navigate to the geotiff source directory. Click OK. Click Choose in the 00 Tiff Destination Path, navigate to a created QQ directory. Click $O K$. The Output Naming Scheme specifies a prefix and a suffix to use for the output geotiff filenames. The light gray 12345a6b is a placeholder to show where the quarter quadrangle names

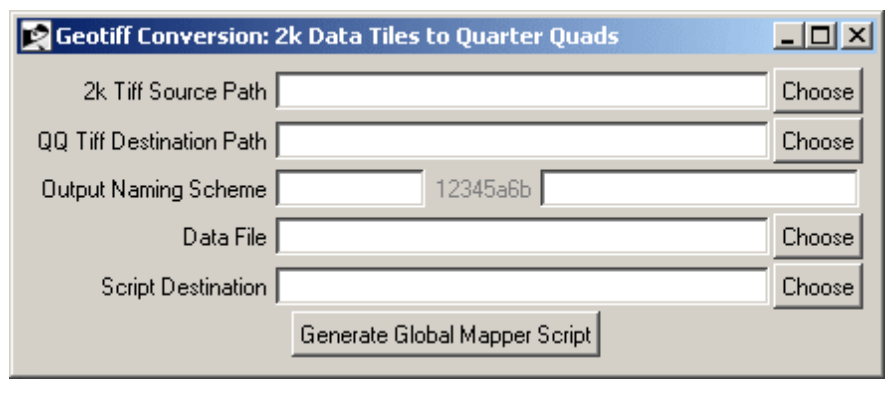

Figure 34. Window that shows GlobalMapper geotiff conversion script. 
will go. Fields may be left blank. Click Choose in the Data File, navigate to the created qqtiff_gms_prep script. Click $O K$. Click Choose in the Script Destination, navigate to the output directory for the GlobalMapper script, and give the script a filename. Click OK. Click Generate Global Mapper Script. A window will open with Your script has been created. The dialog will not close, click the $X$ in the top right corner of the dialog to exit.

Open GlobalMapper and go to the File menu and click Run Script. Navigate to the directory where the saved .gms script is located and select it. The script processing window opens. Click Run Script button. This process will take approximately 15 minutes to run. When it has finished, click close. Open the newly created DEM files in GlobalMapper by clicking Open your own data files or go to the File menu and click Open Data File(s). Navigate to the designated output directory and the files are displayed.

\section{References Cited}

Brock, J.C., Wright, C.W., Clayton, T.D., Nayegandhi, Amar, 2004, LIDAR optical rugosity of coral reefs in Biscayne National Park, Florida: Coral Reefs, v. 23, p. 48-59.

Fischler, M.A., and Bolles, R.C., 1981, Random sample consensus: A paradigm for model fitting with applications to image analysis and automated cartography: Communications of the Association for Computing Machinery, v. 24, p. 381-395.

Harris, M., Brock, J.C., Nayegandhi, Amar, and Duffy, M., 2005, Extracting shorelines from NASA airborne topographic lidar-derived digital elevation models: U.S. Geological Survey Open-File Report 2005-1427, 32 p.

Nayegandhi, Amar, Brock, J.C., and Wright C.W., 2009, Small-footprint, waveform-resolving lidar estimation of submerged and sub-canopy topography in coastal environments: International Journal of Remote Sensing, v. 30 , no. 4 , p. $861-878$.

Nayegandhi, Amar, Brock, J.C., Wright C.W., Clayton, T.D., and Mosher, L.A., 2004, Processing and Filtering 'Bare Earth' Topographic Data Acquired by NASA's Experimental Advanced Airborne Research Lidar (EAARL), in Proceedings of the American Society for Photogrammetry and Remote Sensing (ASPRS) Annual Conference, Denver, CO, 23-28 May 2004, one CD-ROM.

Nayegandhi, Amar, Brock, J.C., Wright, C.W., and O'Connell, M.J., 2006, Evaluating a small footprint, waveformresolving lidar over coastal vegetation communities: Photogrammetric Engineering \& Remote Sensing, v. 72, no. 12, p. 1407-1417.
Sallenger, A.H., and Brock, J.C., 2001, Coastal change hazards during extreme storms investigated with airborne topographic lidar: U.S. Geological Survey Open-File Report 01-98, 2 p.

Shrestha, R., Carter, W., Slatton, C., and Dietrich, W., 2007, "Research-quality" airborne laser swath mapping: The defining factors: LEN - LiDAR Remote Sensing Education Network, Geosensing Engineering and Mapping (GEM) Civil and Coastal Engineering Department University of Florida, accessed on: 1 January 2008. URL: http://www. aspl.ece.ufl.edu/reports/NCALM_WhitePaper_v1.1.pdf.

Wagner, W., Ullrich, A., Melzer T., Briese, C., and Kraus, K., 2004, From single-pulse to full-waveform airborne laser scanners: Potential and practical challenges: International Archives of Photogrammetry and Remote Sensing, v. 35, Part B3, p. 201-206.

Wagner, W., Roncat, A., Melzer, T., and Ullrich, A., 2007, Waveform analysis techniques in airborne laser scanning: International Archives of Photogrammetry and Remote Sensing, v. 36, Part 3, p. 413-418.

Wright, C.W., and Brock, J.C., 2002, EAARL: A lidar for mapping shallow coral reefs and other coastal environments, in Proceedings of the Seventh International Conference on Remote Sensing for Marine and Coastal Environments, Miami, FL, 20-22 May 2002, Veridian International Conferences, one CD-ROM. 


\section{Appendix A. ALPS Installation}

This section documents the steps needed to install ALPS and its required software on a computer running Linux. These instructions require root or superuser access to the machine.

1. Set up directories.

a. Create the following directories for the ALPS system:

sudo mkdir -p /opt/eaarl

sudo chown `id -un `’id -gn`/opt/eaarl

mkdir -p /opt/eaarl/packages/src

mkdir /opt/eaarl/bin

b. Create the following directory and download into the directory all of the files required for the rest of the document.

mkdir /opt/eaarl/tarfiles

2. ActiveTcl

a. Download ActiveTcl 8.4.17.0 from the website. Select the -x86_64.tar.gz file for a 64-bit system. Otherwise, select the -ix86.tar.gz file (most installers will use this). http://downloads. activestate.com/ActiveTcl/Linux/8.4.17/

b. Untar the tarball as a directory in /opt/eaarl/ packages/src/. cd /opt/eaarl/packages/src tar zxvf /opt/eaarl/tarfiles/ActiveTcl8.4.17linux-ix86.tar.gz

c. Run the install script for ActiveTcl. When prompted for an installation location, choose /opt/ eaarl/packages/ActiveTcl8.4.17.

cd ActiveTcl8.4.17-linux-ix86

./install.sh

d. Add these links to ActiveTcl in the /opt/eaarl/bin/ directory: cd /opt/eaarl/bin In -sf /opt/eaarl/packages/ActiveTcl8.4.17/bin/ page

In -sf /opt/eaarl/packages/ActiveTcl8.4.17/bin/ tclsh

In -sf /opt/eaarl/packages/ActiveTcl8.4.17/bin/ tclsh8.4

In -sf /opt/eaarl/packages/ActiveTcl8.4.17/bin/ teacup In -sf /opt/eaarl/packages/ActiveTcl8.4.17/bin/ tkcon In -sf /opt/eaarl/packages/ActiveTcl8.4.17/bin/ wish

In -sf /opt/eaarl/packages/ActiveTcl8.4.17/bin/ wish8.4

3. rlwrap

a. Download rlwrap from the lidar.net server.lidar. net:/mnt/alps/eaarl/tarfiles/rlwrap-0.21.tar.gz b. Untar the tarball as a directory in /opt/eaarl/ packages/src. cd /opt/eaarl/packages/src tar zxvf /opt/eaarl/tarfiles/rlwrap-0.21.tar.gz

c. Compile and install. cd rlwrap-0.21 ./configure --prefix=/opt/eaarl make make install

4. Yorick

a. Download Yorick 2.1.04 from lidar.net. lidar.net:/mnt/alps/eaarl/tarfiles/yorick-2.1.04.tgz

b. Untar the tarball as a directory in /opt/eaarl/ packages/src/. cd /opt/eaarl/packages/src $\operatorname{tar} \mathbf{z x v f} /$ opt/eaarl/tarfiles/yorick-2.1.04.tgz

c. Build yorick. cd yorick-2.1.04 make relocatable

d. Install yorick. cd /opt/eaarl/packages tar zxvf /opt/eaarl/packages/src/yorick-2.1.04/ yorick-2.1-04.tgz

e. Add links to yorick in /opt/eaarl/bin. cd /opt/eaarl/bin In -sf /usr/local/yorick-2.1-04/bin/yorick ln -sf /usr/local/yorick-2.1-04/bin/gist

5. Yorick-Z

a. Download Yorick-Z 1.2 from lidar.net. lidar.net:/mnt/alps/eaarl/tarfiles/yorick-z-1.2.tgz

b. Untar the tarball as a directory in /opt/eaarl/ packages/src/. cd /opt/eaarl/packages/src tar zxvf /opt/eaarl/tarfiles/yorick-z-1.2.tgz

c. Compile and install.

cd yorick-z-1.2

./configure

make

make check

make install

6. Yeti

a. Download Yeti 6.2.1 from lidar.net. lidar.net:/mnt/alps/eaarl/tarfiles/yeti-6.2.1.tar.gz

b. Untar the tarball as a directory in /opt/eaarl/ packages/src. cd /opt/eaarl/packages/src tar zxvf /opt/eaarl/tarfiles/yeti-6.2.1.tar.gz

c. Compile and install.

cd yeti-6.2.1

yorick -batch ./config.i

make

make install 
7. YTK

a. Download YTK 1.0a from lidar.net.

lidar.net:/mnt/alps/eaarl/tarfiles/ytk-1.0a.tgz

b. Untar the tarball as a directory in /opt/eaarl/

packages/src.

cd /opt/eaarl/packages/src

$\operatorname{tar} \mathbf{z x v f} /$ opt/eaarl/tarfiles/ytk-1.0a.tgz

c. Copy the required files to their appropriate places.

cd ytk-1.0a

mkdir/opt/eaarl/packages/yorick-2.1.04/

contrib

cp ytk.i /opt/eaarl/packages/yorick-2.1.04/

contrib/

cp ytk.gif/opt/eaarl/packages/yorick-2.1.04/

contrib/

8. ALPS

a. Obtain ALPS, either through Concurrent Version

Systems (CVS) or as a tarball, and install as /opt/ eaarl/lidar-processing. For the tarball:

cd /opt/eaarl

tar zxvf /opt/eaarl/tarfiles/alps.tar.gz

b. Build and install the ALPS yorick plugins.

cd /opt/eaarl/lidar-processing/yorick-extend/rcf yorick -batch make.i

make

make install

make clean

9. GEOID Files

a. Obtain the GEOID files from lidar.net.

lidar.net:/mnt/alps/eaarl/tarfiles/geoid03_

pbdfiles.tgz

b. Untar the tarball as a directory in /opt/eaarl/lidar-

processing.

cd /opt/eaarl/lidar-processing

$\operatorname{tar} \mathbf{z x v f} /$ opt/eaarl/tarfiles/geoid03_pbdfiles.tgz 


\section{Appendix B. Virtual Network Computing}

Virtual Network Computing (VNC) is a remote desktop application that allows a connection to a remote computer, as if it were accessed from a keyboard. It consists of two main components: the viewer and the server. The computer to which the user is connected is called the server, and the computer from which the user is connected is the viewer. The viewer is a small application (150 kilobytes for Win32 version) that allows a remote $\mathrm{PC}$ to connect to the server, as well as the ability to view or control the server desktop. The server requires installation of the VNC Service plus the installation of VNC hooks via a registry file. The low-bandwidth requirements permit VNC to run on a wide range of hardware.

To begin a Virtual Network Computing session:

1. Open a secure shell ( $\mathrm{SSH}$ )connection.

2. Create a profile under Profiles menu on the SSH prompt (or click on a previously created profile). Name the profile for the computer that is to be connected.

3. Edit the profile.

a) Click on the Profiles menu.

b) Click Edit Profile....

c) Enter the Host name and User name.

[for example, Host name: computername.er.usgs. gov and User name: jboniste].

d) Message from the server will appear [click OK].

4. Enter the password for computer [click OK].

5. Type in the command line vncserver.

6. The command line will state, "New computername. er.usgs.gov:\# (username) desktop is computername. er.usgs.gov:\#: [for example, New asterix.er.usgs. gov:1 (jboniste) desktop is asterix.er.usgs.gov:1].

7. Open a VNC connection.

8. $\quad$ Enter the specified starting application as computername: \# [for example, asterix:1].

9. Click connect.

10. Enter password. This VNC connection remains unless the connection is somehow killed.

11. When finished, shut down the server. To close a VNC server, type in the SSH prompt: vncserver -kill :\# [for example, vncserver -kill :1]. SSH will state "Killing Xvnc process ID \#\#\#\#" [for example, Killing Xvnc process ID 5065].

12. If the $\mathrm{VNC}$ viewing screen is too large or small, it can be modified by changing the aliases located in the .cshrc file of the user's home directory. The syntax for creating an alias on the command line is:

alias vs vncserver -alwaysshared -depth 16 -geometry

The change will only affect new terminal sessions when vs is typed in the SSH command line.

To reflect in a terminal session that is already open, type this command:

source $/$.cshrc 


\section{Appendix C. Typical EAARL Raw Directory Setup}

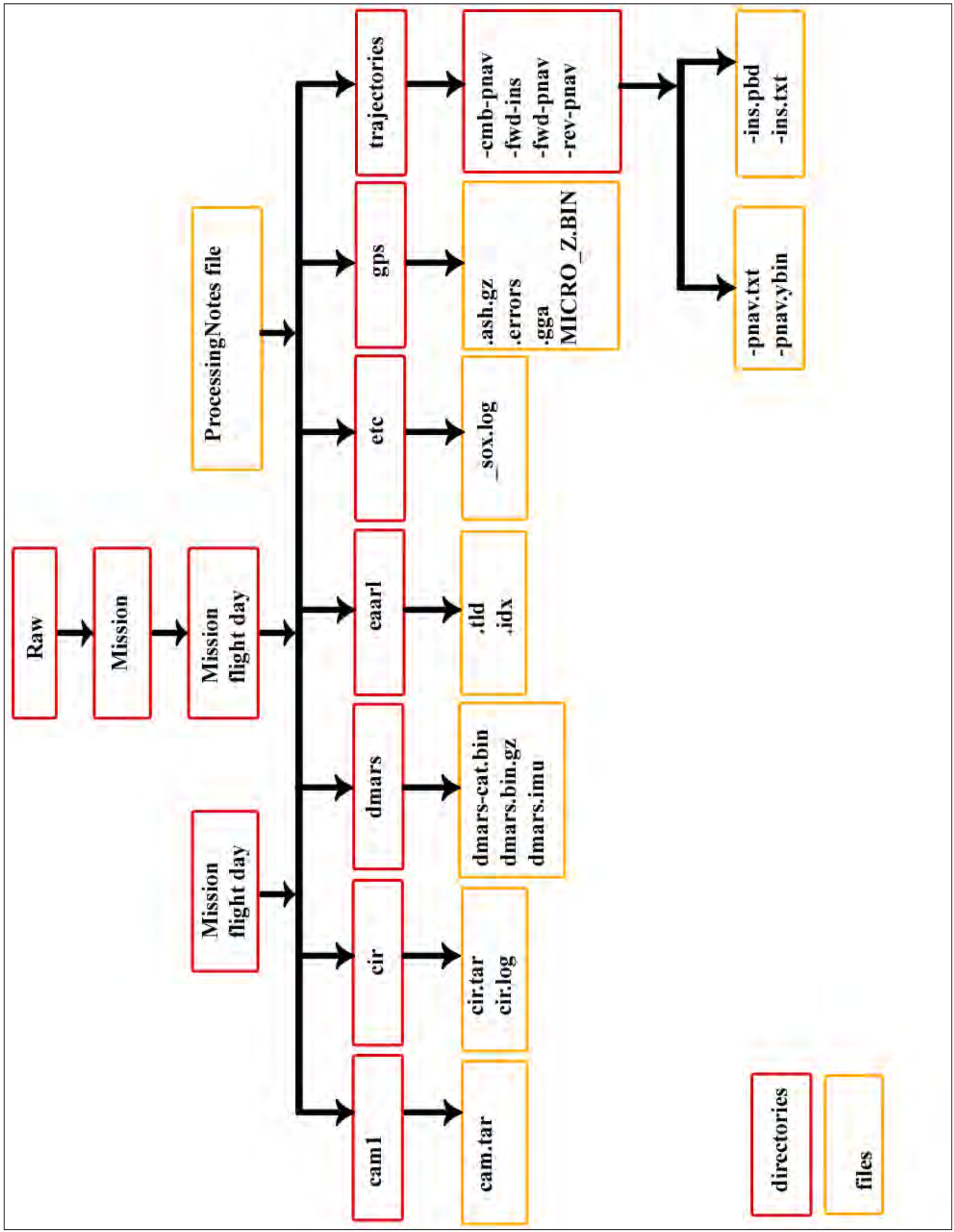




\section{Appendix D. Major EAARL Data Processing Steps}

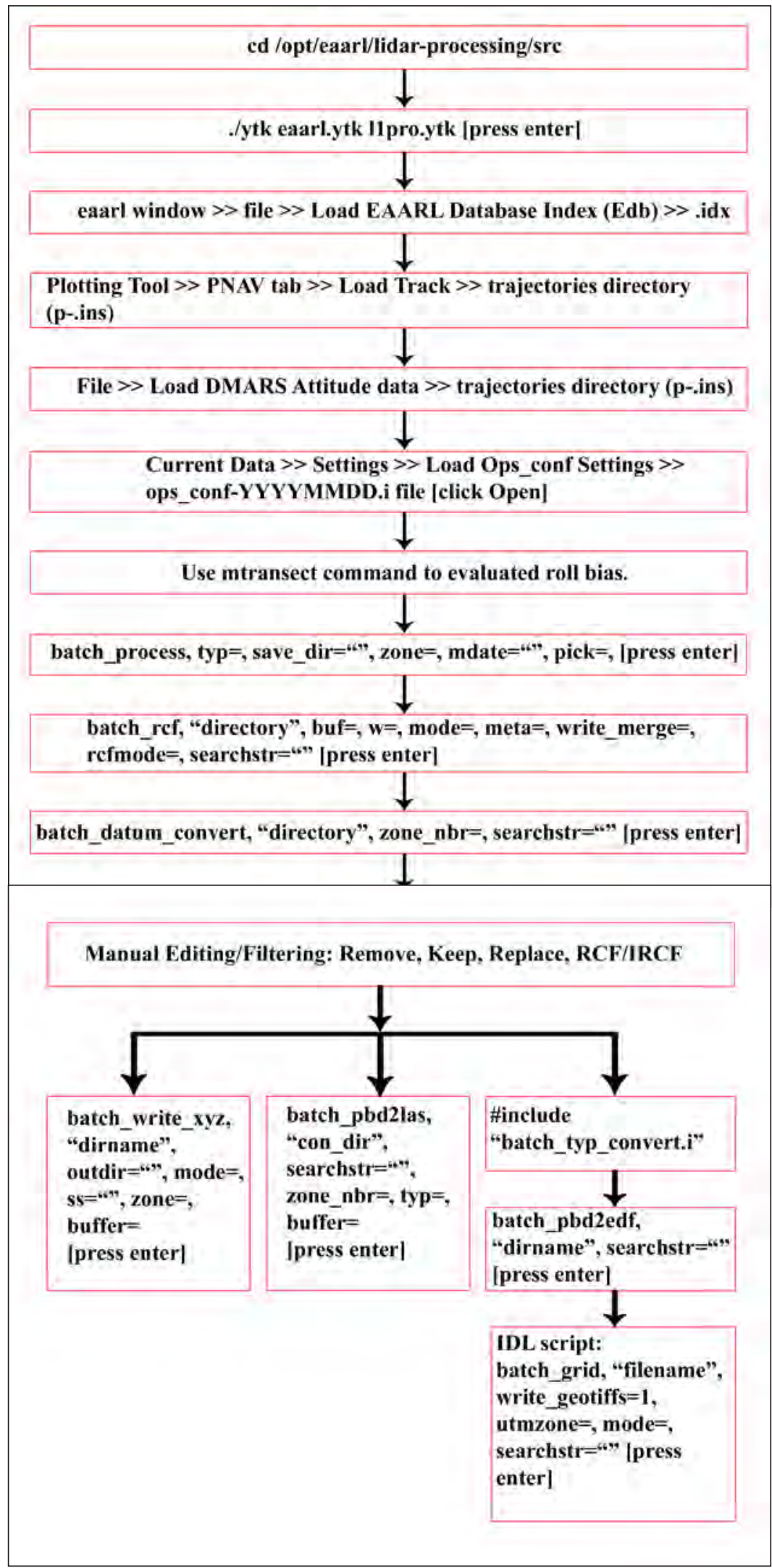




\section{Appendix E: Additional Processing Information}

Other processing modes include:

a) Direct. Wave Spectra displays the dominant wave propagation for the submerged topography data area. The resulting windows appear after the processing has been completed (fig. 35).

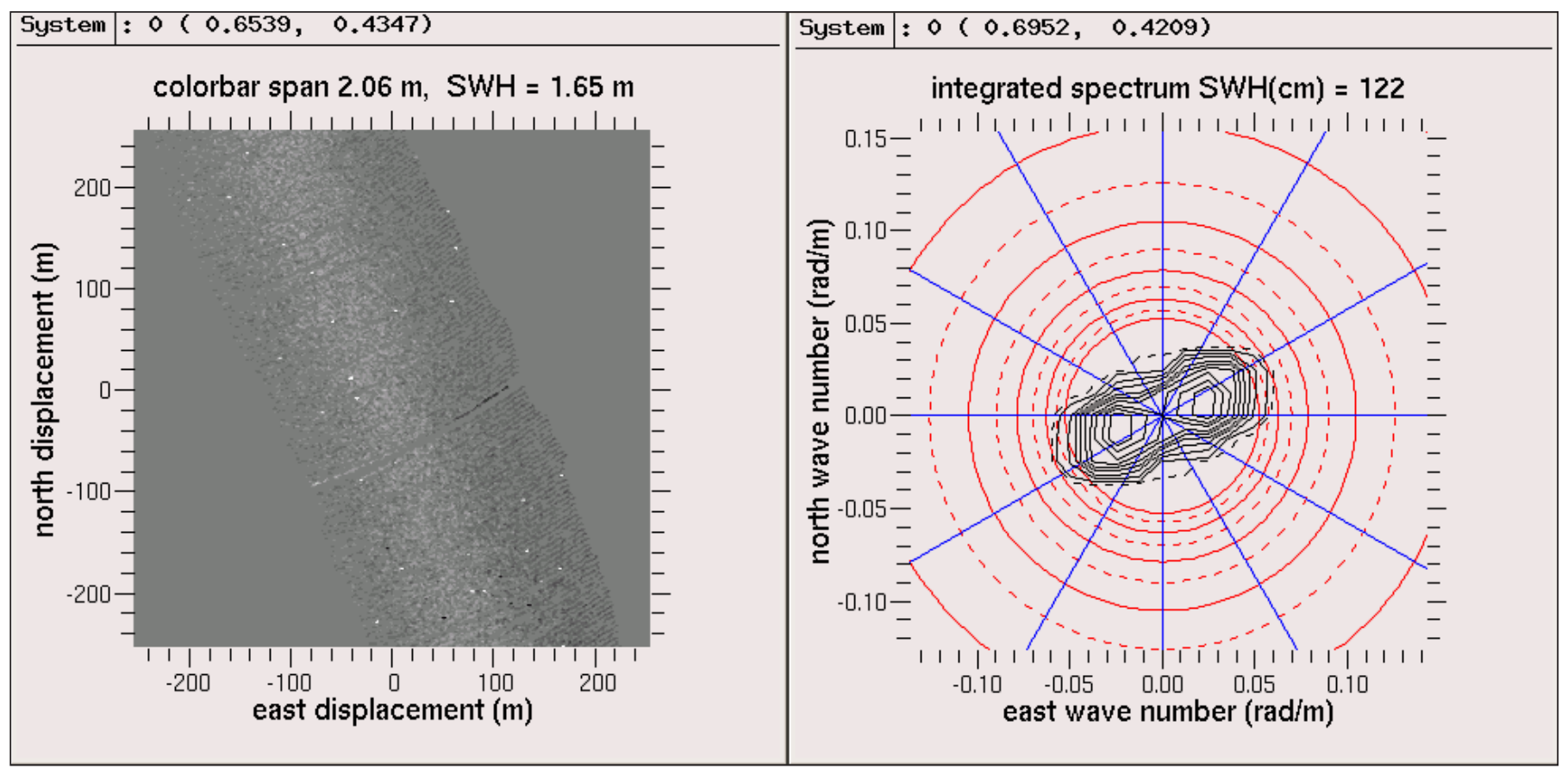

Figure 35. Directional wave spectra display.

b) Multi Peak Veg displays processed discretereturn lidar system data. EAARL is not a discrete system but a waveform-resolving lidar system. This processing mode is not available for evaluating EAARL lidar data. 


\section{List of Abbreviations and Acronyms}

AGL - Above Ground Level

ALPS - Airborne Lidar Processing System

ASCII - American Standard Code for Information Interchange

ASPRS - American Society for Photogrammetry and Remote Sensing

ATM - Airborne Topographic Mapper

CIN - Image Index

CIR - Color-Infrared

CLICK - Center for Lidar Information Coordination and Knowledge

CVS - Concurrent Versions System

DEM - Digital Elevation Model

DMARS - Digital Miniature Attitude Reference System

EAARL - Experimental Advanced Airborne Research Lidar

EDB - EAARL DataBase index file

EDF - EAARL Data Format

GeoTIFF - Georeferenced Tagged Image File Format

GIS - Geographic Information Systems

GPS - Global Positioning System

HMS - Hours Minutes Seconds

IDL - Interactive Data Language

IMU - Inertial Measurement Unit

IRCF - Iterative Random Consensus Filter

JPEG - Joint Photographic Experts Group (Compressed Photographic Images)

KML - Keyhole Markup Language (Google Code)

KMZ - Keyhole Markup Language Zipped (Compressed KML files)

LAS - Log ASCII Standard

lidar - Light Detection And Ranging

NAD83 - North American Datum of 1983

NASA - National Aeronautic and Space Administration

NAVD88 - North American Vertical Datum of 1988
NGS - National Geodetic Survey

NPS - National Park Service

PBD - Processed EAARL Files in Yorick

PDOP - Position Dilution of Precision

PIP - Points In a Polygon

PMT - Photomultiplier Tube

PNAV - Precision Navigation

PNG - Portable Network Graphics

QA - Quality Assurance

QC - Quality Control

QQ - Quarter Quadrangle

RANSAC - Random Sample Consensus

RCF - Random Consensus Filter

RGB - Red-Green-Blue

RMSE - Root Mean Square Error

SOD - Seconds Of the Day

SOW - Seconds Of the Week

SSH - Secure Shell

TIFF - Tagged Image File Format

TIN - Triangulated Irregular Network

USGS - U.S. Geological Survey

UTM - Universal Transverse Mercator

VNC - Virtual Network Computing

WGS84 - World Geodetic System 1984

WGS84 G1150 - World Geodetic System 1984 Precise Ephemeris Beginning GPS Week 1150

YAG - Yttrium Aluminum Garnet 


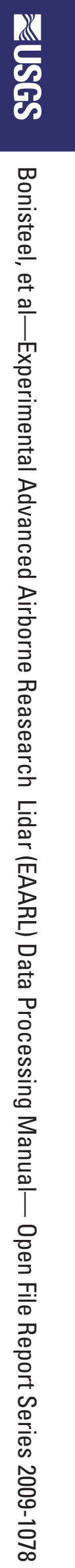